\title{
Quantitative proteomic approach for discriminating major depressive disorder and bipolar disorder by multiple reaction monitoring-mass spectrometry
}

Dongyoon Shin ${ }^{1, \#}$, Sang Jin Rhee ${ }^{3,4, \#, ~ J i h y e o n ~ L e e ~}{ }^{1}$, Injoon Yeo², Misol Do ${ }^{1}$, Eun-Jeong Joo Hee Yeon Jung ${ }^{3,6,7}$, Sungwon Roh ${ }^{8}$, Sang-Hyuk Lee ${ }^{9}$, Hyeyoung Kim ${ }^{10}$, Minji Bang 9 , Kyu Young Lee ${ }^{5}$, Jun Soo Kwon ${ }^{3,4,7}$, Kyooseob $\mathrm{Ha}^{3,4,7}$, Yong Min $\mathrm{Ahn}^{3,4,7, *}$, and Youngsoo Kim ${ }^{1,2, *}$

${ }^{1}$ Department of Biomedical Sciences and ${ }^{2}$ Institute of Medical and Biological Engineering Medical Research Center, Seoul National University College of Medicine, Seoul, Republic of Korea; ${ }^{3}$ Department of Psychiatry, Seoul National University College of Medicine, Seoul, Republic of Korea; ${ }^{4}$ Department of Neuropsychiatry, Seoul National University Hospital, Seoul, Republic of Korea; ${ }^{5}$ Department of Neuropsychiatry, School of Medicine, Eulji University, Daejeon, Republic of Korea; Department of Psychiatry, Nowon Eulji Medical Center, Eulji University, Seoul, Republic of Korea; ${ }^{6}$ Department of Psychiatry, SMG-SNU Boramae Medical Center, Seoul, Republic of Korea; ${ }^{7}$ Institute of Human Behavioral Medicine, Seoul National University Medical Research Center, Seoul, Republic of Korea; ${ }^{8}$ Department of Psychiatry, Hanyang University Hospital, Seoul, Republic of Korea; Department of Psychiatry, Hanyang University College of Medicine, Seoul, Republic of Korea; ${ }^{9}$ Department of Psychiatry, CHA Bundang Medical Center, CHA University School of Medicine, Seongnam, Republic of Korea; ${ }^{10}$ Department of Psychiatry, Inha University Hospital, Incheon, Republic of Korea 


\section{Table of Contents}

Page S-3: Supplementary Method 1. Protein quantification by quantitative targeted proteomics

-Supplementary Method 1.1. Determination of quantifiable targets for MRM-MS analysis

-Supplementary Method 1.2. Plasma sample preparation

-Supplementary Method 1.3. LC-MRM-MS analysis

Page S-8: Supplementary Method 2. MRM-MS data processing

Page S-9: Supplementary Method 3. Model development for discriminating MDD from BD

Page S-11: Abbreviations

Page S-12: References

Page S-16: Supplementary Figure S1. Determination of quantifiable targets for MDD and BD

Page S-17: Supplementary Figure S2. Feature selection and extraction across 100 models generated by repeated application of LASSO regression with ten-fold crossvalidation on the training set

Page S-18: Supplementary Figure S3. AUROC curves representing model performance in discriminating between MDD and BD (without current hypomanic/manic/mixed symptoms), between MDD and BD (drug-free patients), and between patient groups and $\mathrm{HC}$

Page S-19: Supplementary Figure S4. Box-and-whisker plots representing protein abundance of the nine features in all subjects of this study

Page S-20: Supplementary Table S1. The 671 peptides (210 proteins) examined by MRM-MS

Page S-49: Supplementary Table S2. The 210 proteins (210 peptides) selected as candidate features in the training set

Page S-56: Supplementary Table S3. Proportion of features selected for the 210 candidate features (210 proteins/210 peptides) used to determine the model for discriminating MDD from BD

Page S-62: Supplementary Table S4. Summary of unique models originating from combinations of selected features

Page S-64: Supplementary Table S5. Differences in protein abundance of the nine selected features between MDD and BD in the training set

Page S-65: Supplementary Table S6. Differences in protein abundance of the nine selected features between MDD, BD, and HC in the study population (90 MDD, $90 \mathrm{BD}$, and $90 \mathrm{HC}$ )

Page S-67: Supplementary Table S7. Correlations between the nine selected features and demographic/clinical variables in the training set

Page S-69: Supplementary Table S8. Covariate analysis of the four features that showed statistically significant correlation with clinical variables in the training set 


\section{Protein quantification by quantitative targeted proteomics}

\subsection{Determination of quantifiable targets for MRM-MS analysis}

The procedure for selecting integrated protein targets for mood disorders is presented in Supplementary Figure 1. Three types of sources were compiled to generate the list of initial protein targets: 1) proteins that were obtained from our previous study that profiled the proteome of MDD and $\mathrm{BD}(1), 2)$ proteins that originated from previous proteomic studies on mood disorders, including significantly differentially expressed proteins between MDD and $\mathrm{BD}$, between MDD and $\mathrm{HC}$, and between $\mathrm{BD}$ and $\mathrm{HC}(2-16)$, and 3) proteins that have been approved by the US Food and Drug Administration (FDA) and designated as laboratory developed test (LDT). In total, 686 proteins were selected as initial targets after redundant entries were removed.

To investigate targets that had matching MS/MS spectra and unique peptides, 3 MS/MS spectral libraries - the Institute for Systems Biology (https://www.systemsbiology.org), National Institute of Standards and Technology (https://www.nist.gov), and the SWATHAtlas database (www. SWATHAtlas.org)—were used. In total, 648 proteins, corresponding to 7369 unique peptides, were selected.

To examine targets that were detected in blood samples reproducibly, MRM-MS analysis was performed on a pooled plasma sample that consisted of 51 healthy controls (HCs), $40 \mathrm{MDD}$, and $50 \mathrm{BD}$ samples, which did not overlap with individual samples in our current study (90 MDD, 90 BD, and $90 \mathrm{HCs}$ ). Specifically, plasma samples of $3 \mathrm{HCs}$ were collected twice at various time points - once each for the analysis of the pooled sample and the individual samples. Targets were considered detectable if: 1) at least 5 transitions for MRMMS were observed; 2) they had the same elution patterns within the predictive retention time; 
and 3 ) the ratio of transition peaks was obtained as in the spectral library (dot product $>0.8$ ). The dot product score represents the correlation between the peak intensities of transitions that originated from the endogenous peptides of interest and the corresponding entries in the spectral library. Regarding the 648 proteins that corresponded to 7369 unique peptides, 240 MRM-MS methods were generated, and $240 \mathrm{MS}$ runs were performed in total. Reproducibility between MS runs was examined using indexed Retention Time (iRT) standard peptides. The technical reproducibility was evaluated, based on coefficient of variation $(\mathrm{CV})$ values of the intensities and retention time (RT) of iRT peptides - CV value $<15 \%$ for intensity and $<1 \%$ for RT, respectively. Consequently, 412 proteins, corresponding to 1052 unique peptides, were selected as detectable targets in blood.

A total of 1052 SIS peptides, representing 1052 detectable endogenous peptides (412 proteins), were used to examine quantifiable targets. The targets were considered to be quantifiable, based on the following criteria: 1) co-elution of endogenous peptides of interest and corresponding SIS peptides; 2) top 5 peptides per protein, based on rank of peptide intensity; and 3) 1 representative transition per peptide, based on rank of intensity and Automated Detection of Inaccurate and Imprecise Transitions (AuDIT) (17). Consequently, 210 proteins, corresponding to 671 unique peptides and 671 transitions, were determined to be quantifiable targets. These targets were applied to MRM-MS analysis of individual plasma samples of major depressive disorder (MDD) and bipolar disorder (BD) patients and healthy controls (HCs).

\subsection{Plasma sample preparation}

Plasma samples were thawed on ice and centrifuged at $10,000 \mathrm{~g}$ for $10 \mathrm{~min}$ at $4{ }^{\circ} \mathrm{C}$. Supernatants were transferred to fresh tubes and vortexed. For each sample, a volume of 44 
$\mu 1$ was diluted 1:4 with MARS buffer A (Agilent Technologies, Santa Clara, CA, USA) and passed through $0.22 \mu \mathrm{m}$ Spin-X filters (Corning Costar, NY, USA). A volume of $176 \mu$ of buffer A was added to each sample, and each diluted sample was centrifuged through a 0.22 $\mu \mathrm{m}$ filter $(12,000 \mathrm{~g}$, room temperature $)$. Each plasma sample was depleted of 6 highabundance human plasma proteins_-albumin, IgG, IgA, transferrin, haptoglobin, and antitrypsin—using a multiple affinity removal system (MARS) column (Hu-6HC, $4.6 \times 100$ mm, Agilent Technologies, Santa Clara, CA, USA), loaded onto a high-performance liquid chromatography (HPLC) system (Shimadzu Co, Kyoto, Japan). A total of $200 \mu 1$ was injected for each sample. Depleted plasma samples were concentrated by centrifugal filtration for 6 hours at $4^{\circ} \mathrm{C}$ using a 3000-Da molecular weight cutoff (MWCO) filter (Amicon Ultra-4 3K, Millipore, Burlington, MA, USA). Concentrated proteins of plasma samples were quantified by bicinchoninic acid assay (BCA assay) $(18,19)$ using the Pierce ${ }^{\mathrm{TM}}$ BCA Protein Assay Kit (Thermo Scientific, Rockford, IL, USA) (20). BCA assay was performed to measure the concentration of individual samples. A 6-point standard curve was generated by serially diluting an initial concentration of $2 \mathrm{mg} / \mathrm{mL}$ BSA by a factor of 2 . Standards and samples were placed on a 96-well plate, and a mixture of copper solution and BCA solution (1:50) was added. The proteins were digested using RapiGest surfactant and trypsin. A total of $40 \mu 1$ solution of $0.2 \%$ RapiGest, $20 \mathrm{mM}$ dithiothreitol (DTT), and $100 \mathrm{mM}$ ABC buffer, $\mathrm{pH} 8.0$ was added to the $40-\mu l$ plasma samples, adjusted with HPLC-grade water for a $100-\mu \mathrm{g}$ digestion. After 1 hour in $60^{\circ} \mathrm{C}, 20 \mu 100 \mathrm{mM}$ iodoacetamide (IAA) was added. The samples were incubated in the dark for $30 \mathrm{~min}$ at room temperature. Following the incubation, the samples were incubated for 4 hours at $37^{\circ} \mathrm{C}$ after adding trypsin (Sequencing-grade modified, Promega, Madison, WI, USA), dissolved in $50 \mathrm{mM} \mathrm{ABC,} \mathrm{pH} \mathrm{8.0.} \mathrm{Then,} \mathrm{10 \%} \mathrm{formic} \mathrm{acid} \mathrm{was}$ added to the samples to stop the enzymatic reaction (final concentration of formic acid: 1\%), followed by incubation for $30 \mathrm{~min}$ at $37^{\circ}$ to hydrolyze RapiGest surfactant in the acidified 
condition of the samples. After centrifugation at $15,000 \mathrm{rpm}$ at $4{ }^{\circ} \mathrm{C}$ for 1 hour, the precipitation of cleaved RapiGest surfactant was observed. Then, the supernatant without the precipitation was transferred to a new clean tube. The plasma peptide samples (the transferred supernatant for each sample) were spiked with crude stable isotope-labeled internal standard (SIS) peptide, with a C-terminal lysine or arginine heavy-isotope-labeled $\left({ }^{13} \mathrm{C}_{6}{ }^{15} \mathrm{~N}_{2}\right.$ or $\left.{ }^{13} \mathrm{C}_{6}{ }^{15} \mathrm{~N}_{4}\right)$ [purity: crude $(>70 \%)$, JPT, Berlin, Germany]. However, in this study, SIS peptide should have been added as early as possible after the enzymatic reaction in order to correct for any variability in the MS runs as well as any variation associated with sample preparation after the digestion step. The samples were randomly distributed in blocked batches and labeled with identification numbers to blind the researchers throughout the sample preparation.

\subsection{LC-MRM-MS analysis}

In this study, 671 peptides, representing 210 proteins (Supplementary Table 1), were analyzed by targeted multiple reaction monitoring-mass spectrometry (MRM-MS) analysis. MRM-MS analysis was performed on an Agilent 6490 triple quadrupole (QQQ) mass spectrometer (Agilent Technologies, Santa Clara, CA, USA), equipped with a Jetstream electrospray source that was coupled to a 1260 Infinity HPLC system (Agilent Technologies, Santa Clara, CA, USA). Solvents A and B for the HPLC consisted of $0.1 \%$ formic acid/water $(\mathrm{v} / \mathrm{v})$ and $0.1 \%$ formic acid/acetonitrile $(\mathrm{v} / \mathrm{v})$, respectively. Glass vials of the samples in the autosampler were maintained at $4^{\circ} \mathrm{C}$. A total of $40 \mu \mathrm{l}$ of digested sample was injected into a guard column $(2.1 \times 15.0 \mathrm{~mm}, 1.8 \mu \mathrm{m}, 80 \AA)$ (Agilent Technologies, Santa Clara, CA, USA). Online desalting was conducted with the effluent toward waste at $50 \mu \mathrm{l} / \mathrm{min}$ for $10 \mathrm{~min}$ in $3 \%$ solvent B, consisting of $0.1 \%$ formic acid/acetonitrile (v/v), at $40^{\circ} \mathrm{C}$. After the position of valve was switched, the desalted sample was transferred from the guard column to the analytical 
column $(0.5 \times 35.0 \mathrm{~mm}, 3.5 \mu \mathrm{m}, 80 \AA)$ (Agilent Technologies, Santa Clara, CA, USA) in 3\% solvent $\mathrm{B}$, at a flow rate of $40 \mu \mathrm{L} / \mathrm{min}$ for $5 \mathrm{~min}$. The analytical column was heated and maintained at $40^{\circ} \mathrm{C}$ by an oven.

The total run time per LC-MRM-MS analysis was $70 \mathrm{~min}$. Approximately $10 \mu \mathrm{g}$ of digested peptides was injected per LC-MRM-MS run. The peptides were separated on the column and eluted with a linear gradient of $3 \%$ to $35 \%$ acetonitrile $(\mathrm{ACN})$ with $0.1 \%$ formic acid (FA) for $50 \mathrm{~min}$ at $40 \mu \mathrm{L} / \mathrm{min}$. The mass spectra were generated in positive ion mode, based on the following parameters; $2500 \mathrm{~V}$ for the ion spray capillary voltage, $2000 \mathrm{~V}$ for the nozzle voltage, $5 \mathrm{~V}$ for the cell accelerator voltage, $200 \mathrm{~V}$ for the delta $\mathrm{EMV}$, and $380 \mathrm{~V}$ for the fragmented voltage. The drying gas was sprayed at $15 \mathrm{~L} / \mathrm{min}$ at $250^{\circ} \mathrm{C}$, and the sheath gas flow was $12 \mathrm{~L} / \mathrm{min}$ at $350^{\circ} \mathrm{C}$. Collision energy (CE) was optimized by adding the intensities of individual transitions that resulted in the largest peak area. The default value of CE was calculated as follows: $\mathrm{CE}=0.031 \times(\mathrm{m} / \mathrm{z}$ of precursor $)+1$ for double-charged precursor ions and $\mathrm{CE}=0.036 \times(\mathrm{m} / \mathrm{z}$ of precursor $)-4.8$ for triple-charged ions. Five additional steps of adding or subtracting $2 \mathrm{~V}$ on each side of the default value of $\mathrm{CE}$ were predicted.

Five to 10 transition pairs (Q1 and Q3) were selected for each peptides. Subsequently, 1 representative transition for each peptide was determined by rank of intensity of transition and AuDIT. All 671 SIS peptides, representing 210 proteins, were pooled and analyzed to check their retention time (RT). The RTs of the SIS peptides were compared with those of endogenous target peptides by spiking 100 fmol of the pooled mixture of SIS peptides. Subsequently, the final targets - 210 proteins/671 peptides/671 transitions - were quantified in 270 individual blood samples, comprising $90 \mathrm{MDD}, 90 \mathrm{BD}$, and $90 \mathrm{HCs}$. The 270 individual samples were randomly listed in blocked batches with an identification number for each 
sample. Subsequently, the LC-MRM-MS analysis was performed once per sample (1 replicate for each sample). A total of 270 LC-MRM-MS runs were performed.

\section{MRM-MS data processing}

The raw data from the MRM-MS analysis were processed in Skyline, version 19.1.0 (MacCoss Lab, Seattle, WA, USA) to calculate the peak area values of the transitions. Peptidetransition peaks were investigated using peak integrations that were performed manually. Peptide quantification was based on the relative abundance of the endogenous and SIS peptide transitions - ie, the relative abundance of the transition pairs (Q1 and Q3) was determined by the ratio of endogenous (Light) peptide peak areas to the SIS (Heavy) peptide peak areas, reported as peak area ratio (PAR) — Light/Heavy $(\mathrm{L} / \mathrm{H})$ ratio. This process, known as ratio normalization, was used to address any technical variation that occurred across MS runs.

To reduce batch effects that originated from potential technical variabilities, such as the collection of samples across multiple institutions, PAR values were inserted into a webbased public server (https://genepattern.broadinstitute.org). Batch correction with the Combat algorithm — a nonparametric adjustment for reducing batch effects using an empirical Bayes framework —was performed. Subsequently, the PAR values were log2-scaled.

Then, through the R package caret (21), the data were randomly distributed at a ratio of 8:2 to designate the training set for model development and the test set for evaluation of the model's performance.

\section{Model development for discriminating MDD from BD}


Model averaging, based on the Akaike's information criterion (AIC), was implemented. The AIC measures relatively how well a model approximates the given data, wherein the model with the lowest AIC value is considered to be the best model (22-25). The bias-corrected version of AIC (AICc) was adopted, because the sample size (n) was small compared with the largest value of $\mathrm{k}$, where $\mathrm{k}$ is the number of features that was selected in a model (22-25). AICc was used to calculate the Akaike weight (w) of each model, deemed as the probability when one model was the best approximating model for the data (22-25). The Akaike weight had a value between 0 and 1, and the sum of weights of all models was equal to 1. With respect to each unique model, the weight values of all corresponding models were summed to estimate the probability that the combination of selected features constituted the best approximating model. The average coefficient of a given feature across a set of 100 models, $\hat{\bar{B}}$, was calculated as follows: $\hat{\bar{B}}=\sum_{m=1}^{100} w_{m} \hat{B}_{m}$, where $w_{m}$ and $\hat{B}_{m}$ represent the Akaike weight and the estimated coefficient of a feature in model $\mathrm{m}$, respectively. Features that were never selected were not able to contribute to the estimation of the average coefficient, because the coefficient converges to 0 .

Specifically, the general formula of the AIC is as follows: $A I C=-2 \ln (L)+2 k$, where $L$ represents the likelihood function and $k$ represents the number of features that is selected in a model $(13,14)$. In this study, the bias-corrected version of AIC was considered. The $\mathrm{AIC}_{\mathrm{c}}$ is as follows: $A I C_{c}=A I C+\frac{2 k(k+1)}{n-k-1}$, where $n$ represents sample size and $k$ represents the number of features that is selected in a model $(15,16)$. With regard to a given model $\mathrm{m}$ in a set of 100 models, the Akaike weight, $w_{m}$, was computed using the difference between its $\mathrm{AIC}_{c}$ value and the lowest $\operatorname{AIC}_{c}$ value $(24,26)$. The relevant formulas are as follows: $\Delta_{m}=A I C_{c m}-A I C_{c \min }$ and $w_{m}=\frac{\exp \left(-\frac{1}{2} \Delta_{m}\right)}{\sum_{m=1}^{100} \exp \left(-\frac{1}{2} \Delta_{m}\right)}$. 
The methods and formulas for model development in this study referred to those of previous studies (27-29). 


\begin{abstract}
ABBREVIATIONS
MDD, major depressive disorder; $\mathrm{BD}$, bipolar disorder; MRM, multiple reaction monitoring; MS, mass spectrometry; HC, healthy control; FDA, US Food and Drug Administration; LDT, laboratory developed test; iRT, indexed Retention Time; RT, retention time; $\mathrm{ACN}$, acetonitrile; FA, formic acid; $\mathrm{CE}$, collision energy; $\mathrm{CV}$, coefficient of variation; HPLC, high-performance liquid chromatography; MWCO, molecular weight cutoff; BCA, bicinchoninic acid; DTT, dithiothreitol; IAA, iodoacetamide; SIS, stable isotope-labeled internal standard; QQQ, triple quadrupole; PAR, peak area ratio; L/H, Light/Heavy; AIC, Akaike's information criterion; AICc, the bias-corrected version of AIC; w, Akaike weight; AuDIT, automated detection of inaccurate and imprecise transitions
\end{abstract}




\section{REFERENCES}

1. Rhee SJ, Han D, Lee Y, Kim H, Lee J, Lee K, et al. Comparison of serum protein profiles between major depressive disorder and bipolar disorder. BMC Psychiatry 2020, 20(1):145.

2. Comes AL, Papiol S, Mueller T, Geyer PE, Mann M, Schulze TG. Proteomics for blood biomarker exploration of severe mental illness: pitfalls of the past and potential for the future. Transl Psychiatry 2018, 8(1):160.

3. Alsaif, M. et al. Analysis of serum and plasma identifies differences in molecular coverage, measurement variability, and candidate biomarker selection. Proteom. Clin. Appl. 2012, 6, 297-303.

4. Bot, M. et al. Serum proteomic profiling of major depressive disorder. Transl. Psychiatry 2015, 5, e599.

5. Frye, M. A. et al. Feasibility of investigating differential proteomic expression in depression: implications for biomarker development in mood disorders. Transl. Psychiatry 2015, 5, e689.

6. Haenisch, F. et al. Multiplex immunoassay analysis of plasma shows prominent upregulation of growth factor activity pathways linked to GSK3beta signaling in bipolar patients. J. Affect Disord. 2014, 156, 139-143.

7. Herberth, M. et al. Peripheral profiling analysis for bipolar disorder reveals markers associated with reduced cell survival. Proteomics 2011, 11, 94-105

8. Lee, M. Y. et al. Discovery of serum protein biomarkers in drug-free patients with major depressive disorder. Prog. Neuropsychopharmacol. Biol. Psychiatry 2016, 69, $60-68$. 
9. Stelzhammer, V. et al. Proteomic changes in serum of first onset, antidepressant drugnaïve major depression patients. Int. J. Neuropsychopharmacol. 2014, 17, 15991608.

10. $\mathrm{Xu}, \mathrm{H}$.-B. et al. Comparative proteomic analysis of plasma from major depressive patients: Identification of proteins associated with lipid metabolism and immunoregulation. Int. J. Neuropsychopharmacol. 2012, 15, 1413-1425.

11. Yuan N, Chen Y, Xia Y, Dai J, Liu C. Inflammation-related biomarkers in major psychiatric disorders: a cross-disorder assessment of reproducibility and specificity in 43 meta-analyses. Transl Psychiatry 2019, 9(1):233.

12. Lee MY, Kim EY, Kim SH, Cho KC, Ha K, Kim KP, et al. Discovery of serum protein biomarkers in drug-free patients with major depressive disorder. Prog Neuropsychopharmacol Biol Psychiatry 2016, 69: 60-68.

13. Chen J, Huang C, Song Y, Shi H, Wu D, Yang Y, et al. Comparative proteomic analysis of plasma from bipolar depression and depressive disorder: identification of proteins associated with immune regulatory. Protein Cell 2015, 6(12): 908-911.

14. Ren J, Zhao G, Sun X, Liu H, Jiang P, Chen J, et al. Identification of plasma biomarkers for distinguishing bipolar depression from major depressive disorder by iTRAQ-coupled LC-MS/MS and bioinformatics analysis. Psychoneuroendocrinology 2017, 86: 17-24.

15. Rhee SJ, Han D, Lee Y, Kim H, Lee J, Lee K, et al. Comparison of serum protein profiles between major depressive disorder and bipolar disorder. BMC Psychiatry 2020, 20(1): 145 .

16. Kittel-Schneider S, Hahn T, Haenisch F, McNeill R, Reif A, Bahn S. Proteomic Profiling as a Diagnostic Biomarker for Discriminating Between Bipolar and 
Unipolar Depression. Frontiers in psychiatry 2020, 11: 189.

17. Abbatiello SE, Mani DR, Keshishian H, Carr SA. Automated detection of inaccurate and imprecise transitions in peptide quantification by multiple reaction monitoring mass spectrometry. Clin Chem. 2010,56(2):291-305.

18. Walker JM. The bicinchoninic acid (BCA) assay for protein quantitation. Methods Mol Biol 1994; 32: 5-8.

19. Hill HD, Straka JG. Protein determination using bicinchoninic acid in the presence of sulfhydryl reagents. Anal Biochem 1988; 170(1): 203-208.

20. Schretter CE, Vielmetter J, Bartos I, Marka Z, Marka S, Argade S, et al. A gut microbial factor modulates locomotor behaviour in Drosophila. Nature 2018; 563(7731): 402-406.

21. Kuhn M. Building Predictive Models in R Using the caret Package. 2008 2008; 28(5): $26 \% \mathrm{~J}$ Journal of Statistical Software.

22. Burnham, K. P. \& Anderson, D. R. In Model Selection and Multi-Model Inference: A Practical Information-Theoretical Approach, second edn. Springer, 2002.

23. Akaike, H. Information theory and an extension of the maximum likelihood principle. In Second International Symposium on Information Theory (eds Petrov, B. N. \& Csaki, F.) 267-281 (Budapest, Akadémiai Kiado0), 1973.

24. Hurvich, C. M. \& Tsai, C. L. Regression and time series model selection in small samples. Biometrika 1989, 76, 297-307.

25. Sugiura, N. Further analysis of the data by akaike's information criterion and the finite corrections. Commun. Stat. - Theory Methods 1978, 27, 13-26.

26. Burnham, K. P. \& Anderson, D. R. Multimodel inference: understanding AIC and BIC in model selection. Socio. Methods Res 2004, 33, 261-304 (2004).

27. Han SYS, Cooper JD, Ozcan S, Rustogi N, Penninx B, Bahn S. Integrating proteomic, 
sociodemographic and clinical data to predict future depression diagnosis in subthreshold symptomatic individuals. Transl Psychiatry. 2019, 9(1):277.

28. Cooper JD, Han SYS, Tomasik J, Ozcan S, Rustogi N, van Beveren NJM, et al. Multimodel inference for biomarker development: an application to schizophrenia. Transl Psychiatry. 2019, 9(1):83.

29. Han SYS, Tomasik J, Rustogi N, Lago SG, Barton-Owen G, Eljasz P, et al. Diagnostic prediction model development using data from dried blood spot proteomics and a digital mental health assessment to identify major depressive disorder among individuals presenting with low mood. Brain Behav Immun 2020, 90: 184-195. 


\section{Supplementary Figure S1. Determination of quantifiable targets for MDD and BD.}

Overall process, including integration of initial protein targets, selection of targets by public spectrum libraries, selection of MS detectable targets, and determination of final quantifiable targets. The number of selected targets for each step is represented. The final quantifiable targets were applied to MRM-MS analysis of individual blood samples of MDD patients, BD patients, and healthy controls. MS, mass spectrometry; MRM, multiple reaction monitoring; MDD, major depressive disorder; BD, bipolar disorder; HC, healthy control; FDA, US Food and Drug Administration; LDT, laboratory developed test; AuDIT, automated detection of inaccurate and imprecise transitions

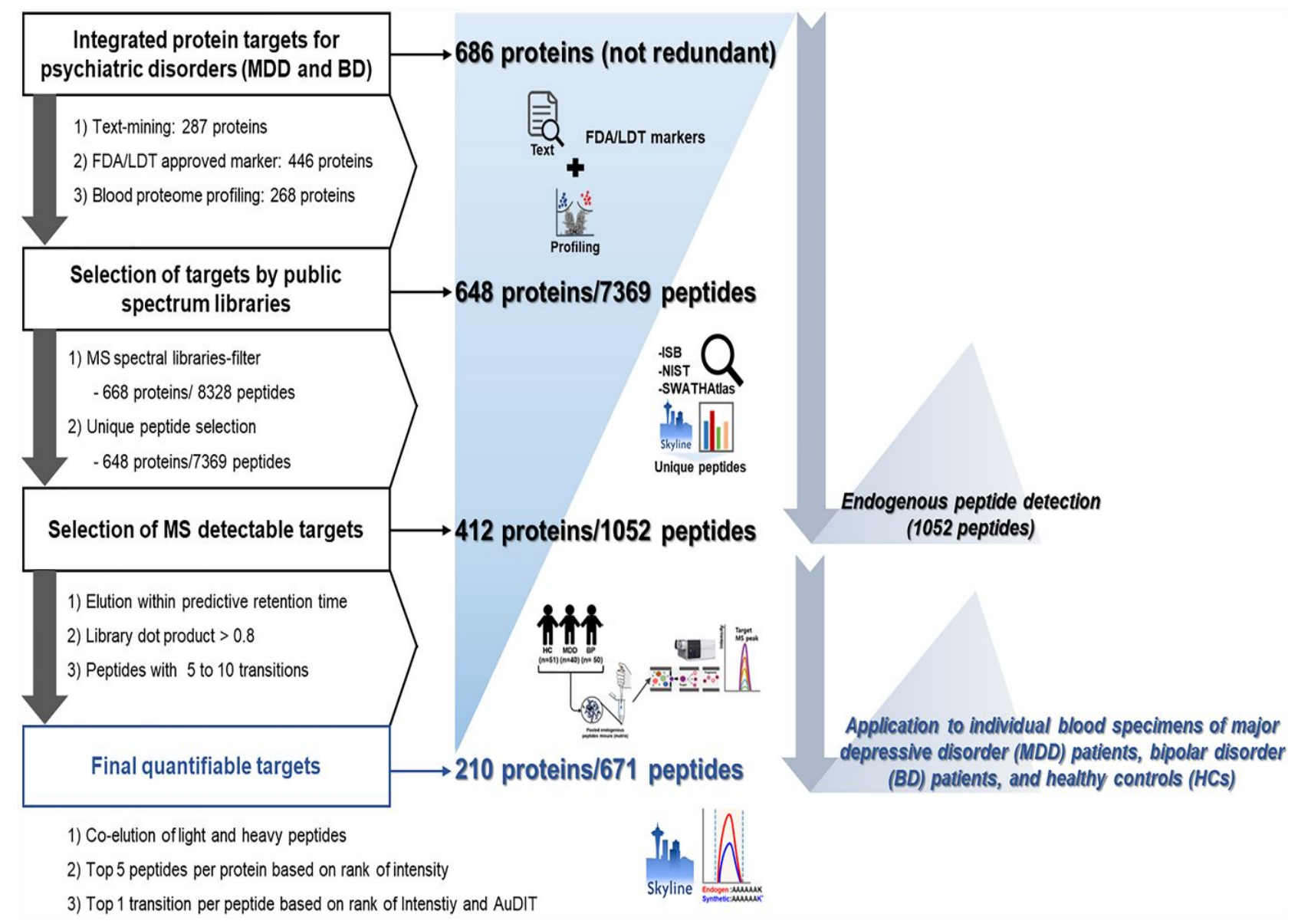


Supplementary Figure S2. Feature selection and extraction across 100 models generated by repeated application of LASSO regression with ten-fold crossvalidation on the training set. A. Frequency of selected features for the 100 obtained models. Perturbed distribution between model frequency and number of selected features was showed. B. Proportion of features selected with respect to the 210 candidate features. A total of 26 features among 210 candidate features had more than 0 of the value of proportion of feature selected. Total 9 features had more than 0.9 of the value of proportion of feature selected value.

A

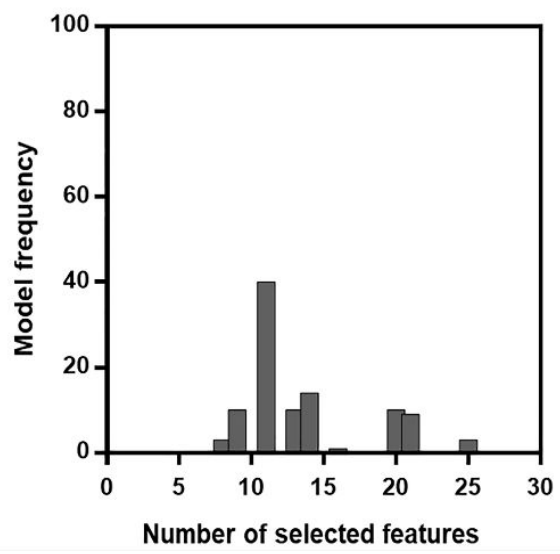

B

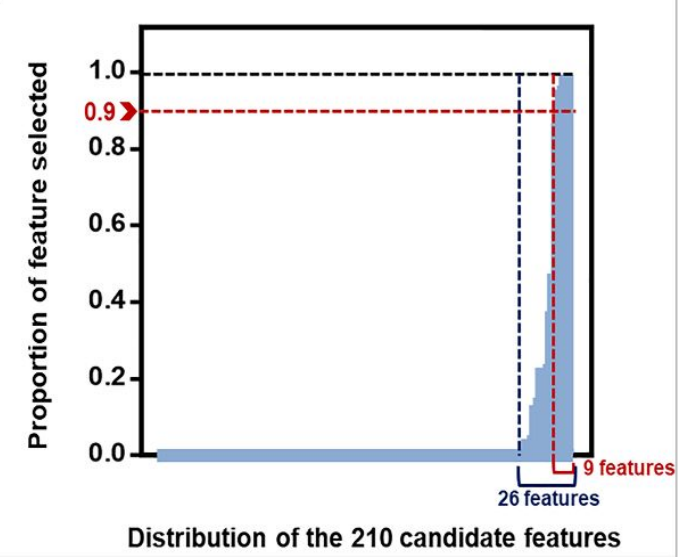


Figure S3. AUROC curves representing model performance in discriminating between MDD and BD (without current hypomanic/manic/mixed symptoms), between MDD and BD (drug-free patients), and between patient groups and HC. The developed model for discriminating MDD from $\mathrm{BD}$ was applied to data on patient groups and $\mathrm{HCs}$ in all study subjects. A. The model's performance in the patient groups (90 MDD patients vs 75 BD patients without current hypomanic/manic/mixed symptoms). B. The model's performance in drug-free MDD and BD patients (11 MDD vs $10 \mathrm{BD})$. C. The model's performance in the MDD group and HC (90 MDD patients vs $90 \mathrm{HCs}$ ). D. The model's performance in the BD group and $\mathrm{HC}(90 \mathrm{BD}$ patients vs $90 \mathrm{HCs})$. AUC, area under the curve; MDD, major depressive disorder; $\mathrm{BD}$, bipolar disorder; $\mathrm{HC}$, healthy control; AUROC, area under receiver operating characteristics
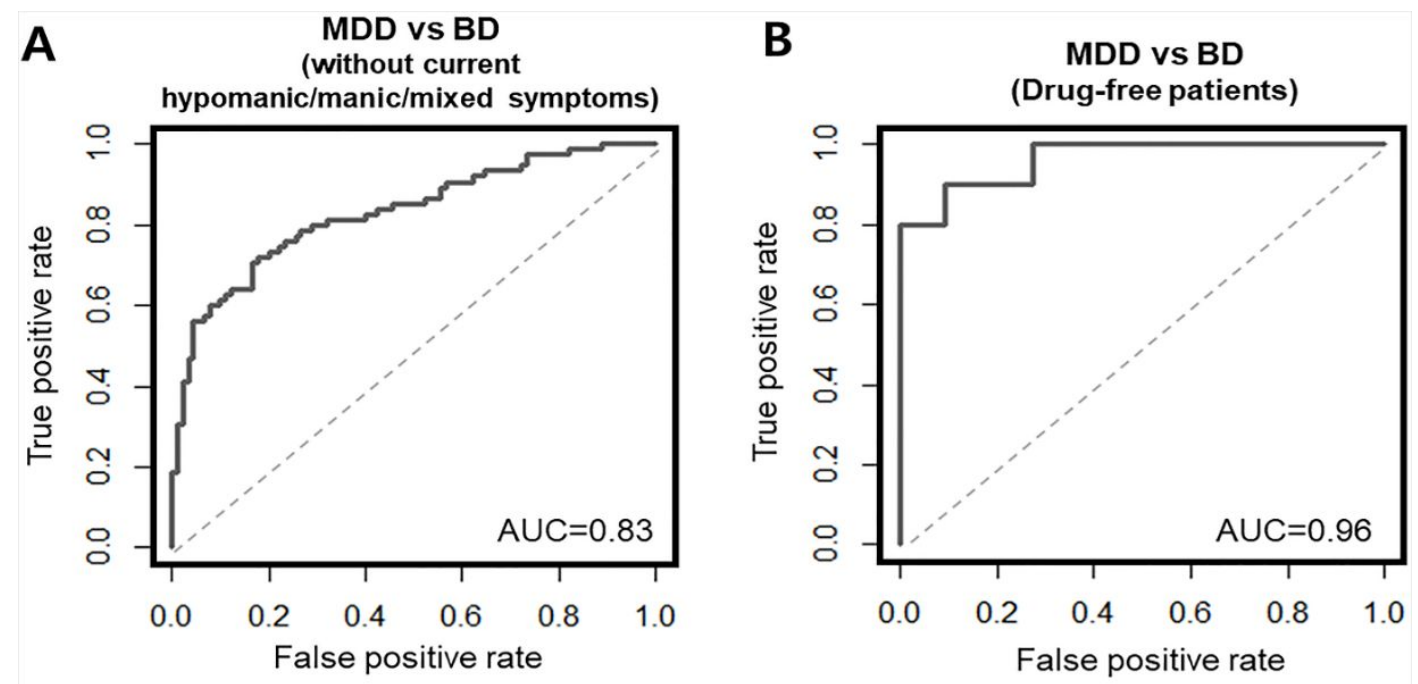

C
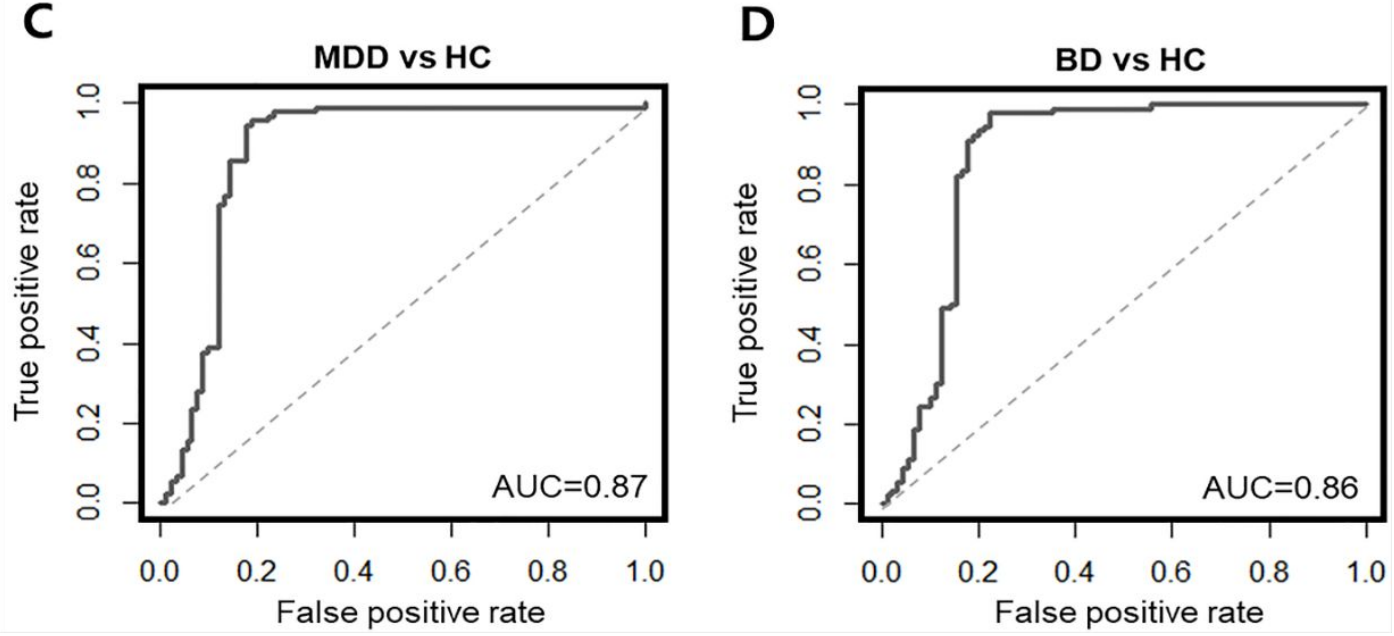
Figure S4. Box-and-whisker plots representing protein abundance of the nine features in all subjects of this study. The distributions of protein abundance between the MDD, BD, and HC groups are shown in all study subjects. Protein abundance is represented by the $\log _{2}-$ transformed peak area ratio (Light/Heavy ratio). One-way ANOVA (for three groups) was performed to examine statistically significant differences between the 3 groups. Subsequently, post hoc analysis was performed by Tukey's HSD. L/H ratio, Light/Heavy ratio; HC, healthy control; MDD, major depressive disorder; and BD, bipolar disorder; HSD, honestly significant difference; ANOVA, analysis of variance; *, $P<0.05$; **, $P<0.01$; ***, $P<0.001$; ****, $P<0.0005$; n.s., no significance
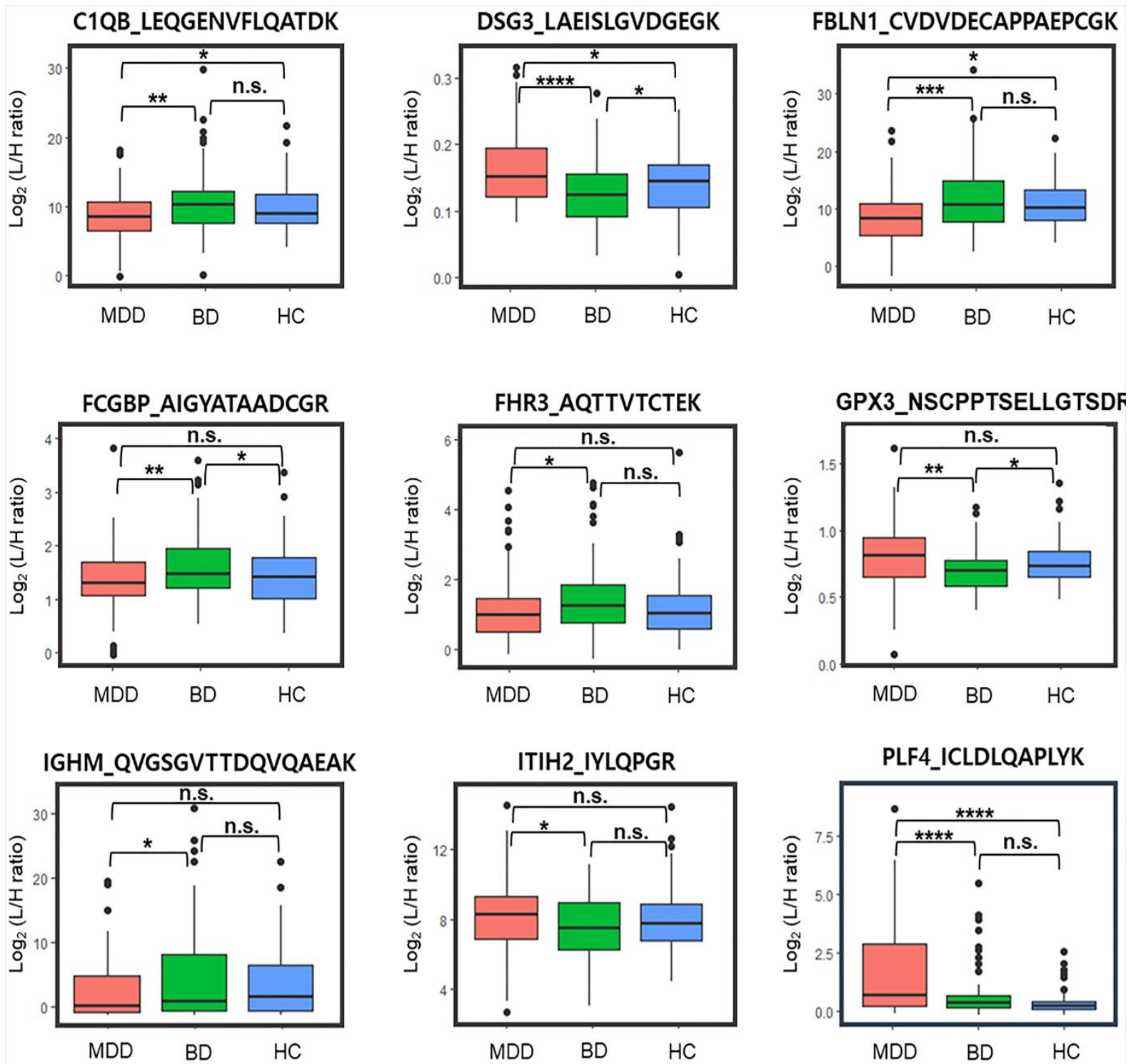
Supplementary Table S1. The 671 peptides (210 proteins) examined by MRM-MS ${ }^{a}$

\begin{tabular}{|c|c|c|c|c|c|c|c|c|c|c|c|c|c|c|}
\hline \multicolumn{3}{|c|}{ The final quantifiable targets information } & \multicolumn{12}{|c|}{ Mass Information } \\
\hline $\begin{array}{l}\text { Uniprot } \\
\text { accessio } \\
\text { n number }\end{array}$ & Protein & Peptide & $\begin{array}{l}\text { Precursor } \\
\text { ion. Light } \\
(\mathrm{m} / \mathrm{z})\end{array}$ & $\begin{array}{c}\text { Precursor } \\
\text { ion. Heavy } \\
(\mathrm{m} / \mathrm{z})\end{array}$ & $\begin{array}{l}\text { Precur } \\
\text { sor ion } \\
\text { charge }\end{array}$ & $\begin{array}{l}\text { Product } \\
\text { ion. Light } \\
(\mathrm{m} / \mathrm{z})\end{array}$ & $\begin{array}{c}\text { Additional } \\
\text { product } \\
\text { ion.Light } \\
(\mathrm{m} / \mathrm{z})^{\mathrm{b}}\end{array}$ & $\begin{array}{l}\text { Produ } \\
\text { ct ion. } \\
\text { Heavy } \\
(\mathrm{m} / \mathrm{z})\end{array}$ & $\begin{array}{l}\text { Produc } \\
t \text { ion } \\
\text { charge }\end{array}$ & $\begin{array}{c}\text { Additional } \\
\text { product } \\
\text { ion } \\
\text { charge }^{b}\end{array}$ & $\begin{array}{l}\text { Produ } \\
\text { ct ion } \\
\text { type }\end{array}$ & $\begin{array}{l}\text { Additiona } \\
\text { I product } \\
\text { ion type }^{\text {b }}\end{array}$ & $\begin{array}{c}\text { Product } \\
\text { ion } \\
\text { Quantifie } \\
r^{c}\end{array}$ & $\begin{array}{c}\text { Collision } \\
\text { energy } \\
\text { (volt) }\end{array}$ \\
\hline P02763 & A1AG1 & EQLGEFYEALDCLR & 871.9 & 876.9 & 2 & 258.1 & 876.4 & 258.1 & 1 & 1 & b2 & y7 & Y & 28 \\
\hline P02763 & A1AG1 & SDVVYTDWK & 556.8 & 560.8 & 2 & 712.3 & & 720.3 & 1 & & y5 & & Y & 18.3 \\
\hline P19652 & A1AG2 & EQLGEFYEALDCLCIPR & 1057 & 1062 & 2 & 272.2 & 933.4 & 282.2 & 1 & 1 & y2 & y7 & Y & 33.8 \\
\hline P04217 & A1BG & ATWSGAVLAGR & 544.8 & 549.8 & 2 & 730.4 & & 740.4 & 1 & & y8 & & Y & 17.9 \\
\hline P04217 & A1BG & CEGPIPDVTFELLR & 823.4 & 828.4 & 2 & 1089.6 & & 1099.6 & 1 & & y9 & & Y & 26.5 \\
\hline P04217 & A1BG & ELLVPR & 363.7 & 368.7 & 2 & 272.2 & 484.3 & 282.2 & 1 & 1 & y2 & y4 & Y & 12.3 \\
\hline P04217 & $\mathrm{A} 1 \mathrm{BG}$ & LLELTGPK & 435.8 & 439.8 & 2 & 644.4 & & 652.4 & 1 & & y6 & & Y & 14.5 \\
\hline P04217 & A1BG & VTLTCVAPLSGVDFQLR & 938.5 & 943.5 & 2 & 1131.6 & & 1141.6 & 1 & & $\mathrm{y} 10$ & & $\mathrm{Y}$ & 30.1 \\
\hline P08697 & $\mathrm{A} 2 \mathrm{AP}$ & DFLQSLK & 425.7 & 429.7 & 2 & 588.4 & & 596.4 & 1 & & y5 & & Y & 14.2 \\
\hline P08697 & $\mathrm{A} 2 \mathrm{AP}$ & LCQDLGPGAFR & 617.3 & 622.3 & 2 & 547.3 & & 557.3 & 1 & & y5 & & Y & 20.1 \\
\hline P08697 & $\mathrm{A} 2 \mathrm{AP}$ & LFGPDLK & 395.2 & 399.2 & 2 & 529.3 & & 537.3 & 1 & & y5 & & Y & 13.3 \\
\hline P08697 & $\mathrm{A} 2 \mathrm{AP}$ & QEDDLANINQWVK & 786.9 & 790.9 & 2 & 674.4 & & 682.4 & 1 & & y5 & & $\mathrm{Y}$ & 25.4 \\
\hline P01023 & A2MG & ALLAYAFALAGNQDK & 783.4 & 787.4 & 2 & 561.3 & & 569.3 & 1 & & y5 & & Y & 25.3 \\
\hline P01023 & A2MG & EQAPHCICANGR & 471.5 & 474.9 & 3 & 494.2 & & 499.2 & 2 & & y8 & & Y & 12.2 \\
\hline P01023 & A2MG & HNVYINGITYTPVSSTNEK & 1069 & 1073 & 2 & 861.4 & & 869.4 & 1 & & y8 & & Y & 34.1 \\
\hline P01023 & A2MG & NEDSLVFVQTDK & 697.8 & 701.9 & 2 & 737.4 & & 745.4 & 1 & & y6 & & Y & 22.6 \\
\hline P01023 & A2MG & QGIPFFGQVR & 574.8 & 579.8 & 2 & 850.5 & & 860.5 & 1 & & y7 & & $\mathrm{Y}$ & 18.8 \\
\hline P01023 & A2MG & TEVSSNHVLIYLDK & 809.4 & 813.4 & 2 & 231.1 & 538.3 & 231.1 & 1 & 1 & b2 & y4 & $\mathrm{Y}$ & 26.1 \\
\hline P01023 & A2MG & VTAAPQSVCALR & 424.9 & 428.2 & 3 & 519.3 & & 529.3 & 1 & & y4 & & Y & 10.5 \\
\hline
\end{tabular}




\begin{tabular}{|l|l|l|} 
P01023 & A2MG & VYDYYETDEFAIAEYNAPCSK \\
\hline Q15848 & ADIPO & GDIGETGVPGAEGPR \\
\hline P43652 & AFAM & FTFEYSR \\
\hline P43652 & AFAM & HFQNLGK \\
\hline P43652 & AFAM & IAPQLSTEELVSLGEK \\
\hline P43652 & AFAM & TNFAFR \\
\hline P43652 & AFAM & YHYLIR \\
\hline P02768 & ALBU & LVNEVTEFAK \\
\hline P04075 & ALDOA & ALQASALK \\
\hline P35858 & ALS & ANVFVQLPR \\
\hline P35858 & ALS & DFALQNPSAVPR \\
\hline P35858 & ALS & LAELPADALGPLQR \\
\hline P35858 & ALS & LEALPNSLLAPLGR \\
\hline P35858 & ALS & LHSLHLEGSCLGR \\
\hline P02760 & AMBP & CVLFPYGGCQGNGNK \\
\hline P02760 & AMBP & ECLQTCR \\
\hline P02760 & AMBP & ETLLQDFR \\
\hline P02760 & AMBP & GVCEETSGAYEK \\
\hline P02760 & AMBP & TVAACNLPIVR \\
\hline P15144 & AMPN & AQIINDAFNLASAHK \\
\hline P54802 & ANAG & DFCGCHVAWSGSQLR \\
\hline P01019 & ANGT & DPTFIPAPIQAK \\
\hline P01019 & ANGT & LQAILGVPWK \\
\hline P01019 & ANGT & QPFVQGLALYTPVVLPR \\
\hline
\end{tabular}

$\begin{array}{llll}1274.5 & 1278.6 & 2 & 491.2 \\ 706.3 & 711.3 & 2 & 839.4 \\ 475.2 & 480.2 & 2 & 701.3 \\ 422.2 & 426.2 & 2 & 204.1 \\ 572 & 574.7 & 3 & 632.4 \\ 378.2 & 383.2 & 2 & 540.3 \\ 432.7 & 437.7 & 2 & 351.2 \\ 575.3 & 579.3 & 2 & 937.5 \\ 401.2 & 405.3 & 2 & 157.1 \\ 522.3 & 527.3 & 2 & 759.5 \\ 657.8 & 662.8 & 2 & 626.4 \\ 732.4 & 737.4 & 2 & 314.2 \\ 732.4 & 737.4 & 2 & 1037.6 \\ 493.6 & 496.9 & 3 & 614.8 \\ 835.9 & 839.9 & 2 & 260.1 \\ 483.7 & 488.7 & 2 & 436.2 \\ 511.3 & 516.3 & 2 & 565.3 \\ 665.3 & 669.3 & 2 & 884.4 \\ 607.3 & 612.3 & 2 & 484.3 \\ 538.3 & 541 & 3 & 200.1 \\ 593.9 & 597.3 & 3 & 759.3 \\ 649.4 & 653.4 & 2 & 724.4 \\ 562.8 & 566.9 & 2 & 883.5 \\ 633.4 & 636.7 & 3 & 680.4\end{array}$

\begin{tabular}{|c|c|c|}
\hline & 499.2 & 1 \\
\hline & 849.4 & 1 \\
\hline & 711.3 & 1 \\
\hline \multirow[t]{11}{*}{527.2} & 212.1 & 1 \\
\hline & 640.4 & 1 \\
\hline & 550.3 & 1 \\
\hline & 356.2 & 2 \\
\hline & 945.5 & 1 \\
\hline & 157.1 & 2 \\
\hline & 769.5 & 1 \\
\hline & 636.4 & 1 \\
\hline & 314.2 & 1 \\
\hline & 1047.6 & 1 \\
\hline & 619.8 & 2 \\
\hline \multirow[t]{5}{*}{373.2} & 260.1 & 1 \\
\hline & 446.2 & 1 \\
\hline & 575.3 & 1 \\
\hline & 892.4 & 1 \\
\hline & 494.3 & 1 \\
\hline \multirow[t]{5}{*}{313.7} & 200.1 & 1 \\
\hline & 764.3 & 2 \\
\hline & 732.4 & 1 \\
\hline & 891.6 & 1 \\
\hline & 690.5 & 1 \\
\hline
\end{tabular}

\begin{tabular}{|c|c|c|c|}
\hline y4 & & $Y$ & 40.5 \\
\hline y9 & & $Y$ & 22.9 \\
\hline y5 & & $Y$ & 15.7 \\
\hline y2 & b4 & $Y$ & 14.1 \\
\hline y6 & & $Y$ & 15.8 \\
\hline y4 & & $Y$ & 12.7 \\
\hline y5 & & $Y$ & 14.4 \\
\hline y8 & & $Y$ & 18.8 \\
\hline b3 & & $Y$ & 13.4 \\
\hline y6 & & $Y$ & 17.2 \\
\hline y6 & & $Y$ & 21.4 \\
\hline b3 & & $Y$ & 23.7 \\
\hline y10 & & $Y$ & 23.7 \\
\hline y11 & & $Y$ & 13 \\
\hline b2 & b3 & $Y$ & 26.9 \\
\hline y3 & & $Y$ & 16 \\
\hline y4 & & $Y$ & 16.8 \\
\hline y8 & & $Y$ & 21.6 \\
\hline y4 & & $Y$ & 19.8 \\
\hline b2 & y6 & $Y$ & 14.6 \\
\hline y13 & & $Y$ & 16.6 \\
\hline y7 & & $Y$ & 21.1 \\
\hline y8 & & $Y$ & 18.4 \\
\hline y6 & & $Y$ & 18 \\
\hline
\end{tabular}




\begin{tabular}{|l|l|l|} 
P01019 & ANGT & SLDFTELDVAAEK \\
\hline P01019 & ANGT & TSPVDEK \\
\hline P01019 & ANGT & VLSALQAVQGLLVAQGR \\
\hline P01008 & ANT3 & ENAEQSR \\
\hline P01008 & ANT3 & FATTFYQHLADSK \\
\hline P01008 & ANT3 & FDTISEK \\
\hline P01008 & ANT3 & LQPLDFK \\
\hline P01008 & ANT3 & VAEGTQVLELPFK \\
\hline P01008 & ANT3 & VANPCVK \\
\hline P01008 & ANT3 & VWELSK \\
\hline P08519 & APOA & LFLEPTQADIALLK \\
\hline P08519 & APOA & NPDAVAAPYCYTR \\
\hline P08519 & APOA & NPDPVAAPYCYTR \\
\hline P02647 & APOA1 & AHVDALR \\
\hline P02647 & APOA1 & DLATVYVDVLK \\
\hline P02647 & APOA1 & EQLGPVTQEFWDNLEK \\
\hline P02647 & APOA1 & LLDNWDSVTSTFSK \\
\hline P02647 & APOA1 & QGLLPVLESFK \\
\hline P02647 & APOA1 & VQPYLDDFQK \\
\hline P02647 & APOA1 & VSFLSALEEYTK \\
\hline P02652 & APOA2 & EPCVESLVSQYFQTVTDYGK \\
\hline P02652 & APOA2 & SPELQAEAK \\
\hline P06727 & APOA4 & GNTEGLQK \\
\hline P06727 & APOA4 & IDQNVEELK \\
\hline
\end{tabular}

$\begin{array}{cccc}719.4 & 723.4 & 2 & 316.2 \\ 388.2 & 392.2 & 2 & 587.3 \\ 575 & 578.3 & 3 & 530.3 \\ 417.2 & 422.2 & 2 & 390.2 \\ 764.9 & 768.9 & 2 & 234.1 \\ 420.2 & 424.2 & 2 & 692.3 \\ 430.7 & 434.8 & 2 & 619.3 \\ 715.9 & 719.9 & 2 & 391.2 \\ 394.2 & 398.2 & 2 & 503.3 \\ 381.2 & 385.2 & 2 & 476.3 \\ 524.6 & 527.3 & 3 & 743.5 \\ 749.3 & 754.3 & 2 & 859.4 \\ 762.4 & 767.4 & 2 & 859.4 \\ 391.2 & 396.2 & 2 & 573.3 \\ 618.3 & 622.4 & 2 & 736.4 \\ 967 & 971 & 2 & 258.1 \\ 806.9 & 810.9 & 2 & 971.5 \\ 410.9 & 413.6 & 3 & 623.3 \\ 626.8 & 630.8 & 2 & 228.1 \\ 693.9 & 697.9 & 2 & 853.4 \\ 1175.5 & 1179.6 & 2 & 583.3 \\ 486.8 & 490.8 & 2 & 443.2 \\ 423.7 & 427.7 & 2 & 675.4 \\ 544.3 & 548.3 & 2 & 974.5\end{array}$

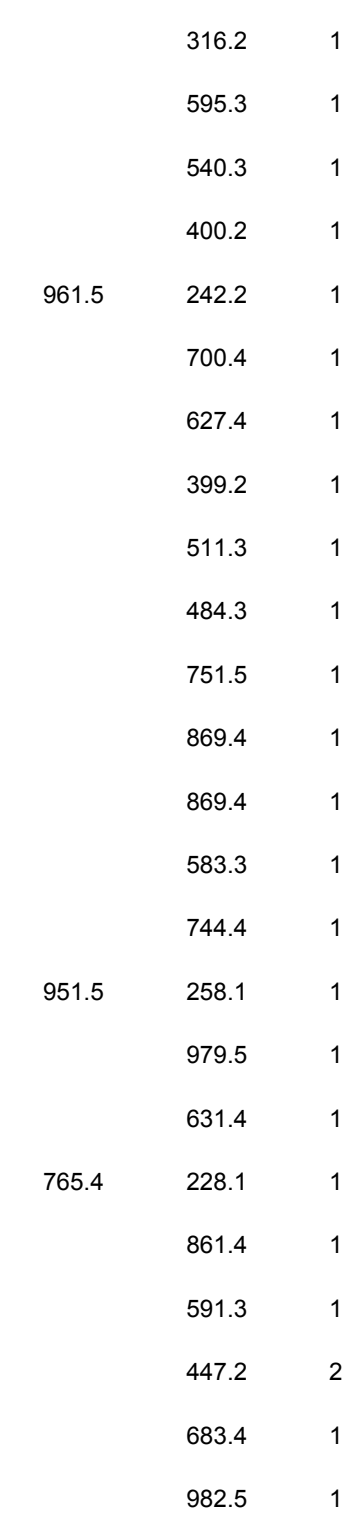

\begin{tabular}{|c|c|c|c|}
\hline b3 & & $Y$ & 23.3 \\
\hline y5 & & $Y$ & 13 \\
\hline y5 & & $Y$ & 15.9 \\
\hline y3 & & $Y$ & 13.9 \\
\hline y2 & y8 & $Y$ & 24.7 \\
\hline y6 & & $Y$ & 14 \\
\hline y5 & & $Y$ & 14.4 \\
\hline y3 & & $Y$ & 23.2 \\
\hline y4 & & $Y$ & 13.2 \\
\hline y4 & & $Y$ & 12.8 \\
\hline y7 & & $Y$ & 14.1 \\
\hline y6 & & $Y$ & 24.2 \\
\hline y6 & & $Y$ & 24.6 \\
\hline y5 & & $Y$ & 13.1 \\
\hline y6 & & $Y$ & 20.2 \\
\hline b2 & y7 & $Y$ & 31 \\
\hline y9 & & $Y$ & 26 \\
\hline y5 & & $Y$ & 10 \\
\hline b2 & y6 & $Y$ & 20.4 \\
\hline y7 & & $Y$ & 22.5 \\
\hline y5 & & $Y$ & 37.4 \\
\hline y8 & & $Y$ & 16.1 \\
\hline y6 & & $Y$ & 14.1 \\
\hline y8 & & $Y$ & 17.9 \\
\hline
\end{tabular}




\begin{tabular}{|l|l|l|} 
P06727 & APOA4 & IDQTVEELR \\
\hline P06727 & APOA4 & ISASAEELR \\
\hline P06727 & APOA4 & LGEVNTYAGDLQK \\
\hline P06727 & APOA4 & VEPYGENFNK \\
\hline P04114 & APOB & ALVDTLK \\
\hline P04114 & APOB & FSVPAGIVIPSFQALTAR \\
\hline P04114 & APOB & ITLPDFR \\
\hline P04114 & APOB & LATALSLSNK \\
\hline P04114 & APOB & QGFFPDSVNK \\
\hline P04114 & APOB & TSSFALNLPTLPEVK \\
\hline P02655 & APOC2 & ESLSSYWESAK \\
\hline P02655 & APOC2 & TAAQNLYEK \\
\hline P02655 & APOC2 & TYLPAVDEK \\
\hline P02656 & APOC3 & DALSSVQESQVAQQAR \\
\hline P02656 & APOC3 & DYWSTVK \\
\hline P02656 & APOC3 & GWVTDGFSSLK \\
\hline P05090 & APOD & CPNPPVQENFDVNK \\
\hline P05090 & APOD & IPTTFENGR \\
\hline P05090 & APOD & NILTSNNIDVK \\
\hline P05090 & APOD & NPNLPPETVDSLK \\
\hline P05090 & APOD & VLNQELR \\
\hline P02649 & APOE & AATVGSLAGQPLQER \\
\hline P02649 & APOE & AQAWGER \\
\hline P02649 & APOE & EQVAEVR \\
\hline
\end{tabular}

\begin{tabular}{|c|c|c|c|}
\hline 551.8 & 556.8 & 2 & 746.4 \\
\hline 488.3 & 493.3 & 2 & 775.4 \\
\hline 704.4 & 708.4 & 2 & 794.4 \\
\hline 598.8 & 602.8 & 2 & 484.7 \\
\hline 380.2 & 384.2 & 2 & 575.3 \\
\hline 937.5 & 942.5 & 2 & 1103.6 \\
\hline 431.2 & 436.2 & 2 & 534.3 \\
\hline 509.3 & 513.3 & 2 & 185.1 \\
\hline 569.8 & 573.8 & 2 & 659.3 \\
\hline 808.9 & 813 & 2 & 472.3 \\
\hline 643.8 & 647.8 & 2 & 957.4 \\
\hline 519.3 & 523.3 & 2 & 865.4 \\
\hline 518.3 & 522.3 & 2 & 658.3 \\
\hline 858.9 & 863.9 & 2 & 573.3 \\
\hline 449.7 & 453.7 & 2 & 620.3 \\
\hline 598.8 & 602.8 & 2 & 244.1 \\
\hline 829.4 & 833.4 & 2 & 643.8 \\
\hline 517.8 & 522.8 & 2 & 461.2 \\
\hline 615.8 & 619.8 & 2 & 228.1 \\
\hline 712.4 & 716.4 & 2 & 985.5 \\
\hline 436.3 & 441.3 & 2 & 659.3 \\
\hline 749.4 & 754.4 & 2 & 827.4 \\
\hline 409.2 & 414.2 & 2 & 618.3 \\
\hline 415.7 & 420.7 & 2 & 474.3 \\
\hline
\end{tabular}

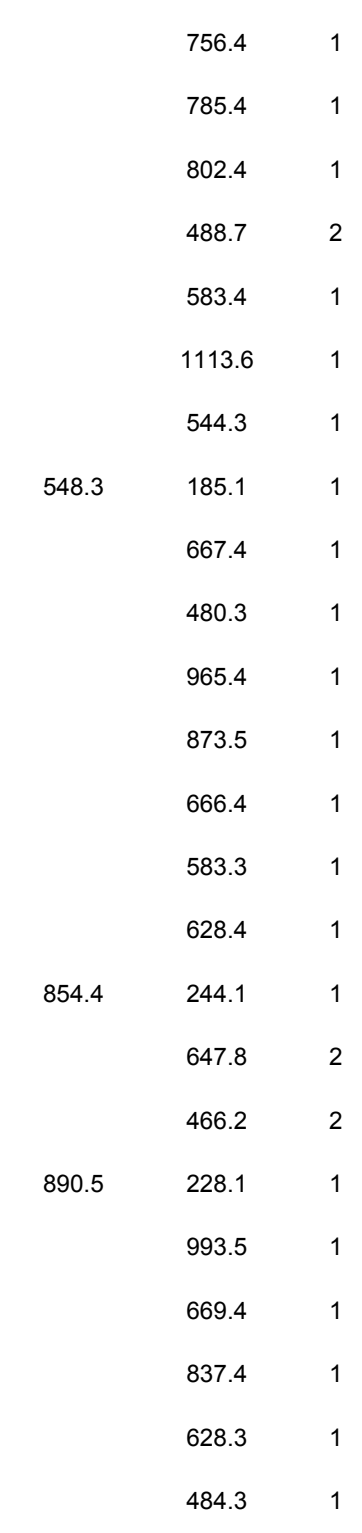

\begin{tabular}{|c|c|c|c|}
\hline y6 & & $Y$ & 18.1 \\
\hline y7 & & $\mathrm{Y}$ & 16.1 \\
\hline y7 & & $\mathrm{Y}$ & 22.8 \\
\hline y8 & & $\mathrm{Y}$ & 19.6 \\
\hline y5 & & $Y$ & 12.8 \\
\hline y10 & & $\mathrm{Y}$ & 30.1 \\
\hline y4 & & $Y$ & 14.4 \\
\hline b2 & y5 & $Y$ & 16.8 \\
\hline y6 & & $\mathrm{Y}$ & 18.7 \\
\hline y4 & & $\mathrm{Y}$ & 26.1 \\
\hline y8 & & $Y$ & 21 \\
\hline y7 & & $\mathrm{Y}$ & 17.1 \\
\hline y6 & & Y & 17.1 \\
\hline y5 & & $Y$ & 27.6 \\
\hline y5 & & Y & 14.9 \\
\hline b2 & y8 & $\mathrm{Y}$ & 19.6 \\
\hline y11 & & $Y$ & 26.7 \\
\hline y8 & & $Y$ & 17.1 \\
\hline b2 & y8 & $\mathrm{Y}$ & 20.1 \\
\hline y9 & & $\mathrm{Y}$ & 23.1 \\
\hline y5 & & $Y$ & 14.5 \\
\hline y7 & & $\mathrm{Y}$ & 24.2 \\
\hline y5 & & $\mathrm{Y}$ & 13.7 \\
\hline y4 & & $\mathrm{Y}$ & 13.9 \\
\hline
\end{tabular}




\begin{tabular}{|l|l|l|} 
P02649 & APOE & LAVYQAGAR \\
\hline P02649 & APOE & LQAEAFQAR \\
\hline P02649 & APOE & QQTEWQSGQR \\
\hline P02649 & APOE & QWAGLVEK \\
\hline Q13790 & APOF & SLPTEDCENEK \\
\hline P02749 & APOH & ATVVYQGER \\
\hline P02749 & APOH & CSYTEDAQCIDGTIEVPK \\
\hline P02749 & APOH & FICPLTGLWPINTLK \\
\hline P02749 & APOH & VCPFAGILENGAVR \\
\hline P02749 & APOH & VSFFCK \\
\hline O14791 & APOL1 & ALDNLAR \\
\hline O14791 & APOL1 & LNILNNNYK \\
\hline O14791 & APOL1 & VTEPISAESGEQVER \\
\hline O95445 & APOM & AFLLTPR \\
\hline O95445 & APOM & DGLCVPR \\
\hline O95445 & APOM & FLLYNR \\
\hline O95445 & APOM & SLTSCLDSK \\
\hline O95445 & APOM & WIYHLTEGSTDLR \\
\hline P00966 & ASSY & IDIVENR \\
\hline Q76LX8 & ATS13 & LFINVAPHAR \\
\hline P61769 & B2MG & VNHVTLSQPK \\
\hline P02730 & B3AT & LSVPDGFK \\
\hline Q8TDL5 & BPIB1 & ALGFEAAESSLTK \\
\hline P43251 & BTD & LSSGLVTAALYGR \\
\hline
\end{tabular}

\begin{tabular}{|c|c|c|c|}
\hline 474.8 & 479.8 & 2 & 665.3 \\
\hline 517.3 & 522.3 & 2 & 792.4 \\
\hline 624.3 & 629.3 & 2 & 761.4 \\
\hline 465.8 & 469.8 & 2 & 616.4 \\
\hline 661.3 & 665.3 & 2 & 1121.4 \\
\hline 511.8 & 516.8 & 2 & 652.3 \\
\hline 696 & 698.6 & 3 & 244.2 \\
\hline 887 & 891 & 2 & 421.2 \\
\hline 751.9 & 756.9 & 2 & 260.1 \\
\hline 394.2 & 398.2 & 2 & 688.3 \\
\hline 386.7 & 391.7 & 2 & 588.3 \\
\hline 553.3 & 557.3 & 2 & 228.1 \\
\hline 815.9 & 820.9 & 2 & 804.4 \\
\hline 409.3 & 414.3 & 2 & 599.4 \\
\hline 408.7 & 413.7 & 2 & 531.3 \\
\hline 413.2 & 418.2 & 2 & 565.3 \\
\hline 505.7 & 509.8 & 2 & 810.4 \\
\hline 530.9 & 534.3 & 3 & 288.2 \\
\hline 429.7 & 434.7 & 2 & 630.4 \\
\hline 379.9 & 383.2 & 3 & 480.3 \\
\hline 374.9 & 377.6 & 3 & 512.3 \\
\hline 431.7 & 435.7 & 2 & 563.3 \\
\hline 662.3 & 666.4 & 2 & 806.4 \\
\hline 654.4 & 659.4 & 2 & 751.4 \\
\hline
\end{tabular}

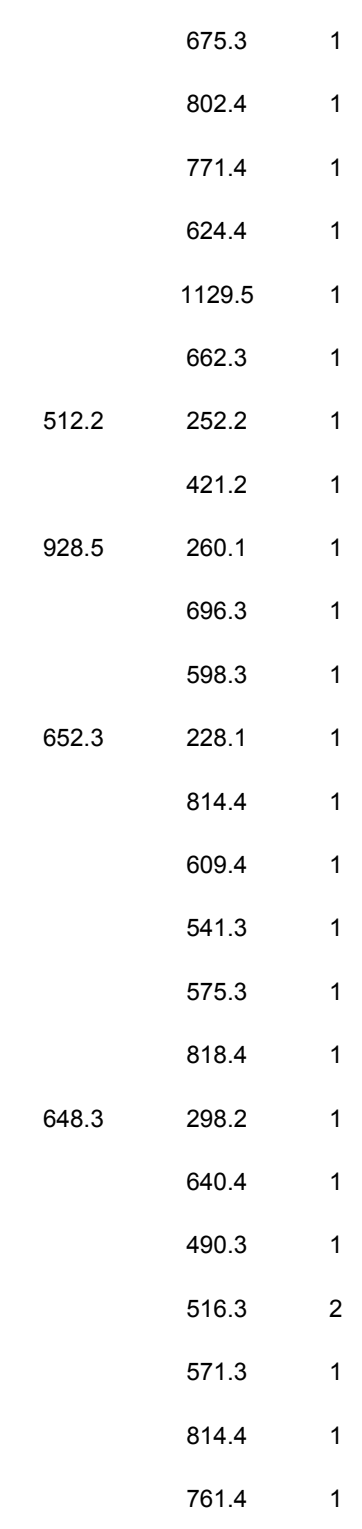

\begin{tabular}{|c|c|c|c|}
\hline y6 & & $Y$ & 15.7 \\
\hline y7 & & $Y$ & 17 \\
\hline y6 & & $Y$ & 20.4 \\
\hline y6 & & $Y$ & 15.4 \\
\hline y9 & & $Y$ & 21.5 \\
\hline y5 & & $Y$ & 16.9 \\
\hline y2 & b4 & $Y$ & 20.3 \\
\hline b3 & & $Y$ & 28.5 \\
\hline b2 & y9 & $Y$ & 24.3 \\
\hline y5 & & $Y$ & 13.2 \\
\hline y5 & & $Y$ & 13 \\
\hline b2 & y5 & $Y$ & 18.2 \\
\hline y7 & & $Y$ & 26.3 \\
\hline y5 & & $Y$ & 13.7 \\
\hline y4 & & $Y$ & 13.7 \\
\hline y4 & & $Y$ & 13.8 \\
\hline y7 & & $Y$ & 16.7 \\
\hline y2 & y6 & $Y$ & 14.3 \\
\hline y5 & & $Y$ & 14.3 \\
\hline y4 & & $Y$ & 8.9 \\
\hline y9 & & $Y$ & 8.7 \\
\hline y5 & & $Y$ & 14.4 \\
\hline y8 & & $Y$ & 21.5 \\
\hline y7 & & $Y$ & 21.3 \\
\hline
\end{tabular}




\begin{tabular}{|l|l|l|} 
P43251 & BTD & SHLIIAQVAK \\
\hline P43251 & BTD & VDLITFDTPFAGR \\
\hline Q06187 & BTK & LVQLYGVCTK \\
\hline P02745 & C1QA & SLGFCDTTNK \\
\hline P02746 & C1QB & IAFSATR \\
\hline P02746 & C1QB & LEQGENVFLQATDK \\
\hline P02746 & C1QB & TINVPLR \\
\hline P02747 & C1QC & FNAVLTNPQGDYDTSTGK \\
\hline P02747 & C1QC & FQSVFTVTR \\
\hline P02747 & C1QC & QTHQPPAPNSLIR \\
\hline P02747 & C1QC & TNQVNSGGVLLR \\
\hline P02747 & C1QC & VVTFCGHTSK \\
\hline P00736 & C1R & FCGQLGSPLGNPPGK \\
\hline P00736 & C1R & GYGFYTK \\
\hline P00736 & C1R & NIGEFCGK \\
\hline P00736 & C1R & QDACQGDSGGVFAVR \\
\hline P00736 & C1R & VLNYVDWIK \\
\hline P00736 & C1R & YTTEIIK \\
\hline Q9NZP8 & C1RL & GSEAINAPGDNPAK \\
\hline Q9NZP8 & C1RL & WILTAAHTIYPK \\
\hline P09871 & C1S & CEYQIR \\
\hline P09871 & C1S & IIGGSDADIK \\
\hline P09871 & C1S & LQVIFK \\
\hline P09871 & C1S & SNALDIIFQTDLTGQK \\
\hline
\end{tabular}

$\begin{array}{llll}360.6 & 363.2 & 3 & 451.3 \\ 484.6 & 487.9 & 3 & 274.2 \\ 590.8 & 594.8 & 2 & 727.3 \\ 571.8 & 575.8 & 2 & 942.4 \\ 383.2 & 388.2 & 2 & 652.3 \\ 531.3 & 533.9 & 3 & 822.4 \\ 406.8 & 411.8 & 2 & 598.4 \\ 964.5 & 968.5 & 2 & 333.2 \\ 542.8 & 547.8 & 2 & 809.5 \\ 486.9 & 490.3 & 3 & 350.2 \\ 629.3 & 634.4 & 2 & 815.5 \\ 379.2 & 381.9 & 3 & 199.1 \\ 764.9 & 768.9 & 2 & 398.2 \\ 418.2 & 422.2 & 2 & 615.3 \\ 462.7 & 466.7 & 2 & 697.3 \\ 783.9 & 788.9 & 2 & 964.5 \\ 575.3 & 579.3 & 2 & 937.5 \\ 434.2 & 438.3 & 2 & 603.4 \\ 670.8 & 674.8 & 2 & 698.3 \\ 707.4 & 711.4 & 2 & 1114.6 \\ 434.7 & 439.7 & 2 & 579.3 \\ 494.8 & 498.8 & 2 & 762.4 \\ 374.2 & 378.2 & 2 & 506.3 \\ 882.5 & 886.5 & 2 & 273.1\end{array}$

$\begin{array}{ll}451.3 & 1 \\ 279.2 & 2 \\ 735.4 & 1 \\ 950.4 & 1 \\ 662.3 & 1 \\ 830.4 & 1 \\ 608.4 & 1 \\ 333.2 & 1 \\ 819.5 & 1 \\ 355.2 & 2 \\ 825.5 & 1 \\ 199.1 & 1 \\ 406.3 & 1 \\ 623.3 & 1 \\ 705.3 & 1 \\ 974.5 & 1 \\ 945.5 & 1 \\ 611.4 & 1 \\ 706.4 & 1 \\ 1122.6 & 1 \\ 589.3 & 1 \\ 770.4 & 1 \\ 514.3 & 1 \\ 273.1 & 1\end{array}$




\begin{tabular}{|l|l|l|} 
P09871 & C1S & SSNNPHSPIVEEFQVPYNK \\
\hline P09871 & C1S & TNFDNDIALVR \\
\hline P04003 & C4BPA & FSAICQGDGTWSPR \\
\hline P04003 & C4BPA & LSCSYSHWSAPAPQCK \\
\hline P04003 & C4BPA & YTCLPGYVR \\
\hline P54289 & CA2D1 & VLLDAGFTNELVQNYWSK \\
\hline P00915 & CAH1 & ESISVSSEQLAQFR \\
\hline P00915 & CAH1 & GGPFSDSYR \\
\hline P00915 & CAH1 & YSSLAEAASK \\
\hline P00918 & CAH2 & YGDFGK \\
\hline P27797 & CALR & FVLSSGK \\
\hline P27797 & CALR & QIDNPDYK \\
\hline P08185 & CBG & HLVALSPK \\
\hline P08185 & CBG & ITQDAQLK \\
\hline P08185 & CBG & QINSYVK \\
\hline P22681 & CBL & GTEPIVVDPFDPR \\
\hline Q96IY4 & CBPB2 & DTGTYGFLLPER \\
\hline Q96IY4 & CBPB2 & YPLYVLK \\
\hline P15169 & CBPN & IVQLIQDTR \\
\hline P15169 & CBPN & VQNECPGITR \\
\hline P15169 & CBPN & VYSIGR \\
\hline P15169 & CBPN & YDDLVR \\
\hline P15169 & CBPN & YGGPNHHLPLPDNWK \\
\hline P30279 & CCND2 & ACQEQIEAVLLNSLQQYR \\
\hline
\end{tabular}

\begin{tabular}{|c|c|c|c|}
\hline 729.4 & 732 & 3 & 521.3 \\
\hline 639.3 & 644.3 & 2 & 915.5 \\
\hline 791.4 & 796.4 & 2 & 875.4 \\
\hline 626.9 & 629.6 & 3 & 700.3 \\
\hline 564.8 & 569.8 & 2 & 591.3 \\
\hline 699.7 & 702.4 & 3 & 825.4 \\
\hline 790.9 & 795.9 & 2 & 1065.5 \\
\hline 493.2 & 498.2 & 2 & 774.3 \\
\hline 513.8 & 517.8 & 2 & 776.4 \\
\hline 343.7 & 347.7 & 2 & 523.3 \\
\hline 369.2 & 373.2 & 2 & 491.3 \\
\hline 496.7 & 500.7 & 2 & 522.3 \\
\hline 432.8 & 436.8 & 2 & 251.2 \\
\hline 458.8 & 462.8 & 2 & 702.4 \\
\hline 426.2 & 430.2 & 2 & 610.3 \\
\hline 721.4 & 726.4 & 2 & 577.8 \\
\hline 684.8 & 689.8 & 2 & 401.2 \\
\hline 448.3 & 452.3 & 2 & 366.7 \\
\hline 543.3 & 548.3 & 2 & 873.5 \\
\hline 587.3 & 592.3 & 2 & 946.4 \\
\hline 347.7 & 352.7 & 2 & 432.3 \\
\hline 390.7 & 395.7 & 2 & 502.3 \\
\hline 582.3 & 585 & 3 & 659.3 \\
\hline 721.7 & 725 & 3 & 908.5 \\
\hline
\end{tabular}

$\begin{array}{cl}529.3 & 1 \\ 925.5 & 1 \\ 885.4 & 1 \\ 708.4 & 1 \\ 601.3 & 1 \\ 833.4 & 1 \\ 1075.5 & 1 \\ 784.3 & 1 \\ 784.4 & 1 \\ 531.3 & 1 \\ 499.3 & 1 \\ 530.3 & 1 \\ 251.2 & 1 \\ 710.4 & 1 \\ 618.3 & 1 \\ 582.8 & 2 \\ 411.2 & 1 \\ 370.7 & 2 \\ 883.5 & 1 \\ 956.4 & 1 \\ 442.3 & 1 \\ 512.3 & 1 \\ 6667.3 & 1 \\ 918.5 & 1\end{array}$

\begin{tabular}{|c|c|c|c|}
\hline y4 & & $Y$ & 21.5 \\
\hline y8 & & $Y$ & 20.8 \\
\hline y8 & & $Y$ & 25.5 \\
\hline y6 & & $Y$ & 17.8 \\
\hline y5 & & $Y$ & 18.5 \\
\hline y6 & & $Y$ & 20.4 \\
\hline y9 & & $Y$ & 25.5 \\
\hline y6 & & $Y$ & 16.3 \\
\hline y8 & & $Y$ & 16.9 \\
\hline y5 & & $Y$ & 11.7 \\
\hline y5 & & $Y$ & 12.4 \\
\hline y4 & & $Y$ & 16.4 \\
\hline b2 & y6 & $Y$ & 14.4 \\
\hline y6 & & $Y$ & 15.2 \\
\hline y5 & & $Y$ & 14.2 \\
\hline y10 & & $Y$ & 23.4 \\
\hline y3 & & $Y$ & 22.2 \\
\hline y6 & & $Y$ & 14.9 \\
\hline y7 & & $Y$ & 17.8 \\
\hline y8 & & $Y$ & 19.2 \\
\hline y4 & & $Y$ & 11.8 \\
\hline y4 & & $Y$ & 13.1 \\
\hline y5 & & $Y$ & 16.2 \\
\hline y7 & & $Y$ & 21.2 \\
\hline
\end{tabular}




\begin{tabular}{|l|l|l|} 
P08571 & CD14 & ATVNPSAPR \\
\hline P08571 & CD14 & VLAYSR \\
\hline P08571 & CD14 & VLDLSCNR \\
\hline O43866 & CD5L & CYGPGVGR \\
\hline O43866 & CD5L & LVGGDNLCSGR \\
\hline P06731 & CEAM5 & TLTLFNVTR \\
\hline P00450 & CERU & DIASGLIGPLIICK \\
\hline P00450 & CERU & EVGPTNADPVCLAK \\
\hline P00450 & CERU & EYTDASFTNR \\
\hline P00450 & CERU & GAYPLSIEPIGVR \\
\hline P00450 & CERU & IGGSYK \\
\hline P00450 & CERU & QSEDSTFYLGER \\
\hline P00751 & CFAB & CLVNLIEK \\
\hline P00751 & CFAB & DISEVVTPR \\
\hline P00751 & CFAB & STGSWSTLK \\
\hline P00751 & CFAB & VSEADSSNADWVTK \\
\hline P00751 & CFAB & YGLVTYATYPK \\
\hline P08603 & CFAH & CVEISCK \\
\hline P08603 & CFAH & DGWSAQPTCIK \\
\hline P08603 & CFAH & EYHFGQAVR \\
\hline P08603 & CFAH & TGDEITYQCR \\
\hline P08603 & CFAH & TGESVEFVCK \\
\hline P08603 & CFAH & VGEVLK \\
\hline P08603 & CFAH & WQSIPLCVEK \\
\hline
\end{tabular}

$\begin{array}{llll}456.7 & 461.8 & 2 & 527.3 \\ 354.7 & 359.7 & 2 & 496.3 \\ 488.7 & 493.8 & 2 & 764.3 \\ 433.2 & 438.2 & 2 & 542.3 \\ 574.3 & 579.3 & 2 & 935.4 \\ 532.8 & 537.8 & 2 & 850.5 \\ 735.4 & 739.4 & 2 & 800.5 \\ 735.9 & 739.9 & 2 & 802.4 \\ 602.3 & 607.3 & 2 & 624.3 \\ 457.9 & 461.3 & 3 & 541.3 \\ 312.7 & 316.7 & 2 & 511.3 \\ 716.3 & 721.3 & 2 & 637.3 \\ 494.8 & 498.8 & 2 & 715.4 \\ 508.3 & 513.3 & 2 & 787.4 \\ 483.7 & 487.8 & 2 & 778.4 \\ 754.8 & 758.9 & 2 & 248.2 \\ 638.3 & 642.3 & 2 & 843.4 \\ 448.2 & 452.2 & 2 & 636.3 \\ 631.8 & 635.8 & 2 & 904.5 \\ 369.5 & 372.9 & 3 & 274.2 \\ 621.8 & 626.8 & 2 & 727.3 \\ 578.3 & 582.3 & 2 & 682.3 \\ 322.7 & 326.7 & 2 & 545.3 \\ 630.3 & 634.3 & 2 & 745.4\end{array}$

\begin{tabular}{|c|c|c|}
\hline & 537.3 & 1 \\
\hline & 506.3 & 1 \\
\hline & 774.3 & 1 \\
\hline & 552.3 & 1 \\
\hline & 945.4 & 1 \\
\hline & 860.5 & 1 \\
\hline & 808.5 & 1 \\
\hline & 810.4 & 1 \\
\hline & 634.3 & 1 \\
\hline & 551.4 & 1 \\
\hline & 519.3 & 1 \\
\hline & 647.3 & 1 \\
\hline & 723.4 & 1 \\
\hline & 797.4 & 1 \\
\hline & 786.4 & 1 \\
\hline 920.4 & 256.2 & 1 \\
\hline & 851.4 & 1 \\
\hline & 644.3 & 1 \\
\hline & 912.5 & 1 \\
\hline 530.3 & 284.2 & 1 \\
\hline & 737.3 & 1 \\
\hline & 690.3 & 1 \\
\hline & 553.3 & 1 \\
\hline & 753.4 & 1 \\
\hline
\end{tabular}

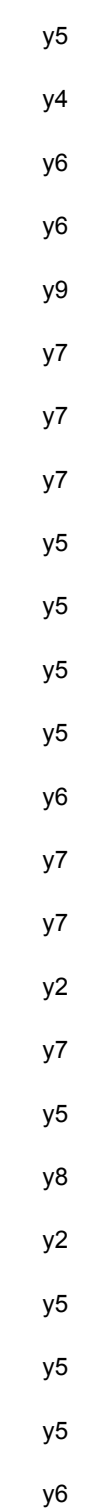

\begin{tabular}{c|}
15.2 \\
12 \\
16.2 \\
14.4 \\
18.8 \\
17.5 \\
23.8 \\
23.8 \\
19.7 \\
11.7 \\
10.7 \\
23.2 \\
16.3 \\
16.8 \\
16 \\
24.4 \\
20.8 \\
14.9 \\
20.6 \\
8.5 \\
\hline 20.3 \\
18.9 \\
\hline 11 \\
\hline 20.5 \\
\hline 16
\end{tabular}




\begin{tabular}{|l|l|l|} 
P05156 & CFAI & ACDGINDCGDQSDELCCK \\
\hline P05156 & CFAI & AQLGDLPWQVAIK \\
\hline P05156 & CFAI & EANVACLDLGFQGADTQR \\
\hline P05156 & CFAI & GLETSLAECTFTK \\
\hline P05156 & CFAI & HGNTDSEGIVEVK \\
\hline P06276 & CHLE & AEEILSR \\
\hline P06276 & CHLE & IFFPGVSEFGK \\
\hline P06276 & CHLE & TQILVGVNK \\
\hline P10909 & CLUS & ASSIIDELFQDR \\
\hline P10909 & CLUS & EIQNAVNGVK \\
\hline P10909 & CLUS & ELDESLQVAER \\
\hline P10909 & CLUS & TLLSNLEEAK \\
\hline Q96KN2 & CNDP1 & AIHLDLEEYR \\
\hline Q96KN2 & CNDP1 & EWVAIESDSVQPVPR \\
\hline P02452 & CO1A1 & VLCDDVICDETK \\
\hline P06681 & CO2 & AVISPGFDVFAK \\
\hline P06681 & CO2 & CSSNLVLTGSSER \\
\hline P06681 & CO2 & EILNINQK \\
\hline P06681 & CO2 & GALISDQWVLTAAHCFR \\
\hline P06681 & CO2 & HAFILQDTK \\
\hline P06681 & CO2 & LNINLK \\
\hline P06681 & CO2 & SSGQWQTPGATR \\
\hline P01024 & CO3 & ACEPGVDYVYK \\
\hline P01024 & CO3 & DSCVGSLVVK \\
\hline
\end{tabular}

\begin{tabular}{|c|c|c|c|}
\hline 706.3 & 708.9 & 3 & 467.2 \\
\hline 719.9 & 723.9 & 2 & 200.1 \\
\hline 698.3 & 701.7 & 3 & 775.4 \\
\hline 728.9 & 732.9 & 2 & 856.4 \\
\hline 462.2 & 464.9 & 3 & 644.4 \\
\hline 409.2 & 414.2 & 2 & 617.4 \\
\hline 614.3 & 618.3 & 2 & 820.4 \\
\hline 486.3 & 490.3 & 2 & 230.1 \\
\hline 697.4 & 702.4 & 2 & 922.4 \\
\hline 536.3 & 540.3 & 2 & 701.4 \\
\hline 644.8 & 649.8 & 2 & 802.4 \\
\hline 559.3 & 563.3 & 2 & 215.1 \\
\hline 420.2 & 423.6 & 3 & 467.2 \\
\hline 856.4 & 861.4 & 2 & 468.3 \\
\hline 733.8 & 737.8 & 2 & 627.8 \\
\hline 625.8 & 629.8 & 2 & 880.5 \\
\hline 705.3 & 710.3 & 2 & 749.4 \\
\hline 486.3 & 490.3 & 2 & 616.3 \\
\hline 649 & 652.3 & 3 & 242.1 \\
\hline 536.8 & 540.8 & 2 & 209.1 \\
\hline 357.7 & 361.7 & 2 & 601.4 \\
\hline 638.3 & 643.3 & 2 & 501.3 \\
\hline 650.8 & 654.8 & 2 & 940.5 \\
\hline 532.3 & 536.3 & 2 & 602.4 \\
\hline
\end{tabular}

$\begin{array}{lll} & 475.2 & 1 \\ 841.5 & 200.1 & 1 \\ & 785.4 & 1 \\ & 864.4 & 1 \\ & 652.4 & 1 \\ & 627.4 & 1 \\ 629.4 & 828.4 & 1 \\ & 230.1 & 1 \\ & 932.4 & 1 \\ & 709.4 & 1 \\ & 812.5 & 1 \\ & 215.1 & 1 \\ & 477.2 & 1 \\ & 478.3 & 1 \\ & 631.8 & 2 \\ & & \\ & 888.5 & 1 \\ & 759.4 & 1 \\ & 624.4 & 1 \\ & 242.1 & 1 \\ & 209.1 & 1 \\ 609.4 & 1 \\ & 511.3 & 1 \\ & 948.5 & 1 \\ & 610.4 & 1\end{array}$

1




\begin{tabular}{|l|l|l|} 
P01024 & CO3 & IWDVVEK \\
\hline P01024 & CO3 & NEQVEIR \\
\hline P01024 & CO3 & VLLDGVQNPR \\
\hline P0C0L4 & CO4A & ANSFLGEK \\
\hline P0C0L4 & CO4A & DSSTWLTAFVLK \\
\hline P08572 & CO4A2 & IAVQPGTVGPQGR \\
\hline P01031 & CO5 & AFDICPLVK \\
\hline P01031 & CO5 & EYVLPHFSVSIEPEYNFIGYK \\
\hline P01031 & CO5 & IDTALIK \\
\hline P01031 & CO5 & IDTQDIEASHYR \\
\hline P01031 & CO5 & ITHYNYLILSK \\
\hline P01031 & CO5 & NFEITIK \\
\hline P01031 & CO5 & QLPGGQNPVSYVYLEVVSK \\
\hline P01031 & CO5 & TLLPVSKPEIR \\
\hline P01031 & CO5 & VFQFLEK \\
\hline P13671 & CO6 & ALNHLPLEYNSALYSR \\
\hline P13671 & CO6 & DLHLSDVFLK \\
\hline P13671 & CO6 & ENPAVIDFELAPIVDLVR \\
\hline P13671 & CO6 & GEVLDNSFTGGICK \\
\hline P13671 & CO6 & GFVVAGPSR \\
\hline P13671 & CO6 & SEYGAALAWEK \\
\hline P13671 & CO6 & TLNICEVGTIR \\
\hline P10643 & CO7 & AASGTQNNVLR \\
\hline P10643 & CO7 & DSCTLPASAEK \\
\hline
\end{tabular}

\begin{tabular}{|c|c|c|c|}
\hline 444.7 & 448.8 & 2 & 589.3 \\
\hline 444.2 & 449.2 & 2 & 644.4 \\
\hline 555.8 & 560.8 & 2 & 898.5 \\
\hline 433.2 & 437.2 & 2 & 446.3 \\
\hline 684.4 & 688.4 & 2 & 791.5 \\
\hline 427.2 & 430.6 & 3 & 457.3 \\
\hline 531.8 & 535.8 & 2 & 844.5 \\
\hline 844.4 & 847.1 & 3 & 1130.6 \\
\hline 387.2 & 391.2 & 2 & 660.4 \\
\hline 483.2 & 486.6 & 3 & 667.8 \\
\hline 455.6 & 458.3 & 3 & 460.3 \\
\hline 432.7 & 436.8 & 2 & 603.4 \\
\hline 1039.1 & 1043.1 & 2 & 837.5 \\
\hline 418.3 & 421.6 & 3 & 463.3 \\
\hline 455.8 & 459.8 & 2 & 664.4 \\
\hline 621 & 624.3 & 3 & 538.3 \\
\hline 593.8 & 597.8 & 2 & 479.8 \\
\hline 1005.5 & 1010.6 & 2 & 811.5 \\
\hline 748.9 & 752.9 & 2 & 286.1 \\
\hline 445.2 & 450.3 & 2 & 487.3 \\
\hline 612.8 & 616.8 & 2 & 845.5 \\
\hline 638.3 & 643.3 & 2 & 834.4 \\
\hline 565.8 & 570.8 & 2 & 143.1 \\
\hline 589.8 & 593.8 & 2 & 602.3 \\
\hline
\end{tabular}

\begin{tabular}{ll}
597.3 & 1 \\
654.4 & 1 \\
908.5 & 1 \\
454.3 & 1 \\
799.5 & 1 \\
467.3 & 1 \\
852.5 & 1 \\
11138.6 & 1 \\
668.4 & 1 \\
672.8 & 2 \\
4668.3 & 1 \\
611.4 & 1 \\
\hline 845.5 & 1 \\
4668.3 & 2 \\
672.4 & 1 \\
\hline 548.3 & 1 \\
483.8 & 2 \\
821.5 & 1 \\
\hline 286.1 & 1 \\
4997.3 & 1 \\
853.5 & 1 \\
844.4 & 1 \\
143.1 & 1 \\
610.3 & 1 \\
\hline
\end{tabular}

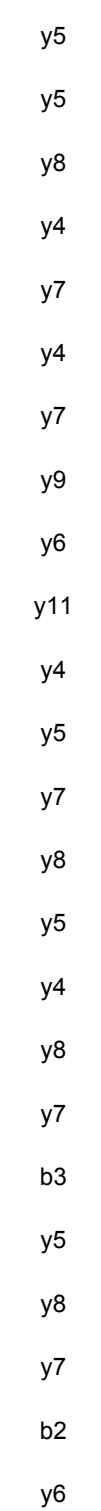

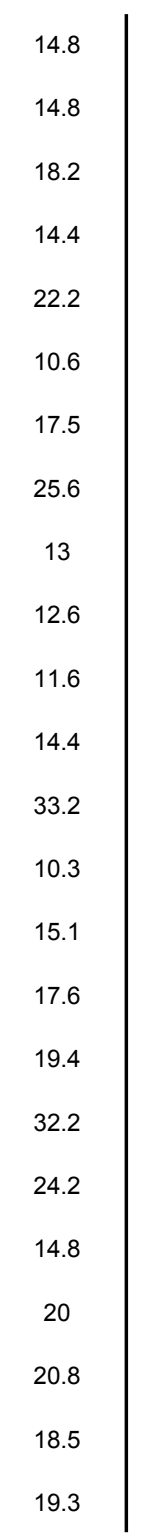




\begin{tabular}{|l|l|l|} 
P10643 & CO7 & ELSHLPSLYDYSAYR \\
\hline P10643 & CO7 & SCVGETTESTQCEDEELEHL \\
\hline P10643 & CO7 & VLFYVDSEK \\
\hline P10643 & CO7 & YSAWAESVTNLPQVIK \\
\hline P07357 & CO8A & AIDEDCSQYEPIPGSQK \\
\hline P07357 & CO8A & LGSLGAACEQTQTEGAK \\
\hline P07357 & CO8A & LYYGDDEK \\
\hline P07357 & CO8A & STITYR \\
\hline P07358 & CO8B & CEGFVCAQTGR \\
\hline P07358 & CO8B & IPGIFELGISSQSDR \\
\hline P07358 & CO8B & LPLEYSYGEYR \\
\hline P07358 & CO8B & QALEEFQK \\
\hline P07358 & CO8B & SGFSFGFK \\
\hline P07358 & CO8B & SVFLHAR \\
\hline P07360 & CO8G & AGQLSVK \\
\hline P07360 & CO8G & LDGICWQVR \\
\hline P07360 & CO8G & QLYGDTGVLGR \\
\hline P07360 & CO8G & SLPVSDSVLSGFEQR \\
\hline P07360 & CO8G & YGFCEAADQFHVLDEVR \\
\hline P02748 & CO9 & CLCACPFK \\
\hline P02748 & CO9 & FEGIACEISK \\
\hline P02748 & CO9 & SIEVFGQFNGK \\
\hline P02748 & CO9 & TSNFNAAISLK \\
\hline P02748 & CO9 & VVEESELAR \\
\hline
\end{tabular}

\begin{tabular}{|c|c|c|c|}
\hline 605.3 & 608.6 & 3 & 774.3 \\
\hline 837 & 840.4 & 3 & 248.1 \\
\hline 550.3 & 554.3 & 2 & 887.4 \\
\hline 903.5 & 907.5 & 2 & 584.4 \\
\hline 968.9 & 972.9 & 2 & 516.3 \\
\hline 860.9 & 864.9 & 2 & 275.2 \\
\hline 501.7 & 505.7 & 2 & 726.3 \\
\hline 370.7 & 375.7 & 2 & 552.3 \\
\hline 642.8 & 647.8 & 2 & 692.3 \\
\hline 809.9 & 814.9 & 2 & 849.4 \\
\hline 695.3 & 700.3 & 2 & 211.1 \\
\hline 496.8 & 500.8 & 2 & 680.3 \\
\hline 438.7 & 442.7 & 2 & 585.3 \\
\hline 415.2 & 420.2 & 2 & 643.4 \\
\hline 351.7 & 355.7 & 2 & 631.4 \\
\hline 573.8 & 578.8 & 2 & 748.4 \\
\hline 589.8 & 594.8 & 2 & 774.4 \\
\hline 810.9 & 815.9 & 2 & 836.4 \\
\hline 686 & 689.3 & 3 & 221.1 \\
\hline 528.2 & 532.2 & 2 & 782.3 \\
\hline 577.3 & 581.3 & 2 & 877.4 \\
\hline 613.3 & 617.3 & 2 & 797.4 \\
\hline 583.3 & 587.3 & 2 & 716.4 \\
\hline 516.3 & 521.3 & 2 & 833.4 \\
\hline
\end{tabular}

$\begin{array}{lll} & 784.3 & 1 \\ 425.3 & 248.1 & 1 \\ & 895.4 & 1 \\ & 592.4 & 1 \\ & 524.3 & 1 \\ & 283.2 & 1 \\ & 734.3 & 1 \\ & 562.3 & 1 \\ 774.3 & 702.3 & 1 \\ & 859.4 & 1 \\ & 211.1 & 1 \\ 688.3 & 1 \\ 593.3 & 1 \\ 653.4 & 1 \\ 639.4 & 1 \\ 758.4 & 1 \\ 784.4 & 1 \\ 846.4 & 1 \\ & 221.1 & 1 \\ 790.3 & 1 \\ 885.5 & 1 \\ 805.4 & 1 \\ 724.4 & 1 \\ 843.4 & 1\end{array}$




\begin{tabular}{|l|l|l|}
\hline Q03692 & COAA1 & GTHVWVGLYK \\
\hline Q9UMD9 & COHA1 & QAAYNADSGLK \\
\hline P49747 & COMP & DTDLDGFPDEK \\
\hline P49747 & COMP & EITFLK \\
\hline P20815 & CP3A5 & DTINFLSK \\
\hline P22792 & CPN2 & DHLGFQVTWPDESK \\
\hline P22792 & CPN2 & GQVVPALNEK \\
\hline P22792 & CPN2 & LTVSIEAR \\
\hline P22792 & CPN2 & QLVCPVTR \\
\hline P02741 & CRP & ESDTSYVSLK \\
\hline P02741 & CRP & GYSIFSYATK \\
\hline P02775 & CXCL7 & GTHCNQVEVIATLK \\
\hline P02775 & CXCL7 & ICLDPDAPR \\
\hline P02775 & CXCL7 & NIQSLEVIGK \\
\hline P02775 & CXCL7 & TTSGIHPK \\
\hline P01034 & CYTC & ALDFAVGEYNK \\
\hline O95822 & DCMC & LCAWYLYGEK \\
\hline P09172 & DOPO & VISTLEEPTPQCPTSQGR \\
\hline Q14126 & DSG2 & ILDVNDNIPVVENK \\
\hline P32926 & DSG3 & LAEISLGVDGEGK \\
\hline Q16610 & ECM1 & ELPSLQHPNEQK \\
\hline Q16610 & ECM1 & EVGPPLPQEAVPLQK \\
\hline Q16610 & ECM1 & FCEAEFSVK \\
\hline Q16610 & ECM1 & FSCFQEEAPQPHYQLR \\
\hline
\end{tabular}

\begin{tabular}{|c|c|c|c|}
\hline 387.2 & 389.9 & 3 & 480.3 \\
\hline 569.3 & 573.3 & 2 & 704.4 \\
\hline 626.3 & 630.3 & 2 & 488.2 \\
\hline 375.7 & 379.7 & 2 & 508.3 \\
\hline 469.3 & 473.3 & 2 & 721.4 \\
\hline 553.6 & 556.3 & 3 & 575.3 \\
\hline 527.8 & 531.8 & 2 & 671.4 \\
\hline 444.8 & 449.8 & 2 & 575.3 \\
\hline 486.8 & 491.8 & 2 & 731.4 \\
\hline 564.8 & 568.8 & 2 & 347.2 \\
\hline 568.8 & 572.8 & 2 & 716.4 \\
\hline 523.9 & 526.6 & 3 & 773.5 \\
\hline 528.8 & 533.8 & 2 & 783.4 \\
\hline 550.8 & 554.8 & 2 & 873.5 \\
\hline 420.7 & 424.7 & 2 & 638.4 \\
\hline 613.8 & 617.8 & 2 & 780.4 \\
\hline 651.8 & 655.8 & 2 & 772.4 \\
\hline 1000.5 & 1005.5 & 2 & 1228.6 \\
\hline 528 & 530.6 & 3 & 685.4 \\
\hline 644.3 & 648.4 & 2 & 861.4 \\
\hline 473.9 & 476.6 & 3 & 540.8 \\
\hline 801.4 & 805.5 & 2 & 485.3 \\
\hline 558.8 & 562.8 & 2 & 809.4 \\
\hline 679.6 & 683 & 3 & 519.8 \\
\hline
\end{tabular}

\begin{tabular}{ll}
488.3 & 1 \\
712.4 & 1 \\
496.2 & 1 \\
516.3 & 1 \\
729.4 & 1 \\
583.3 & 1 \\
679.4 & 1 \\
585.3 & 1 \\
741.4 & 1 \\
355.2 & 1 \\
724.4 & 1 \\
781.5 & 1 \\
793.4 & 1 \\
881.5 & 1 \\
646.4 & 1 \\
788.4 & 1 \\
780.4 & 1 \\
1238.6 & 1 \\
693.4 & 1 \\
869.4 & 1 \\
544.8 & 2 \\
493.3 & 1 \\
817.4 & 1 \\
524.8 & 2 \\
\hline 5
\end{tabular}




\begin{tabular}{|l|l|l|} 
Q16610 & ECM1 & LTFINDLCGPR \\
\hline Q16610 & ECM1 & NVALVSGDTENAK \\
\hline Q16610 & ECM1 & QHVVYGPWNLPQSSYSHLTR \\
\hline P00533 & EGFR & CNLLEGEPR \\
\hline Q01780 & EXOSX & SGPLPSAER \\
\hline P00488 & F13A & CGPASVQAIK \\
\hline P00488 & F13A & FQEGQEEER \\
\hline P00488 & F13A & GTYIPVPIVSELQSGK \\
\hline P00488 & F13A & LSIQSSPK \\
\hline P00488 & F13A & STVLTIPEIIIK \\
\hline P05160 & F13B & GDTYPAELYITGSILR \\
\hline P05160 & F13B & IQTHSTTYR \\
\hline P05160 & F13B & SGYLLHGSNEITCNR \\
\hline P05160 & F13B & VACEEPPFIENGAANLHSK \\
\hline P05160 & F13B & VLHGDLIDFVCK \\
\hline P05160 & F13B & VQYECATGYYTAGGK \\
\hline P00742 & FA10 & ACIPTGPYPCGK \\
\hline P00742 & FA10 & GYTLADNGK \\
\hline P00742 & FA10 & NCELFTR \\
\hline P00742 & FA10 & QEDACQGDSGGPHVTR \\
\hline P00742 & FA10 & TGIVSGFGR \\
\hline P00742 & FA10 & TNEFWNK \\
\hline P03951 & FA11 & ALSGFSLQSCR \\
\hline P03951 & FA11 & GGISGYTLR \\
\hline
\end{tabular}

\begin{tabular}{|c|c|c|c|}
\hline 653.3 & 658.3 & 2 & 831.4 \\
\hline 659.3 & 663.3 & 2 & 821.4 \\
\hline 790.4 & 793.7 & 3 & 588.3 \\
\hline 544.3 & 549.3 & 2 & 272.2 \\
\hline 457.2 & 462.2 & 2 & 559.3 \\
\hline 515.8 & 519.8 & 2 & 813.5 \\
\hline 576.3 & 581.3 & 2 & 876.4 \\
\hline 844.5 & 848.5 & 2 & 322.1 \\
\hline 430.2 & 434.3 & 2 & 746.4 \\
\hline 663.9 & 667.9 & 2 & 712.5 \\
\hline 885 & 890 & 2 & 922.5 \\
\hline 369.5 & 372.9 & 3 & 433.2 \\
\hline 574.3 & 577.6 & 3 & 550.2 \\
\hline 695 & 697.7 & 3 & 171.1 \\
\hline 472.6 & 475.3 & 3 & 635.4 \\
\hline 834.4 & 838.4 & 2 & 228.1 \\
\hline 660.8 & 664.8 & 2 & 976.5 \\
\hline 469.7 & 473.7 & 2 & 504.2 \\
\hline 470.2 & 475.2 & 2 & 536.3 \\
\hline 571.9 & 575.2 & 3 & 405.7 \\
\hline 447.2 & 452.2 & 2 & 622.3 \\
\hline 469.7 & 473.7 & 2 & 723.3 \\
\hline 613.3 & 618.3 & 2 & 750.4 \\
\hline 462.3 & 467.3 & 2 & 696.4 \\
\hline
\end{tabular}

\begin{tabular}{|c|c|}
\hline & 841.4 \\
\hline & 829.4 \\
\hline & 593.3 \\
\hline 501.2 & 282.2 \\
\hline & 569.3 \\
\hline & 821.5 \\
\hline & 886.4 \\
\hline & 322.1 \\
\hline & 754.4 \\
\hline & 720.5 \\
\hline & 932.5 \\
\hline & 438.2 \\
\hline & 560.2 \\
\hline 797.4 & 171.1 \\
\hline & 635.4 \\
\hline 433.2 & 228.1 \\
\hline & 984.5 \\
\hline & 512.3 \\
\hline & 546.3 \\
\hline & 410.7 \\
\hline & 632.3 \\
\hline & 731.4 \\
\hline & 760.4 \\
\hline & 706.4 \\
\hline
\end{tabular}

\begin{tabular}{|c|c|c|c|}
\hline y7 & & $Y$ & 21.3 \\
\hline y8 & & $Y$ & 21.4 \\
\hline y10 & & $Y$ & 23.7 \\
\hline y2 & b4 & $Y$ & 17.9 \\
\hline y5 & & $Y$ & 15.2 \\
\hline y8 & & $Y$ & 17 \\
\hline y7 & & $Y$ & 18.9 \\
\hline b3 & & $Y$ & 27.2 \\
\hline y7 & & $Y$ & 14.3 \\
\hline y6 & & $Y$ & 21.6 \\
\hline y8 & & $Y$ & 28.4 \\
\hline y7 & & $Y$ & 8.5 \\
\hline y4 & & $Y$ & 15.9 \\
\hline b2 & y8 & $Y$ & 20.2 \\
\hline b6 & & $Y$ & 12.2 \\
\hline b2 & y5 & $Y$ & 26.9 \\
\hline y9 & & $Y$ & 21.5 \\
\hline y5 & & $Y$ & 15.6 \\
\hline y4 & & $Y$ & 15.6 \\
\hline y8 & & $Y$ & 15.8 \\
\hline y6 & & $Y$ & 14.9 \\
\hline y5 & & $Y$ & 15.6 \\
\hline y6 & & $Y$ & 20 \\
\hline y6 & & $Y$ & 15.3 \\
\hline
\end{tabular}




\begin{tabular}{|l|l|l|} 
P03951 & FA11 & SCALSNLACIR \\
\hline P03951 & FA11 & TAAISGYSFK \\
\hline P03951 & FA11 & VVSGFSLK \\
\hline P00748 & FA12 & CFEPQLLR \\
\hline P00748 & FA12 & LHEAFSPVSYQHDLALLR \\
\hline P00748 & FA12 & NGPLSCGQR \\
\hline P00748 & FA12 & NWGLGGHAFCR \\
\hline P00748 & FA12 & TEQAAVAR \\
\hline P00748 & FA12 & VVGGLVALR \\
\hline P12259 & FA5 & AVQPGETYTYK \\
\hline P12259 & FA5 & EFNPLVIVGLSK \\
\hline P12259 & FA5 & EVIITGIQTQGAK \\
\hline P12259 & FA5 & GEYEEHLGILGPIIR \\
\hline P12259 & FA5 & LAAALGIR \\
\hline P12259 & FA5 & NFFNPPIISR \\
\hline P08709 & FA7 & LHQPVVLTDHVVPLCLPER \\
\hline P00740 & FA9 & NCELDVTCNIK \\
\hline P00740 & FA9 & SALVLQYLR \\
\hline P00740 & FA9 & SCEPAVPFPCGR \\
\hline P00740 & FA9 & VSVSQTSK \\
\hline P00740 & FA9 & VVCSCTEGYR \\
\hline P23142 & FBLN1 & CVDVDECAPPAEPCGK \\
\hline P23142 & FBLN1 & SQETGDLDVGGLQETDK \\
\hline P23142 & FBLN1 & TGYYFDGISR \\
\hline
\end{tabular}

\begin{tabular}{|c|c|c|c|}
\hline 632.8 & 637.8 & 2 & 319.1 \\
\hline 522.8 & 526.8 & 2 & 688.3 \\
\hline 418.7 & 422.8 & 2 & 638.4 \\
\hline 531.8 & 536.8 & 2 & 626.4 \\
\hline 699.4 & 702.7 & 3 & 251.2 \\
\hline 494.7 & 499.7 & 2 & 409.2 \\
\hline 425.5 & 428.9 & 3 & 487.7 \\
\hline 423.2 & 428.2 & 2 & 615.4 \\
\hline 442.3 & 447.3 & 2 & 685.4 \\
\hline 628.8 & 632.8 & 2 & 299.2 \\
\hline 658.4 & 662.4 & 2 & 925.6 \\
\hline 679.4 & 683.4 & 2 & 903.5 \\
\hline 566 & 569.3 & 3 & 668.4 \\
\hline 392.8 & 397.8 & 2 & 600.4 \\
\hline 602.8 & 607.8 & 2 & 796.5 \\
\hline 741.4 & 744.7 & 3 & 251.2 \\
\hline 683.3 & 687.3 & 2 & 275.1 \\
\hline 531.8 & 536.8 & 2 & 692.4 \\
\hline 688.8 & 693.8 & 2 & 1000.5 \\
\hline 418.2 & 422.2 & 2 & 550.3 \\
\hline 615.8 & 620.8 & 2 & 1032.4 \\
\hline 902.4 & 906.4 & 2 & 855.4 \\
\hline 896.4 & 900.4 & 2 & 847.4 \\
\hline 589.8 & 594.8 & 2 & 694.4 \\
\hline
\end{tabular}

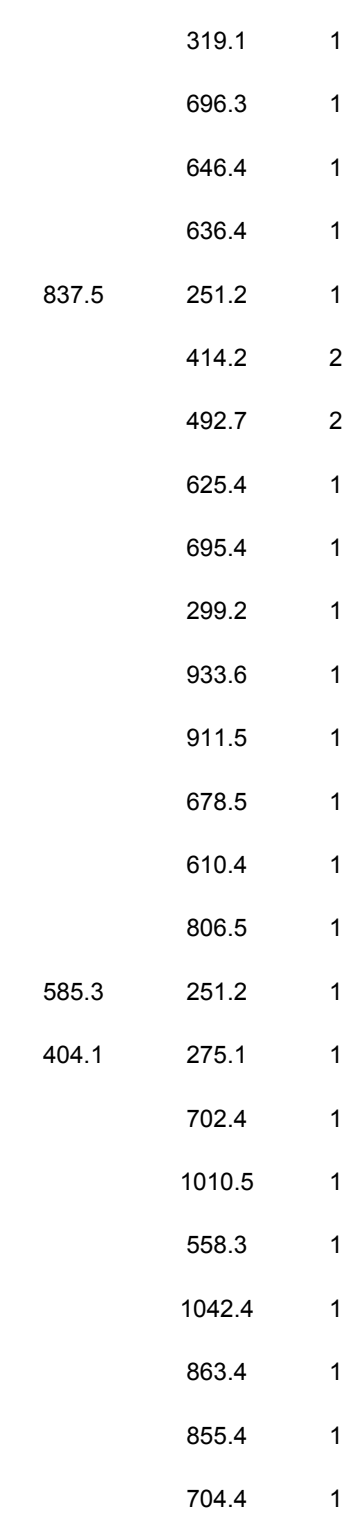

\begin{tabular}{|c|c|c|c|}
\hline b3 & & $Y$ & 20.6 \\
\hline y6 & & $Y$ & 17.2 \\
\hline y6 & & $Y$ & 14 \\
\hline y5 & & $Y$ & 17.5 \\
\hline b2 & y7 & $Y$ & 20.4 \\
\hline y7 & & $Y$ & 16.3 \\
\hline y9 & & $Y$ & 10.5 \\
\hline y6 & & $Y$ & 14.1 \\
\hline y7 & & $Y$ & 14.7 \\
\hline b3 & & $Y$ & 20.5 \\
\hline y9 & & $Y$ & 21.4 \\
\hline y9 & & $Y$ & 22.1 \\
\hline y6 & & $Y$ & 15.6 \\
\hline y6 & & $Y$ & 13.2 \\
\hline y7 & & $Y$ & 19.7 \\
\hline b2 & b5 & $Y$ & 21.9 \\
\hline b2 & b3 & $Y$ & 22.2 \\
\hline y5 & & $Y$ & 17.5 \\
\hline y9 & & $Y$ & 22.4 \\
\hline y5 & & $Y$ & 14 \\
\hline y8 & & $Y$ & 20.1 \\
\hline y8 & & $Y$ & 29 \\
\hline y8 & & $Y$ & 28.8 \\
\hline y6 & & $Y$ & 19.3 \\
\hline
\end{tabular}




\begin{tabular}{|l|l|l|} 
Q12805 & FBLN3 & NPCQDPYILTPENR \\
\hline Q12805 & FBLN3 & SGNENGEFYLR \\
\hline P35556 & FBN2 & FNLSHLGSK \\
\hline P22087 & FBRL & NGGHFVISIK \\
\hline P08637 & FCG3A & AVVFLEPQWYR \\
\hline Q9Y6R7 & FCGBP & AIGYATAADCGR \\
\hline Q9Y6R7 & FCGBP & LASVSVSR \\
\hline Q9Y6R7 & FCGBP & VNGVLTALPVSVADGR \\
\hline O75636 & FCN3 & YAVSEAAAHK \\
\hline O75636 & FCN3 & YGIDWASGR \\
\hline P02765 & FETUA & CNLLAEK \\
\hline P02765 & FETUA & EATEAAK \\
\hline P02765 & FETUA & EHAVEGDCDFQLLK \\
\hline P02765 & FETUA & FSVVYAK \\
\hline P02765 & FETUA & TVVQPSVGAAAGPVVPCPG \\
\hline P02765 & FETUA & VVHAAK \\
\hline Q9UGM5 & FETUB & DGYVLR \\
\hline Q9UGM5 & FETUB & SQASSCSLQSSDSVPVGLCK \\
\hline Q9UGM5 & FETUB & VNDAQEYR \\
\hline Q03591 & FHR1 & STDTSCVNPPTVQNAHILSR \\
\hline Q03591 & FHR1 & TGESAEFVCK \\
\hline Q02985 & FHR3 & AQTTVTCTEK \\
\hline Q9BXR6 & FHR5 & IAGVNIK \\
\hline Q9BXR6 & FHR5 & LQGSVTVTCR \\
\hline
\end{tabular}

$\begin{array}{llll}858.9 & 863.9 & 2 & 515.3 \\ 643.3 & 648.3 & 2 & 784.4 \\ 501.8 & 505.8 & 2 & 262.1 \\ 536.3 & 540.3 & 2 & 706.4 \\ 704.4 & 709.4 & 2 & 749.4 \\ 613.3 & 618.3 & 2 & 821.4 \\ 409.7 & 414.7 & 2 & 634.4 \\ 784.4 & 789.4 & 2 & 913.5 \\ 349.5 & 352.2 & 3 & 235.1 \\ 512.7 & 517.8 & 2 & 691.3 \\ 424.2 & 428.2 & 2 & 573.4 \\ 360.2 & 364.2 & 2 & 289.2 \\ 554.3 & 556.9 & 3 & 147.1 \\ 407.2 & 411.2 & 2 & 666.4 \\ 672.7 & 676 & 3 & 342.2 \\ 312.7 & 316.7 & 2 & 426.2 \\ 361.7 & 366.7 & 2 & 387.3 \\ 1049 & 1053 & 2 & 673.4 \\ 497.7 & 502.7 & 2 & 781.3 \\ 733 & 736.4 & 3 & 618.3 \\ 564.3 & 568.3 & 2 & 840.4 \\ 569.8 & 573.8 & 2 & 200.1 \\ 357.7 & 361.7 & 2 & 601.4 \\ 560.8 & 565.8 & 2 & 879.4\end{array}$

\begin{tabular}{|c|c|c|}
\hline & 525.3 & 1 \\
\hline & 794.4 & 1 \\
\hline \multirow[t]{6}{*}{628.3} & 262.1 & 1 \\
\hline & 714.5 & 1 \\
\hline & 759.4 & 1 \\
\hline & 831.4 & 1 \\
\hline & 644.4 & 1 \\
\hline & 923.5 & 1 \\
\hline \multirow[t]{13}{*}{497.3} & 235.1 & 1 \\
\hline & 701.3 & 1 \\
\hline & 581.4 & 1 \\
\hline & 297.2 & 1 \\
\hline & 155.1 & 1 \\
\hline & 674.4 & 1 \\
\hline & 347.2 & 2 \\
\hline & 434.3 & 1 \\
\hline & 397.3 & 1 \\
\hline & 681.4 & 1 \\
\hline & 791.4 & 1 \\
\hline & 623.4 & 2 \\
\hline & 848.4 & 1 \\
\hline \multirow[t]{3}{*}{638.3} & 200.1 & 1 \\
\hline & 609.4 & 1 \\
\hline & 889.4 & 1 \\
\hline
\end{tabular}

ty6




\begin{tabular}{|l|l|l|} 
Q9BXR6 & FHR5 & TGDAVEFQCK \\
\hline P02671 & FIBA & DLLPSR \\
\hline P02671 & FIBA & DNTYNR \\
\hline P02671 & FIBA & ESSSHHPGIAEFPSR \\
\hline P02671 & FIBA & GDFSSANNR \\
\hline P02671 & FIBA & GLIDEVNQDFTNR \\
\hline P02671 & FIBA & GSESGIFTNTK \\
\hline P02671 & FIBA & VQHIQLLQK \\
\hline P02675 & FIBB & DNENVVNEYSSELEK \\
\hline P02675 & FIBB & HQLYIDETVNSNIPTNLR \\
\hline P02675 & FIBB & NYCGLPGEYWLGNDK \\
\hline P02675 & FIBB & QGFGNVATNTDGK \\
\hline P02675 & FIBB & SILENLR \\
\hline P02679 & FIBG & ASTPNGYDNGIIWATWK \\
\hline P02679 & FIBG & DNCCILDER \\
\hline P02679 & FIBG & EGFGHLSPTGTTEFWLGNEK \\
\hline P02679 & FIBG & VELEDWNGR \\
\hline P02679 & FIBG & YEASILTHDSSIR \\
\hline P02679 & FIBG & YLQEIYNSNNQK \\
\hline P02751 & FINC & DLQFVEVTDVK \\
\hline P02751 & FINC & FLATTPNSLLVSWQPPR \\
\hline P02751 & FINC & GEWTCIAYSQLR \\
\hline P02751 & FINC & HTSVQTTSSGSGPFTDVR \\
\hline P02751 & FINC & IGDTWR \\
\hline
\end{tabular}

\begin{tabular}{|c|c|c|c|}
\hline 577.8 & 581.8 & 2 & 274.1 \\
\hline 350.7 & 355.7 & 2 & 359.2 \\
\hline 391.7 & 396.7 & 2 & 553.3 \\
\hline 819.4 & 824.4 & 2 & 973.5 \\
\hline 484.2 & 489.2 & 2 & 648.3 \\
\hline 760.9 & 765.9 & 2 & 894.4 \\
\hline 570.8 & 574.8 & 2 & 610.3 \\
\hline 553.8 & 557.8 & 2 & 879.5 \\
\hline 884.9 & 888.9 & 2 & 572.2 \\
\hline 1064 & 1069 & 2 & 600.3 \\
\hline 893.4 & 897.4 & 2 & 1178.5 \\
\hline 654.8 & 658.8 & 2 & 706.3 \\
\hline 422.7 & 427.8 & 2 & 644.4 \\
\hline 947.5 & 951.5 & 2 & 804.4 \\
\hline 597.7 & 602.8 & 2 & 532.3 \\
\hline 736.4 & 739 & 3 & 447.2 \\
\hline 559.3 & 564.3 & 2 & 229.1 \\
\hline 746.4 & 751.4 & 2 & 815.4 \\
\hline 757.4 & 761.4 & 2 & 867.4 \\
\hline 646.8 & 650.8 & 2 & 789.4 \\
\hline 964 & 969 & 2 & 369.2 \\
\hline 742.4 & 747.4 & 2 & 503.3 \\
\hline 622 & 625.3 & 3 & 734.4 \\
\hline 374.2 & 379.2 & 2 & 634.3 \\
\hline
\end{tabular}

\begin{tabular}{ll}
274.1 & 1 \\
369.2 & 1 \\
563.3 & 1 \\
983.5 & 1 \\
658.3 & 1 \\
904.4 & 1 \\
618.3 & 1 \\
\hline 887.6 & 1 \\
572.2 & 1 \\
610.4 & 1 \\
1186.6 & 1 \\
\hline 714.4 & 1 \\
\hline 654.4 & 1 \\
\hline 812.5 & 1 \\
\hline 542.3 & 1 \\
\hline 455.2 & 1 \\
\hline 229.1 & 1 \\
825.4 & 1 \\
875.4 & 1 \\
797.4 & 1 \\
379.2 & 1 \\
513.3 & 1 \\
744.4 & 1 \\
644.3 & 1
\end{tabular}

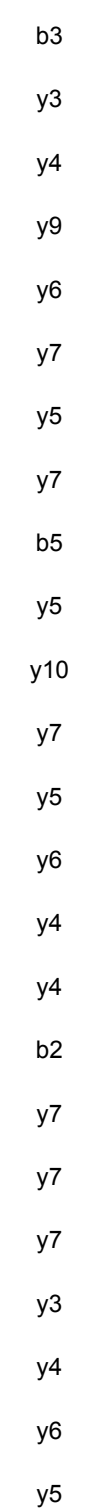

\begin{tabular}{l|}
18.9 \\
11.9 \\
13.1 \\
26.4 \\
16 \\
24.6 \\
18.7 \\
18.2 \\
28.4 \\
34 \\
28.7 \\
21.3 \\
14.1 \\
30.4 \\
19.5 \\
21.7 \\
18.3 \\
24.1 \\
24.5 \\
21.1 \\
30.9 \\
24 \\
17.6 \\
12.6 \\
\hline
\end{tabular}




\begin{tabular}{|l|l|l|} 
P02751 & FINC & ISCTIANR \\
\hline P02751 & FINC & IYLYTLNDNAR \\
\hline P02751 & FINC & LLCQCLGFGSGHFR \\
\hline P02751 & FINC & LTVGLTR \\
\hline P02751 & FINC & QDGHLWCSTTSNYEQDQK \\
\hline P02751 & FINC & QYNVGPSVSK \\
\hline P02751 & FINC & SSPVVIDASTAIDAPSNLR \\
\hline P02751 & FINC & SYTITGLQPGTDYK \\
\hline P02751 & FINC & TYLGNALVCTCYGGSR \\
\hline P02751 & FINC & VPGTSTSATLTGLTR \\
\hline P02751 & FINC & YSFCTDHTVLVQTR \\
\hline Q06787 & FMR1 & EPCCWWLAK \\
\hline O95954 & FTCD & SDLQVAAK \\
\hline P06396 & GELS & EVQGFESATFLGYFK \\
\hline P06396 & GELS & HVVPNEVVVQR \\
\hline P06396 & GELS & QTQVSVLPEGGETPLFK \\
\hline P06396 & GELS & SEDCFILDHGK \\
\hline P06396 & GELS & TGAQELLR \\
\hline P06396 & GELS & TPSAAYLWVGTGASEAEK \\
\hline P06396 & GELS & YIETDPANR \\
\hline Q92820 & GGH & YLESAGAR \\
\hline P22352 & GPX3 & FYTFLK \\
\hline P22352 & GPX3 & NSCPPTSELLGTSDR \\
\hline P22352 & GPX3 & TTVSNVK \\
\hline
\end{tabular}

\begin{tabular}{l}
467.7 \\
678.4 \\
551.3 \\
380.2 \\
733 \\
539.8 \\
957 \\
772.4 \\
896.4 \\
731.4 \\
576.3 \\
625.3 \\
416.2 \\
861.9 \\
425.9 \\
915.5 \\
660.8 \\
444.3 \\
919.5 \\
\hline 539.8 \\
\hline 374.7 \\
\hline
\end{tabular}

$\begin{array}{lll}472.7 & 2 & 734.4 \\ 683.4 & 2 & 277.2 \\ 554.6 & 3 & 660.3 \\ 385.2 & 2 & 545.3 \\ 735.7 & 3 & 810.4 \\ 543.8 & 2 & 574.3 \\ 962 & 2 & 586.3 \\ 776.4 & 2 & 680.3 \\ 901.4 & 2 & 960.4 \\ 736.4 & 2 & 761.5 \\ 579.6 & 3 & 782.4 \\ 629.3 & 2 & 218.1 \\ 420.2 & 2 & 629.4 \\ 865.9 & 2 & 875.5 \\ 429.2 & 3 & 501.3 \\ 919.5 & 2 & 1074.5 \\ 664.8 & 2 & 569.3 \\ 449.3 & 2 & 159.1 \\ 923.5 & 2 & 849.4 \\ 544.8 & 2 & 802.4 \\ 4338.7 & 2 & 590.3 \\ 413.7 & 2 & 508.3 \\ 822.4 & 2 & 636.8 \\ 378.7 & 2 & 203.1\end{array}$

\begin{tabular}{|}
803.4 \\
\hline 863.4 \\
\hline 546.3 \\
\hline
\end{tabular}

$670.3 \quad 1$

$555.3 \quad 1$

$818.4 \quad 1$

$582.3 \quad 1$

$596.3 \quad 1$

$688.3 \quad 1$

$970.4 \quad 1$

$771.5 \quad 1$

$\begin{array}{ll}787.4 & 2\end{array}$

$\begin{array}{lll}62.4 & 226.2 & 1\end{array}$

$637.4 \quad 1$

$883.5 \quad 1$

$511.3 \quad 1$

$1082.6 \quad 1$

$577.3 \quad 1$

$159.1 \quad 1$

$857.4 \quad 1$

$812.4 \quad 1$

$600.3 \quad 1$

$516.3 \quad 1$

$641.8 \quad 2$

$203.1 \quad 1$ 


\begin{tabular}{|l|l|l|} 
Q14520 & HABP2 & FCEIGSDDCYVGDGYSYR \\
\hline Q14520 & HABP2 & IYGGFK \\
\hline Q14520 & HABP2 & LIANTLCNSR \\
\hline Q14520 & HABP2 & LPGFDSCGK \\
\hline P69905 & HBA & VGAHAGEYGAEALER \\
\hline P08397 & HEM3 & ELEHALEK \\
\hline P02790 & HEMO & ELISER \\
\hline P02790 & HEMO & GGYTLVSGYPK \\
\hline P02790 & HEMO & LLQDEFPGIPSPLDAAVECHR \\
\hline P02790 & HEMO & LYLVQGTQVYVFLTK \\
\hline P02790 & HEMO & NFPSPVDAAFR \\
\hline P02790 & HEMO & SGAQATWTELPWPHEK \\
\hline P02790 & HEMO & YYCFQGNQFLR \\
\hline P05546 & HEP2 & NFGYTLR \\
\hline P05546 & HEP2 & NYNLVESLK \\
\hline P05546 & HEP2 & QFPILLDFK \\
\hline P05546 & HEP2 & SVNDLYIQK \\
\hline P05546 & HEP2 & TLEAQLTPR \\
\hline Q04756 & HGFA & LCNIEPDER \\
\hline Q04756 & HGFA & LEACESLTR \\
\hline Q04756 & HGFA & SQFVQPICLPEPGSTFPAGHK \\
\hline Q04756 & HGFA & TTDVTQTFGIEK \\
\hline Q04756 & HGFA & VANYVDWINDR \\
\hline P00738 & HPT & VGYVSGWGR \\
\hline
\end{tabular}

\begin{tabular}{llll}
1081.9 & 1086.9 & 2 & 817.3 \\
342.7 & 346.7 & 2 & 408.2 \\
581.3 & 586.3 & 2 & 1048.5 \\
490.7 & 494.7 & 2 & 434.2 \\
510.6 & 513.9 & 3 & 617.3 \\
484.8 & 488.8 & 2 & 726.4 \\
373.7 & 378.7 & 2 & 391.2 \\
571.3 & 575.3 & 2 & 650.4 \\
788.7 & 792.1 & 3 & 676.3 \\
886.5 & 890.5 & 2 & 277.2 \\
610.8 & 615.8 & 2 & 775.4 \\
613.3 & 616 & 3 & 793.4 \\
748.3 & 753.3 & 2 & 862.5 \\
435.7 & 440.7 & 2 & 609.3 \\
540.3 & 544.3 & 2 & 802.5 \\
560.8 & 564.8 & 2 & 845.5 \\
540.3 & 544.3 & 2 & 893.5 \\
514.8 & 519.8 & 2 & 814.4 \\
573.3 & 578.3 & 2 & 872.4 \\
539.8 & 544.8 & 2 & 836.4 \\
766.4 & 769.1 & 3 & 216.1 \\
670.3 & 674.3 & 2 & 923.5 \\
682.8 & 687.8 & 2 & 818.4 \\
490.8 & 495.8 & 2 & 562.3 \\
\hline 50.3 \\
\hline 50.3 \\
\hline 50.3
\end{tabular}

\begin{tabular}{|c|c|c|}
\hline & 827.4 & 1 \\
\hline & 416.2 & 1 \\
\hline & 1058.5 & 1 \\
\hline & 438.2 & 2 \\
\hline & 627.3 & 1 \\
\hline & 734.4 & 1 \\
\hline & 401.2 & 1 \\
\hline & 658.4 & 1 \\
\hline & 681.3 & 2 \\
\hline \multirow[t]{11}{*}{390.2} & 277.2 & 1 \\
\hline & 785.4 & 1 \\
\hline & 801.4 & 1 \\
\hline & 872.5 & 1 \\
\hline & 619.3 & 1 \\
\hline & 810.5 & 1 \\
\hline & 853.5 & 1 \\
\hline & 901.5 & 1 \\
\hline & 824.5 & 1 \\
\hline & 882.4 & 1 \\
\hline & 846.4 & 1 \\
\hline \multirow[t]{4}{*}{499.8} & 216.1 & 1 \\
\hline & 931.5 & 1 \\
\hline & 828.4 & 1 \\
\hline & 572.3 & 1 \\
\hline
\end{tabular}




\begin{tabular}{|l|l|l|} 
P00738 & HPT & VSVNER \\
\hline P00738 & HPT & VTSIQDWVQK \\
\hline P00739 & HPTR & VVLHPNYHQVDIGLIK \\
\hline P04196 & HRG & ADLFYDVEALDLESPK \\
\hline P04196 & HRG & DGYLFQLLR \\
\hline P04196 & HRG & GGEGTGYFVDFSVR \\
\hline P04196 & HRG & QIGSVYR \\
\hline P04196 & HRG & YWNDCEPPDSR \\
\hline P18065 & IBP2 & GECWCVNPNTGK \\
\hline P18065 & IBP2 & LIQGAPTIR \\
\hline P17936 & IBP3 & ALAQCAPPPAVCAELVR \\
\hline P17936 & IBP3 & ETEYGPCR \\
\hline P17936 & IBP3 & FLNVLSPR \\
\hline P17936 & IBP3 & YGQPLPGYTTK \\
\hline P24593 & IBP5 & AVYLPNCDR \\
\hline P24593 & IBP5 & GVCLNEK \\
\hline P05155 & IC1 & FQPTLLTLPR \\
\hline P05155 & IC1 & GVTSVSQIFHSPDLAIR \\
\hline P05155 & IC1 & LLDSLPSDTR \\
\hline P05155 & IC1 & TLYSSSPR \\
\hline P05155 & IC1 & TNLESILSYPK \\
\hline P05155 & IC1 & TTFDPK \\
\hline P05362 & ICAM1 & VELAPLPSWQPVGK \\
\hline P22304 & IDS & QSTEQAIQLLEK \\
\hline
\end{tabular}

\begin{tabular}{|c|c|c|c|}
\hline 352.2 & 357.2 & 2 & 604.3 \\
\hline 602.3 & 606.3 & 2 & 1003.5 \\
\hline 615.7 & 618.4 & 3 & 698.9 \\
\hline 609 & 611.6 & 3 & 688.4 \\
\hline 562.8 & 567.8 & 2 & 676.4 \\
\hline 745.8 & 750.9 & 2 & 869.5 \\
\hline 411.7 & 416.7 & 2 & 581.3 \\
\hline 719.8 & 724.8 & 2 & 571.3 \\
\hline 711.3 & 715.3 & 2 & 516.3 \\
\hline 484.8 & 489.8 & 2 & 742.4 \\
\hline 912 & 917 & 2 & 1208.6 \\
\hline 506.2 & 511.2 & 2 & 489.2 \\
\hline 473.3 & 478.3 & 2 & 685.4 \\
\hline 612.8 & 616.8 & 2 & 876.5 \\
\hline 554.3 & 559.3 & 2 & 661.3 \\
\hline 410.2 & 414.2 & 2 & 663.3 \\
\hline 395.9 & 399.2 & 3 & 486.3 \\
\hline 914 & 919 & 2 & 771.4 \\
\hline 558.8 & 563.8 & 2 & 575.3 \\
\hline 455.7 & 460.7 & 2 & 696.3 \\
\hline 632.8 & 636.8 & 2 & 216.1 \\
\hline 354.7 & 358.7 & 2 & 244.2 \\
\hline 760.9 & 764.9 & 2 & 342.2 \\
\hline 694.4 & 698.4 & 2 & 814.5 \\
\hline
\end{tabular}

\begin{tabular}{ll}
614.3 & 1 \\
1011.5 & 1 \\
702.9 & 2 \\
696.4 & 1 \\
686.4 & 1 \\
879.5 & 1 \\
591.3 & 1 \\
\hline 581.3 & 1 \\
524.3 & 1 \\
752.4 & 1 \\
1218.7 & 1 \\
499.2 & 1 \\
\hline 695.4 & 1 \\
\hline 884.5 & 1 \\
671.3 & 1 \\
671.3 & 1 \\
496.3 & 1 \\
781.4 & 1 \\
\hline 585.3 & 1 \\
706.3 & 1 \\
\hline 216.1 & 1 \\
252.2 & 1 \\
342.2 & 1 \\
822.5 & 1
\end{tabular}

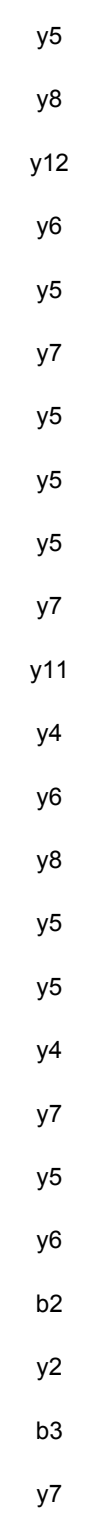

\begin{tabular}{c|c|}
11.9 & 19.7 \\
17.4 & 17.1 \\
18.4 & 24.1 \\
13.8 \\
23.3 \\
23.1 \\
16 \\
29.3 \\
16.7 \\
15.7 \\
20 \\
18.2 \\
13.7 \\
9.5 \\
29.3 \\
18.3 \\
15.1 \\
20.6 \\
12 \\
22.5 \\
\hline
\end{tabular}




\begin{tabular}{|l|l|l|} 
P01344 & IGF2 & GIVEECCFR \\
\hline P01344 & IGF2 & SCDLALLETYCATPAK \\
\hline P01857 & IGHG1 & TPEVTCVVVDVHEDPEVK \\
\hline P01860 & IGHG3 & SCDTPPPCPR \\
\hline P01871 & IGHM & QIQVSWLR \\
\hline P01871 & IGHM & QVGSGVTTDQVQAEAK \\
\hline P01871 & IGHM & YAATSQVLLPSK \\
\hline P01834 & IGKC & DSTYSLSSTLTLSK \\
\hline P01834 & IGKC & VDNALQSGNSQESVTEQDSK \\
\hline P05113 & IL5 & ETLALLSTHR \\
\hline P06213 & INSR & VCHLLEGEK \\
\hline P05154 & IPSP & AAAATGTIFTFR \\
\hline P05154 & IPSP & AVVEVDESGTR \\
\hline P05154 & IPSP & DFTFDLYR \\
\hline P05154 & IPSP & FSIEGSYQLEK \\
\hline P05154 & IPSP & TLYLADTFPTNFR \\
\hline P08514 & ITA2B & IVLLDVPVR \\
\hline P19827 & ITIH1 & AAISGENAGLVR \\
\hline P19827 & ITIH1 & FAHYVVTSQVVNTANEAR \\
\hline P19827 & ITIH1 & GSLVQASEANLQAAQDFVR \\
\hline P19827 & ITIH1 & QAVDTAVDGVFIR \\
\hline P19827 & ITIH1 & QLVHHFEIDVDIFEPQGISK \\
\hline P19827 & ITIH1 & QYYEGSEIVVAGR \\
\hline P19823 & ITIH2 & AEDHFSVIDFNQNIR \\
\hline
\end{tabular}

\begin{tabular}{|c|c|c|c|}
\hline 585.3 & 590.3 & 2 & 900.3 \\
\hline 906.9 & 910.9 & 2 & 315.2 \\
\hline 713.7 & 716.4 & 3 & 472.3 \\
\hline 593.8 & 598.8 & 2 & 723.4 \\
\hline 515.3 & 520.3 & 2 & 788.4 \\
\hline 809.4 & 813.4 & 2 & 1090.5 \\
\hline 639.4 & 643.4 & 2 & 331.2 \\
\hline 751.9 & 755.9 & 2 & 836.5 \\
\hline 1068.5 & 1072.5 & 2 & 707.3 \\
\hline 570.8 & 575.8 & 2 & 613.3 \\
\hline 542.8 & 546.8 & 2 & 413.2 \\
\hline 613.8 & 618.8 & 2 & 214.1 \\
\hline 581.3 & 586.3 & 2 & 763.4 \\
\hline 538.8 & 543.8 & 2 & 814.4 \\
\hline 650.8 & 654.8 & 2 & 953.5 \\
\hline 779.9 & 784.9 & 2 & 634.3 \\
\hline 512.3 & 517.3 & 2 & 811.5 \\
\hline 579.3 & 584.3 & 2 & 902.5 \\
\hline 669.3 & 672.7 & 3 & 775.4 \\
\hline 1002.5 & 1007.5 & 2 & 806.4 \\
\hline 695.9 & 700.9 & 2 & 706.4 \\
\hline 784.4 & 787.1 & 3 & 667.3 \\
\hline 735.9 & 740.9 & 2 & 887.5 \\
\hline 902.9 & 907.9 & 2 & 791.4 \\
\hline
\end{tabular}

\begin{tabular}{ll}
910.3 & 1 \\
323.2 & 1 \\
480.3 & 1 \\
733.4 & 1 \\
798.4 & 1 \\
1098.6 & 1 \\
339.2 & 1 \\
844.5 & 1 \\
715.3 & 1 \\
623.3 & 1 \\
4117.2 & 2 \\
\hline 214.1 & 1 \\
\hline 773.4 & 1 \\
824.4 & 1 \\
\hline 961.5 & 1 \\
\hline 644.3 & 1 \\
821.5 & 1 \\
\hline 912.5 & 1 \\
785.4 & 1 \\
816.4 & 1 \\
716.4 & 1 \\
667.3 & 2 \\
897.5 & 1 \\
801.4 & 1
\end{tabular}




\begin{tabular}{|l|l|l|} 
P19823 & ITIH2 & IYLQPGR \\
\hline P19823 & ITIH2 & TEVNVLPGAK \\
\hline P19823 & ITIH2 & TQVADAK \\
\hline P19823 & ITIH2 & VVNNSPQPQNVVFDVQIPK \\
\hline Q06033 & ITIH3 & EHLVQATPENLQEAR \\
\hline Q06033 & ITIH3 & EVSFDVELPK \\
\hline Q06033 & ITIH3 & SLPEGVANGIEVYSTK \\
\hline Q14624 & ITIH4 & GPDVLTATVSGK \\
\hline Q14624 & ITIH4 & ILDDLSPR \\
\hline Q14624 & ITIH4 & LALDNGGLAR \\
\hline Q14624 & ITIH4 & NPLVWVHASPEHVVVTR \\
\hline Q14624 & ITIH4 & NVVFVIDK \\
\hline O60674 & JAK2 & SDNIIFQFTK \\
\hline P35527 & K1C9 & FSSSGGGGGGGR \\
\hline P35527 & K1C9 & FSSSSGYGGGSSR \\
\hline P35527 & K1C9 & TLLDIDNTR \\
\hline P35527 & K1C9 & VQALEEANNDLENK \\
\hline P04264 & K2C1 & SLDLDSIIAEVK \\
\hline P04264 & K2C1 & SLVNLGGSK \\
\hline P04264 & K2C1 & TLLEGEESR \\
\hline P13647 & K2C5 & ISISTSGGSFR \\
\hline P29622 & KAIN & GDATVFFILPNQGK \\
\hline P29622 & KAIN & GFQHLLHTLNLPGHGLETR \\
\hline P29622 & KAIN & WADLSGITK \\
\hline
\end{tabular}

\begin{tabular}{|c|c|c|c|}
\hline 423.7 & 428.7 & 2 & 570.3 \\
\hline 514.3 & 518.3 & 2 & 231.1 \\
\hline 366.7 & 370.7 & 2 & 503.3 \\
\hline 1061.6 & 1065.6 & 2 & 244.2 \\
\hline 867.9 & 872.9 & 2 & 267.1 \\
\hline 581.8 & 585.8 & 2 & 700.4 \\
\hline 555.3 & 558 & 3 & 726.4 \\
\hline 572.8 & 576.8 & 2 & 663.4 \\
\hline 464.8 & 469.8 & 2 & 702.3 \\
\hline 500.3 & 505.3 & 2 & 815.4 \\
\hline 970.5 & 975.5 & 2 & 424.3 \\
\hline 467.3 & 471.3 & 2 & 720.4 \\
\hline 606.8 & 610.8 & 2 & 670.4 \\
\hline 491.7 & 496.7 & 2 & 748.3 \\
\hline 618.3 & 623.3 & 2 & 520.2 \\
\hline 530.8 & 535.8 & 2 & 215.1 \\
\hline 793.9 & 797.9 & 2 & 917.4 \\
\hline 651.9 & 655.9 & 2 & 874.5 \\
\hline 437.8 & 441.8 & 2 & 674.4 \\
\hline 517.3 & 522.3 & 2 & 819.4 \\
\hline 556.3 & 561.3 & 2 & 798.4 \\
\hline 753.9 & 757.9 & 2 & 543.3 \\
\hline 714.1 & 717.4 & 3 & 866.4 \\
\hline 495.8 & 499.8 & 2 & 733.4 \\
\hline
\end{tabular}

$\begin{array}{lll} & 580.3 & 1 \\ 698.4 & 231.1 & 1 \\ & 511.3 & 1 \\ 380.2 & 252.2 & 1 \\ & 267.1 & 1 \\ & 708.4 & 1 \\ & 734.4 & 1 \\ & 671.4 & 1 \\ & 712.3 & 1 \\ & 825.4 & 1 \\ & 424.3 & 1 \\ & 728.4 & 1 \\ & 678.4 & 1 \\ 733.3 & 758.3 & 1 \\ & 530.3 & 1 \\ & 215.1 & 1 \\ & 925.4 & 1 \\ & 882.5 & 1 \\ & 682.4 & 1 \\ & 829.4 & 1 \\ & 808.4 & 1 \\ & 551.3 & 1 \\ & 876.5 & 1 \\ & 741.4 & 1\end{array}$




\begin{tabular}{|l|l|l|} 
P06732 & KCRM & ELFDPIISDR \\
\hline O75037 & KI21B & AQEQGVAGPEFK \\
\hline P03952 & KLKB1 & FGCFLK \\
\hline P03952 & KLKB1 & IAYGTQGSSGYSLR \\
\hline P03952 & KLKB1 & LCNTGDNSVCTTK \\
\hline P03952 & KLKB1 & LVGITSWGEGCAR \\
\hline P03952 & KLKB1 & VNIPLVTNEECQK \\
\hline P01042 & KNG1 & AATGECTATVGK \\
\hline P01042 & KNG1 & ENFLFLTPDCK \\
\hline P01042 & KNG1 & QVVAGLNFR \\
\hline P01042 & KNG1 & TVGSDTFYSFK \\
\hline P01042 & KNG1 & YNSQNQSNNQFVLYR \\
\hline P05455 & LA & IGCLLK \\
\hline P11279 & LAMP1 & ALQATVGNSYK \\
\hline P13473 & LAMP2 & IPLNDLFR \\
\hline P18428 & LBP & GLQYAAQEGLLALQSELLR \\
\hline P18428 & LBP & ITLPDFTGDLR \\
\hline P18428 & LBP & LAEGFPLPLLK \\
\hline P18428 & LBP & VQLYDLGLQIHK \\
\hline P04180 & LCAT & LAGYLHTLVQNLVNNGYVR \\
\hline P04180 & LCAT & SSGLVSNAPGVQIR \\
\hline P04180 & LCAT & STELCGLWQGR \\
\hline P04180 & LCAT & TYSVEYLDSSK \\
\hline P07195 & LDHB & IVVVTAGVR \\
\hline
\end{tabular}

\begin{tabular}{|c|c|c|c|c|c|c|c|c|c|c|c|}
\hline 602.8 & 607.8 & 2 & 350.7 & & 355.7 & 2 & & y6 & & Y & 19.7 \\
\hline 630.8 & 634.8 & 2 & 294.2 & 648.3 & 302.2 & 1 & 1 & y2 & y6 & Y & 20.6 \\
\hline 386.2 & 390.2 & 2 & 624.3 & & 632.3 & 1 & & y5 & & $Y$ & 13 \\
\hline 730.4 & 735.4 & 2 & 826.4 & & 836.4 & 1 & & y8 & & $Y$ & 23.6 \\
\hline 735.3 & 739.3 & 2 & 509.2 & & 517.3 & 1 & & y4 & & $Y$ & 23.8 \\
\hline 703.3 & 708.4 & 2 & 1023.4 & & 1033.4 & 1 & & y9 & & $Y$ & 22.8 \\
\hline 772.4 & 776.4 & 2 & 214.1 & 908.4 & 214.1 & 1 & 1 & b2 & $y 7$ & $\mathrm{Y}$ & 24.9 \\
\hline 583.3 & 587.3 & 2 & 204.1 & 736.4 & 212.1 & 1 & 1 & y2 & y7 & Y & 19.1 \\
\hline 692.3 & 696.3 & 2 & 880.4 & & 888.4 & 1 & & y7 & & Y & 22.5 \\
\hline 502.3 & 507.3 & 2 & 677.4 & & 687.4 & 1 & & y6 & & Y & 16.6 \\
\hline 626.3 & 630.3 & 2 & 173.1 & & 173.1 & 2 & & b4 & & $Y$ & 20.4 \\
\hline 625.6 & 629 & 3 & 697.4 & & 707.4 & 1 & & y5 & & Y & 17.7 \\
\hline 352.2 & 356.2 & 2 & 590.3 & & 598.3 & 1 & & y5 & & Y & 11.9 \\
\hline 576.3 & 580.3 & 2 & 839.4 & & 847.4 & 1 & & y8 & & Y & 18.9 \\
\hline 494.3 & 499.3 & 2 & 777.4 & & 787.4 & 1 & & y6 & & Y & 16.3 \\
\hline 691.7 & 695.1 & 3 & 617.4 & & 627.4 & 1 & & y5 & & $\mathrm{Y}$ & 20.1 \\
\hline 624.3 & 629.3 & 2 & 215.1 & 460.3 & 215.1 & 1 & 1 & b2 & y4 & $\mathrm{Y}$ & 20.4 \\
\hline 599.4 & 603.4 & 2 & 680.5 & & 688.5 & 1 & & y6 & & $Y$ & 19.6 \\
\hline 476.3 & 478.9 & 3 & 228.1 & 695.4 & 228.1 & 1 & 1 & b2 & y6 & $Y$ & 12.3 \\
\hline 715.4 & 718.7 & 3 & 821.4 & & 831.4 & 1 & & y7 & & $\mathrm{Y}$ & 21 \\
\hline 692.9 & 697.9 & 2 & 669.4 & & 679.4 & 1 & & $\mathrm{y} 6$ & & $Y$ & 22.5 \\
\hline 653.8 & 658.8 & 2 & 876.4 & & 886.4 & 1 & & $y 7$ & & $Y$ & 21.3 \\
\hline 646.3 & 650.3 & 2 & 1027.5 & & 1035.5 & 1 & & y9 & & $Y$ & 21 \\
\hline 457.3 & 462.3 & 2 & 701.4 & & 711.4 & 1 & & y7 & & $Y$ & 15.2 \\
\hline
\end{tabular}




\begin{tabular}{|l|l|l|} 
P07195 & LDHB & SADTLWDIQK \\
\hline P51884 & LUM & FNALQYLR \\
\hline P51884 & LUM & ILGPLSYSK \\
\hline P51884 & LUM & ISNIPDEYFK \\
\hline P51884 & LUM & LPSGLPVSLLTLYLDNNK \\
\hline P51884 & LUM & NIPTVNENLENYYLEVNQLEK \\
\hline P51884 & LUM & SLEDLQLTHNK \\
\hline P14151 & LYAM1 & AEIEYLEK \\
\hline Q86UE4 & LYRIC & WNSVSPASAGK \\
\hline P61626 & LYSC & STDYGIFQINSR \\
\hline P61626 & LYSC & WESGYNTR \\
\hline P11226 & MBL2 & FQASVATPR \\
\hline P11226 & MBL2 & TEGQFVDLTGNR \\
\hline P11226 & MBL2 & WLTFSLGK \\
\hline O43772 & MCAT & EGITGLYR \\
\hline Q16674 & MIA & GQVVYVFSK \\
\hline P43246 & MSH2 & DIYQDLNR \\
\hline P61916 & NPC2 & SEYPSIK \\
\hline Q13093 & PAFA & ASLAFLQK \\
\hline P36955 & PEDF & ALYYDLISSPDIHGTYK \\
\hline P36955 & PEDF & ELLDTVTAPQK \\
\hline P36955 & PEDF & LQSLFDSPDFSK \\
\hline P36955 & PEDF & VLTGNPR \\
\hline P36955 & PEDF & YGLDSDLSCK \\
\hline
\end{tabular}

\begin{tabular}{|c|c|c|c|}
\hline 588.8 & 592.8 & 2 & 689.4 \\
\hline 512.8 & 517.8 & 2 & 763.4 \\
\hline 489.3 & 493.3 & 2 & 751.4 \\
\hline 613.3 & 617.3 & 2 & 798.4 \\
\hline 653 & 655.7 & 3 & 766.4 \\
\hline 1268.6 & 1272.6 & 2 & 631.3 \\
\hline 433.2 & 435.9 & 3 & 201.1 \\
\hline 497.8 & 501.8 & 2 & 794.4 \\
\hline 552.3 & 556.3 & 2 & 803.4 \\
\hline 700.8 & 705.8 & 2 & 764.4 \\
\hline 506.7 & 511.7 & 2 & 697.3 \\
\hline 488.8 & 493.8 & 2 & 701.4 \\
\hline 668.8 & 673.8 & 2 & 231.1 \\
\hline 476.3 & 480.3 & 2 & 765.5 \\
\hline 454.7 & 459.7 & 2 & 609.3 \\
\hline 513.8 & 517.8 & 2 & 186.1 \\
\hline 518.8 & 523.8 & 2 & 808.4 \\
\hline 412.2 & 416.2 & 2 & 607.3 \\
\hline 439.3 & 443.3 & 2 & 606.4 \\
\hline 652.7 & 655.3 & 3 & 185.1 \\
\hline 607.8 & 611.8 & 2 & 859.5 \\
\hline 692.3 & 696.4 & 2 & 242.1 \\
\hline 378.7 & 383.7 & 2 & 544.3 \\
\hline 579.3 & 583.3 & 2 & 824.3 \\
\hline
\end{tabular}

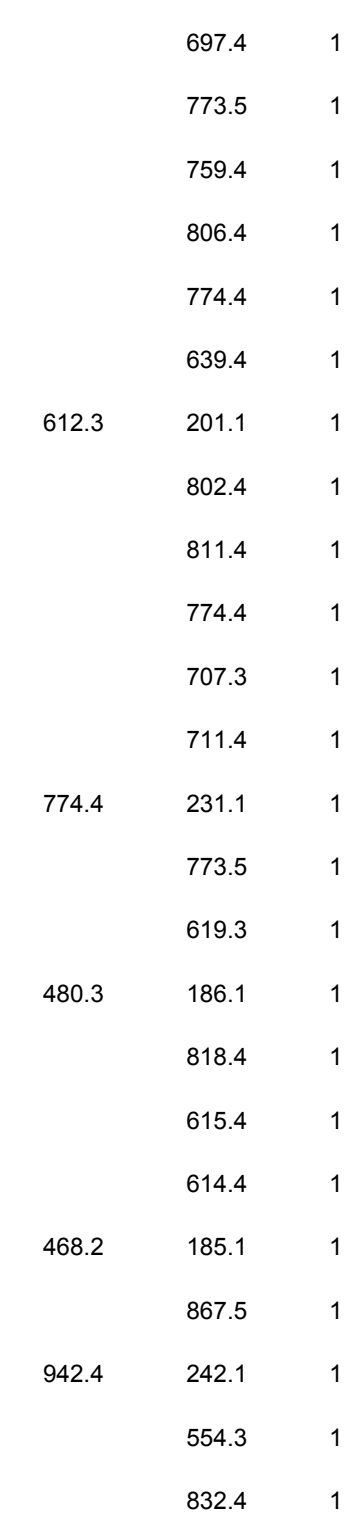

\begin{tabular}{|c|c|c|c|}
\hline y5 & & $Y$ & 19.3 \\
\hline y6 & & $Y$ & 16.9 \\
\hline y7 & & $Y$ & 16.2 \\
\hline y6 & & $Y$ & 20 \\
\hline y6 & & $Y$ & 18.7 \\
\hline y5 & & $Y$ & 40.3 \\
\hline b2 & y5 & $Y$ & 10.8 \\
\hline y6 & & $Y$ & 16.4 \\
\hline y9 & & $Y$ & 18.1 \\
\hline y6 & & $Y$ & 22.7 \\
\hline y6 & & $Y$ & 16.7 \\
\hline y7 & & $Y$ & 16.2 \\
\hline b2 & $y 7$ & $Y$ & 21.7 \\
\hline y7 & & $Y$ & 15.8 \\
\hline y5 & & $Y$ & 15.1 \\
\hline b2 & y4 & $Y$ & 16.9 \\
\hline y6 & & $Y$ & 17.1 \\
\hline y5 & & $Y$ & 13.8 \\
\hline y5 & & $Y$ & 14.6 \\
\hline b2 & y4 & $Y$ & 18.7 \\
\hline y8 & & $Y$ & 19.8 \\
\hline b2 & y8 & $Y$ & 22.5 \\
\hline y5 & & $Y$ & 12.7 \\
\hline y7 & & $Y$ & 19 \\
\hline
\end{tabular}




\begin{tabular}{|l|l|l|} 
P05164 & PERM & VFFASWR \\
\hline P05164 & PERM & VVLEGGIDPILR \\
\hline P09619 & PGFRB & LPGFHGLR \\
\hline Q96PD5 & PGRP2 & DGSPDVTTADIGANTPDATK \\
\hline Q96PD5 & PGRP2 & EFTEAFLGCPAIHPR \\
\hline Q96PD5 & PGRP2 & TDCPGDALFDLLR \\
\hline Q96PD5 & PGRP2 & TFTLLDPK \\
\hline P80108 & PHLD & FGSSLITVR \\
\hline P80108 & PHLD & HVSSPLASYFLSFPYAR \\
\hline P80108 & PHLD & IADVTSGLIGGEDGR \\
\hline P80108 & PHLD & NQVVIAAGR \\
\hline P80108 & PHLD & SWITPCPEEK \\
\hline P80108 & PHLD & TLLLVGSPTWK \\
\hline Q6UXB8 & PI16 & WDEELAAFAK \\
\hline P02776 & PLF4 & ICLDLQAPLYK \\
\hline P00747 & PLMN & EAQLPVIENK \\
\hline P00747 & PLMN & LSSPAVITDK \\
\hline P00747 & PLMN & NPDGDVGGPWCYTTNPR \\
\hline P00747 & PLMN & VIPACLPSPNYVVADR \\
\hline P00747 & PLMN & WEYCNLK \\
\hline P00747 & PLMN & YEFLNGR \\
\hline P55058 & PLTP & ATYFGSIVLLSPAVIDSPLK \\
\hline P55058 & PLTP & AVEPQLQEEER \\
\hline P55058 & PLTP & FLEQELETITIPDLR \\
\hline
\end{tabular}

\begin{tabular}{|c|c|c|c|}
\hline 456.7 & 461.7 & 2 & 666.3 \\
\hline 640.9 & 645.9 & 2 & 840.5 \\
\hline 448.8 & 453.8 & 2 & 392.2 \\
\hline 973.5 & 977.5 & 2 & 874.4 \\
\hline 872.9 & 877.9 & 2 & 907.5 \\
\hline 746.9 & 751.9 & 2 & 1116.6 \\
\hline 467.8 & 471.8 & 2 & 244.2 \\
\hline 490.3 & 495.3 & 2 & 205.1 \\
\hline 648 & 651.3 & 3 & 740.4 \\
\hline 730.4 & 735.4 & 2 & 590.3 \\
\hline 464.3 & 469.3 & 2 & 586.4 \\
\hline 623.8 & 627.8 & 2 & 274.1 \\
\hline 607.9 & 611.9 & 2 & 215.1 \\
\hline 590.3 & 594.3 & 2 & 878.5 \\
\hline 667.4 & 671.4 & 2 & 274.1 \\
\hline 570.8 & 574.8 & 2 & 699.4 \\
\hline 515.8 & 519.8 & 2 & 743.4 \\
\hline 953.4 & 958.4 & 2 & 499.2 \\
\hline 886 & 891 & 2 & 1117.6 \\
\hline 506.7 & 510.7 & 2 & 697.3 \\
\hline 449.7 & 454.7 & 2 & 606.3 \\
\hline 1046.1 & 1050.1 & 2 & 1026.6 \\
\hline 664.3 & 669.3 & 2 & 514.8 \\
\hline 606.3 & 609.7 & 3 & 500.3 \\
\hline
\end{tabular}

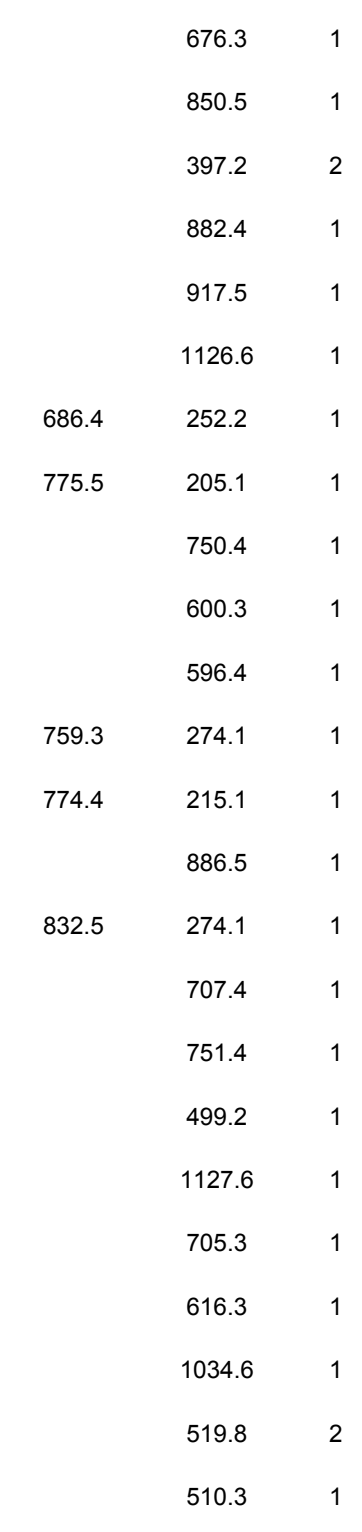

\begin{tabular}{|c|c|c|c|}
\hline y5 & & $Y$ & 15.2 \\
\hline y8 & & $Y$ & 20.9 \\
\hline y7 & & $Y$ & 14.9 \\
\hline y9 & & Y & 31.2 \\
\hline y8 & & $Y$ & 28.1 \\
\hline y10 & & $Y$ & 24.2 \\
\hline y2 & y6 & $Y$ & 15.5 \\
\hline b2 & y7 & $Y$ & 16.2 \\
\hline y6 & & $Y$ & 18.5 \\
\hline y6 & & $Y$ & 23.6 \\
\hline y6 & & $\mathrm{Y}$ & 15.4 \\
\hline b2 & y6 & $Y$ & 20.3 \\
\hline b2 & y7 & $Y$ & 19.8 \\
\hline y8 & & $Y$ & 19.3 \\
\hline b2 & y7 & Y & 21.7 \\
\hline y6 & & $Y$ & 18.7 \\
\hline y7 & & Y & 17 \\
\hline b5 & & $Y$ & 30.6 \\
\hline y10 & & $Y$ & 28.5 \\
\hline y5 & & $Y$ & 16.7 \\
\hline y5 & & $Y$ & 14.9 \\
\hline y10 & & $Y$ & 33.4 \\
\hline y8 & & $Y$ & 21.6 \\
\hline y4 & & $Y$ & 17 \\
\hline
\end{tabular}




\begin{tabular}{|l|l|l|} 
P55058 & PLTP & TGLELSR \\
\hline P27169 & PON1 & IFFYDSENPPASEVLR \\
\hline P27169 & PON1 & IHVYEK \\
\hline P27169 & PON1 & IQNILTEEPK \\
\hline P27169 & PON1 & SFNPNSPGK \\
\hline P27169 & PON1 & STVELFK \\
\hline P27169 & PON1 & VVAEGFDFANGINISPDGK \\
\hline P32119 & PRDX2 & TDEGIAYR \\
\hline P04070 & PROC & DTEDQEDQVDPR \\
\hline P04070 & PROC & GDSPWQVVLLDSK \\
\hline P04070 & PROC & TFVLNFIK \\
\hline P07737 & PROF1 & STGGAPTFNVTVTK \\
\hline P07225 & PROS & FSAEFDFR \\
\hline P07225 & PROS & HCLVTVEK \\
\hline P07225 & PROS & NNLELSTPLK \\
\hline P07225 & PROS & QSTNAYPDLR \\
\hline P07225 & PROS & SCEVVSVCLPLNLDTK \\
\hline P07225 & PROS & SFQTGLFTAAR \\
\hline P07225 & PROS & VYFAGFPR \\
\hline P20742 & PZP & ASPAFLASQNTK \\
\hline P20742 & PZP & GSFALSFPVESDVAPIAR \\
\hline P20742 & PZP & HQDGSYSTFGER \\
\hline P20742 & PZP & IQHPFTVEEFVLPK \\
\hline P20742 & PZP & LPSNVVK \\
\hline
\end{tabular}

\begin{tabular}{|c|c|c|c|}
\hline 388.2 & 393.2 & 2 & 504.3 \\
\hline 942.5 & 947.5 & 2 & 868.5 \\
\hline 394.7 & 398.7 & 2 & 338.2 \\
\hline 592.8 & 596.8 & 2 & 242.1 \\
\hline 474.2 & 478.2 & 2 & 599.3 \\
\hline 412.2 & 416.2 & 2 & 635.4 \\
\hline 975.5 & 979.5 & 2 & 503.2 \\
\hline 462.7 & 467.7 & 2 & 217.1 \\
\hline 723.8 & 728.8 & 2 & 272.2 \\
\hline 722.4 & 726.4 & 2 & 592.8 \\
\hline 491.3 & 495.3 & 2 & 733.5 \\
\hline 690.4 & 694.4 & 2 & 1006.6 \\
\hline 509.7 & 514.7 & 2 & 784.4 \\
\hline 493.3 & 497.3 & 2 & 848.5 \\
\hline 564.8 & 568.8 & 2 & 787.5 \\
\hline 582.8 & 587.8 & 2 & 500.3 \\
\hline 917.5 & 921.5 & 2 & 800.5 \\
\hline 599.8 & 604.8 & 2 & 836.5 \\
\hline 478.8 & 483.8 & 2 & 694.4 \\
\hline 617.8 & 621.8 & 2 & 648.3 \\
\hline 932 & 937 & 2 & 1153.6 \\
\hline 461.9 & 465.2 & 3 & 508.3 \\
\hline 562 & 564.6 & 3 & 244.2 \\
\hline 378.7 & 382.7 & 2 & 322.2 \\
\hline
\end{tabular}

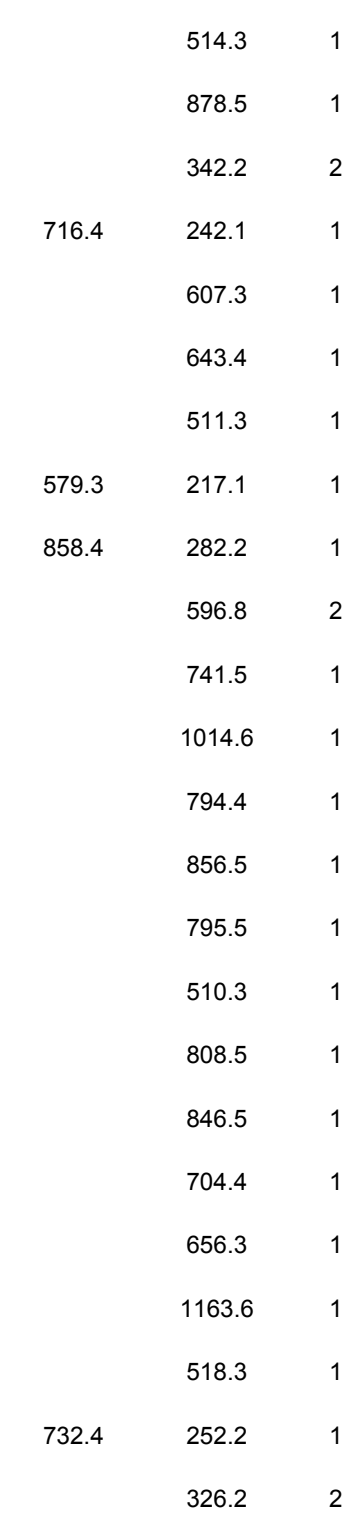




\begin{tabular}{|l|l|l|} 
P20742 & PZP & NALFCLESAWNVAK \\
\hline P00797 & RENI & LFDASDSSSYK \\
\hline P02753 & RET4 & LIVHNGYCDGR \\
\hline P02753 & RET4 & LLNLDGTCADSYSFVFSR \\
\hline P02753 & RET4 & QEELCLAR \\
\hline P02753 & RET4 & YWGVASFLQK \\
\hline P06702 & S10A9 & LGHPDTLNQGEFK \\
\hline P0DJI8 & SAA1 & FFGHGAEDSLADQAANEWG \\
\hline P02743 & SAMP & AYSLFSYNTQGR \\
\hline P02743 & SAMP & DNELLVYK \\
\hline P02743 & SAMP & IVLGQEQDSYGGK \\
\hline P02743 & SAMP & QGYFVEAQPK \\
\hline P02743 & SAMP & VFVFPR \\
\hline P02743 & SAMP & VGEYSLYIGR \\
\hline Q9BYB0 & SHAN3 & FEDHEIEGAHLPALTK \\
\hline P04278 & SHBG & DIPQPHAEPWAFSLDLGLK \\
\hline P04278 & SHBG & IALGGLLFPASNLR \\
\hline P04278 & SHBG & LPLVPALDGCLR \\
\hline P04278 & SHBG & TSSSFEVR \\
\hline P04278 & SHBG & VVLSQGSK \\
\hline Q13103 & SPP24 & DYYVSTAVCR \\
\hline P22105 & TENX & LQGLIPGAR \\
\hline P05452 & TETN & CFLAFTQTK \\
\hline P05452 & TETN & EQQALQTVCLK \\
\hline
\end{tabular}

$\begin{array}{cccc}811.9 & 815.9 & 2 & 299.2 \\ 610.3 & 614.3 & 2 & 959.4 \\ 652.3 & 657.3 & 2 & 489.7 \\ 689 & 692.3 & 3 & 742.4 \\ 509.8 & 514.8 & 2 & 519.3 \\ 599.8 & 603.8 & 2 & 849.5 \\ 485.9 & 488.6 & 3 & 621.3 \\ 726.7 & 730 & 3 & 732.3 \\ 703.8 & 708.8 & 2 & 825.4 \\ 497.3 & 501.3 & 2 & 522.3 \\ 697.4 & 701.4 & 2 & 1068.5 \\ 583.8 & 587.8 & 2 & 572.3 \\ 382.7 & 387.7 & 2 & 518.3 \\ 578.8 & 583.8 & 2 & 871.5 \\ 603 & 605.6 & 3 & 529.3 \\ 712 & 714.7 & 3 & 892.5 \\ 721.4 & 726.4 & 2 & 804.4 \\ 662.4 & 667.4 & 2 & 901.5 \\ 456.7 & 461.7 & 2 & 724.4 \\ 409.2 & 413.2 & 2 & 619.3 \\ 617.3 & 622.3 & 2 & 693.3 \\ 462.8 & 467.8 & 2 & 683.4 \\ 558.3 & 562.3 & 2 & 695.4 \\ 659.3 & 663.4 & 2 & 748.4\end{array}$

\begin{tabular}{ll}
299.2 & 1 \\
967.4 & 1 \\
494.7 & 2 \\
752.4 & 1 \\
529.3 & 1 \\
857.5 & 1 \\
621.3 & 1 \\
742.4 & 1 \\
835.4 & 1 \\
\hline 530.3 & 1 \\
1076.5 & 1 \\
5880.3 & 1 \\
\hline 528.3 & 1 \\
\hline 881.5 & 1 \\
\hline 537.3 & 1 \\
\hline 900.5 & 1 \\
\hline 814.4 & 1 \\
\hline 911.5 & 1 \\
734.4 & 1 \\
627.4 & 1 \\
703.3 & 1 \\
693.4 & 1 \\
703.4 & 1 \\
756.4 & 1
\end{tabular}

b3
$y 9$
$y 8$
$y 6$
$y 4$
$y 8$
$b 6$
$y 6$
$y 7$
$y 4$
$y 10$
$y 5$
$y 4$
$y 7$
$y 5$
$y 8$
$y 7$
$y 8$
$y 6$
$y 6$
$y 6$
$y 7$
$y 6$
$y 6$




\begin{tabular}{|l|l|l|} 
P05452 & TETN & LDTLAQEVALLK \\
\hline P05452 & TETN & TENCAVLSGAANGK \\
\hline P05452 & TETN & TFHEASEDCISR \\
\hline P05543 & THBG & AQWANPFDPSK \\
\hline P05543 & THBG & FLNDVK \\
\hline P05543 & THBG & GWVDLFVPK \\
\hline P05543 & THBG & NALALFVLPK \\
\hline P05543 & THBG & SILFLGK \\
\hline P00734 & THRB & ELLESYIDGR \\
\hline P00734 & THRB & HQDFNSAVQLVENFCR \\
\hline P00734 & THRB & LAVTTHGLPCLAWASAQAK \\
\hline P00734 & THRB & NPDSSTTGPWCYTTDPTVR \\
\hline P00734 & THRB & VTGWGNLK \\
\hline P00734 & THRB & YTACETAR \\
\hline P25942 & TNR5 & YCDPNLGLR \\
\hline P02787 & TRFE & ASYLDCIR \\
\hline P02787 & TRFE & CSTSSLLEACTFR \\
\hline P02787 & TRFE & FDEFFSEGCAPGSK \\
\hline P02787 & TRFE & HQTVPQNTGGK \\
\hline P02787 & TRFE & IECVSAETTEDCIAK \\
\hline P02787 & TRFE & SAGWNIPIGLLYCDLPEPR \\
\hline P02787 & TRFE & SASDLTWDNLK \\
\hline P02788 & TRFL & CSTSPLLEACEFLR \\
\hline P02766 & TTHY & AADDTWEPFASGK \\
\hline
\end{tabular}

\begin{tabular}{|c|c|c|c|}
\hline 657.4 & 661.4 & 2 & 871.5 \\
\hline 696.3 & 700.3 & 2 & 717.4 \\
\hline 484.5 & 487.9 & 3 & 375.2 \\
\hline 630.8 & 634.8 & 2 & 200.1 \\
\hline 368.2 & 372.2 & 2 & 475.3 \\
\hline 530.8 & 534.8 & 2 & 244.1 \\
\hline 543.3 & 547.3 & 2 & 787.5 \\
\hline 389.2 & 393.3 & 2 & 577.4 \\
\hline 597.8 & 602.8 & 2 & 710.3 \\
\hline 655.3 & 658.6 & 3 & 824.4 \\
\hline 665.7 & 668.4 & 3 & 832.4 \\
\hline 1078 & 1083 & 2 & 472.3 \\
\hline 437.7 & 441.7 & 2 & 674.4 \\
\hline 486.2 & 491.2 & 2 & 707.3 \\
\hline 554.3 & 559.3 & 2 & 669.4 \\
\hline 499.2 & 504.2 & 2 & 563.3 \\
\hline 766.3 & 771.4 & 2 & 783.3 \\
\hline 526.6 & 529.2 & 3 & 676.3 \\
\hline 389.5 & 392.2 & 3 & 351.2 \\
\hline 863.4 & 867.4 & 2 & 742.3 \\
\hline 724.4 & 727.7 & 3 & 498.3 \\
\hline 417.2 & 419.9 & 3 & 675.3 \\
\hline 561.6 & 564.9 & 3 & 795.4 \\
\hline 697.8 & 701.8 & 2 & 606.3 \\
\hline
\end{tabular}

$\begin{array}{ccc} & 879.5 & 1 \\ & 725.4 & 1 \\ & 385.2 & 1 \\ 718.4 & 200.1 & 1 \\ & 483.3 & 1 \\ & 244.1 & 1 \\ 795.5 & 1 \\ 585.4 & 1 \\ 720.4 & 1 \\ 834.4 & 1 \\ 840.4 & 1 \\ 482.3 & 1 \\ 682.4 & 1 \\ 717.3 & 1 \\ 679.4 & 1 \\ 573.3 & 1 \\ 793.4 & 1 \\ 684.3 & 1 \\ 355.2 & 2 \\ 746.3 & 2 \\ 508.3 & 1 \\ 688.4 & 1 \\ 805.4 & 1 \\ 614.3 & 1\end{array}$

\begin{tabular}{|c|c|c|c|}
\hline y8 & & $Y$ & 21.4 \\
\hline y8 & & $Y$ & 22.6 \\
\hline y3 & & $Y$ & 12.6 \\
\hline b2 & $y 4$ & $Y$ & 20.6 \\
\hline y4 & & $Y$ & 12.4 \\
\hline b2 & y6 & $Y$ & 17.5 \\
\hline y7 & & $Y$ & 17.8 \\
\hline y5 & & $Y$ & 13.1 \\
\hline y6 & & $Y$ & 19.5 \\
\hline y6 & & $Y$ & 18.8 \\
\hline y8 & & $Y$ & 19.2 \\
\hline y4 & & $Y$ & 34.4 \\
\hline y6 & & $Y$ & 14.6 \\
\hline y6 & & $Y$ & 16.1 \\
\hline y6 & & $Y$ & 18.2 \\
\hline y4 & & $Y$ & 16.5 \\
\hline y6 & & $Y$ & 24.8 \\
\hline y7 & & $Y$ & 14.2 \\
\hline y7 & & $Y$ & 9.2 \\
\hline y13 & & $Y$ & 27.8 \\
\hline y4 & & $Y$ & 21.3 \\
\hline y5 & & $Y$ & 10.2 \\
\hline y6 & & $Y$ & 15.4 \\
\hline y6 & & $Y$ & 22.6 \\
\hline
\end{tabular}




\begin{tabular}{|l|l|l|} 
P02766 & TTHY & GSPAINVAVHVFR \\
\hline P02766 & TTHY & VLDAVR \\
\hline P07911 & UROM & SGSVIDQSR \\
\hline P07911 & UROM & VLNLGPITR \\
\hline Q86UX7 & URP2 & VVLAGGVAPALFR \\
\hline Q6EMK4 & VASN & LAGLGLQQLDEGLFSR \\
\hline Q6EMK4 & VASN & SLTLGIEPVSPTSLR \\
\hline Q6EMK4 & VASN & YLQGSSVQLR \\
\hline P35916 & VGFR3 & SGVDLADSNQK \\
\hline P18206 & VINC & QVATALQNLQTK \\
\hline P02774 & VTDB & FEDCCQEK \\
\hline P02774 & VTDB & HLSLLTTLSNR \\
\hline P02774 & VTDB & THLPEVFLSK \\
\hline P02774 & VTDB & VCSQYAAYGEK \\
\hline P02774 & VTDB & YTFELSR \\
\hline P04004 & VTNC & CTEGFNVDK \\
\hline P04004 & VTNC & DVWGIEGPIDAAFTR \\
\hline P04004 & VTNC & FEDGVLDPDYPR \\
\hline P04004 & VTNC & GSQYWR \\
\hline P04004 & VTNC & VDTVDPPYPR \\
\hline P04275 & VWF & CHPLVDPEPFVALCEK \\
\hline P04275 & VWF & VTVFPIGIGDR \\
\hline P25311 & ZA2G & AGEVQEPELR \\
\hline P25311 & ZA2G & AYLEEECPATLR \\
\hline
\end{tabular}

\begin{tabular}{|c|c|c|c|}
\hline 456.3 & 459.6 & 3 & 558.3 \\
\hline 336.7 & 341.7 & 2 & 460.3 \\
\hline 474.7 & 479.7 & 2 & 618.3 \\
\hline 491.8 & 496.8 & 2 & 770.5 \\
\hline 635.4 & 640.4 & 2 & 887.5 \\
\hline 573 & 576.3 & 3 & 823.4 \\
\hline 785.4 & 790.5 & 2 & 856.5 \\
\hline 575.8 & 580.8 & 2 & 746.4 \\
\hline 567.3 & 571.3 & 2 & 662.3 \\
\hline 657.9 & 661.9 & 2 & 844.5 \\
\hline 558.2 & 562.2 & 2 & 839.3 \\
\hline 418.9 & 422.2 & 3 & 590.3 \\
\hline 585.8 & 589.8 & 2 & 239.1 \\
\hline 638.3 & 642.3 & 2 & 1016.5 \\
\hline 458.2 & 463.2 & 2 & 651.3 \\
\hline 535.2 & 539.2 & 2 & 808.4 \\
\hline 823.9 & 828.9 & 2 & 458.2 \\
\hline 711.8 & 716.8 & 2 & 647.3 \\
\hline 398.7 & 403.7 & 2 & 524.3 \\
\hline 579.8 & 584.8 & 2 & 629.3 \\
\hline 637.6 & 640.3 & 3 & 722.3 \\
\hline 587.3 & 592.3 & 2 & 727.4 \\
\hline 564.3 & 569.3 & 2 & 771.4 \\
\hline 726.3 & 731.3 & 2 & 235.1 \\
\hline
\end{tabular}

\begin{tabular}{|c|c|c|}
\hline \multicolumn{2}{|r|}{568.3} & 1 \\
\hline & 470.3 & 1 \\
\hline & 628.3 & 1 \\
\hline & 780.5 & 1 \\
\hline & 897.5 & 1 \\
\hline & 833.4 & 1 \\
\hline & 866.5 & 1 \\
\hline & 756.4 & 1 \\
\hline & 670.3 & 1 \\
\hline & 852.5 & 1 \\
\hline & 847.3 & 1 \\
\hline & 600.3 & 1 \\
\hline 819.5 & 239.1 & 1 \\
\hline & 1024.5 & 1 \\
\hline & 661.4 & 1 \\
\hline & 816.4 & 1 \\
\hline & 458.2 & 1 \\
\hline & 657.3 & 1 \\
\hline & 534.3 & 1 \\
\hline & 639.3 & 1 \\
\hline & 722.3 & 1 \\
\hline & 737.4 & 1 \\
\hline & 781.4 & 1 \\
\hline 846.4 & 235.1 & . \\
\hline
\end{tabular}




\begin{tabular}{|c|c|c|c|c|c|c|c|c|c|c|c|}
\hline P25311 & ZA2G & CLAYDFYPGK & 617.3 & 621.3 & 2 & 301.2 & 309.2 & 1 & y3 & Y & 20.1 \\
\hline P25311 & ZA2G & QDPPSVVVTSHQAPGEK & 592.6 & 595.3 & 3 & 766.9 & 770.9 & 2 & y15 & $\mathrm{Y}$ & 16.5 \\
\hline P25311 & ZA2G & SSGAFWK & 391.7 & 395.7 & 2 & 608.3 & 616.3 & 1 & y5 & $\mathrm{Y}$ & 13.1 \\
\hline P25311 & ZA2G & YSLTYIYTGLSK & 704.9 & 708.9 & 2 & 781.4 & 789.5 & 1 & y7 & $\mathrm{Y}$ & 22.9 \\
\hline
\end{tabular}

${ }^{a}$ UniProt accession number, protein ID, and information pertaining to mass spectra are listed for each peptide.

${ }^{\mathrm{b}}$ For quantifiers (product ions) that are less than $300 \mathrm{~m} / \mathrm{z}$, additional quantifiers $(>300 \mathrm{~m} / \mathrm{z}$ ) and their corresponding ion charge and type are listed.

${ }^{c}$ All product ions are specified as quantifiers. Y, yes. 
Supplementary Table S2. The 210 proteins (210 peptides) selected as candidate features in the training set ${ }^{\mathrm{a}}$

\begin{tabular}{|c|c|c|c|}
\hline $\begin{array}{c}\text { Uniprot accession } \\
\text { number }\end{array}$ & Protein & Peptide & $\begin{array}{l}\text { AUROC value } \\
\text { (MDD vs BD) }\end{array}$ \\
\hline P02763 & $\mathrm{A} 1 \mathrm{AG} 1$ & SDVVYTDWK & 0.426 \\
\hline P19652 & A1AG2 & EQLGEFYEALDCLCIPR & 0.467 \\
\hline P04217 & A1BG & CEGPIPDVTFELLR & 0.564 \\
\hline P08697 & A2AP & QEDDLANINQWVK & 0.557 \\
\hline P01023 & A2MG & VYDYYETDEFAIAEYNAPCSK & 0.55 \\
\hline Q15848 & ADIPO & GDIGETGVPGAEGPR & 0.521 \\
\hline P43652 & AFAM & HFQNLGK & 0.402 \\
\hline P02768 & ALBU & LVNEVTEFAK & 0.509 \\
\hline P04075 & ALDOA & ALQASALK & 0.386 \\
\hline P35858 & ALS & LHSLHLEGSCLGR & 0.461 \\
\hline P02760 & AMBP & CVLFPYGGCQGNGNK & 0.6 \\
\hline P15144 & AMPN & AQIINDAFNLASAHK & 0.39 \\
\hline P54802 & ANAG & DFCGCHVAWSGSQLR & 0.49 \\
\hline P01019 & ANGT & LQAILGVPWK & 0.425 \\
\hline P01008 & ANT3 & VWELSK & 0.442 \\
\hline P08519 & APOA & NPDAVAAPYCYTR & 0.577 \\
\hline P02647 & APOA1 & QGLLPVLESFK & 0.416 \\
\hline P02652 & APOA2 & SPELQAEAK & 0.406 \\
\hline P06727 & APOA4 & GNTEGLQK & 0.564 \\
\hline P04114 & APOB & ITLPDFR & 0.441 \\
\hline P02655 & APOC2 & ESLSSYWESAK & 0.444 \\
\hline P02656 & APOC3 & DYWSTVK & 0.405 \\
\hline P05090 & APOD & VLNQELR & 0.399 \\
\hline P02649 & APOE & EQVAEVR & 0.453 \\
\hline Q13790 & APOF & SLPTEDCENEK & 0.473 \\
\hline P02749 & $\mathrm{APOH}$ & ATVVYQGER & 0.441 \\
\hline 014791 & APOL1 & LNILNNNYK & 0.555 \\
\hline 095445 & APOM & FLLYNR & 0.39 \\
\hline P00966 & ASSY & IDIVENR & 0.487 \\
\hline Q76LX8 & ATS13 & LFINVAPHAR & 0.542 \\
\hline P61769 & B2MG & VNHVTLSQPK & 0.573 \\
\hline P02730 & B3AT & LSVPDGFK & 0.432 \\
\hline
\end{tabular}




\begin{tabular}{|c|c|c|c|}
\hline Q8TDL5 & BPIB1 & ALGFEAAESSLTK & 0.496 \\
\hline P43251 & BTD & VDLITFDTPFAGR & 0.476 \\
\hline Q06187 & BTK & LVQLYGVCTK & 0.417 \\
\hline P02745 & C1QA & SLGFCDTTNK & 0.568 \\
\hline P02746 & $\mathrm{C} 1 \mathrm{QB}$ & LEQGENVFLQATDK & 0.629 \\
\hline P02747 & C1QC & QTHQPPAPNSLIR & 0.619 \\
\hline P00736 & $\mathrm{C} 1 \mathrm{R}$ & NIGEFCGK & 0.527 \\
\hline Q9NZP8 & C1RL & GSEAINAPGDNPAK & 0.564 \\
\hline P09871 & C1S & CEYQIR & 0.604 \\
\hline P04003 & C4BPA & LSCSYSHWSAPAPQCK & 0.596 \\
\hline P54289 & CA2D1 & VLLDAGFTNELVQNYWSK & 0.514 \\
\hline P00915 & $\mathrm{CAH} 1$ & GGPFSDSYR & 0.486 \\
\hline P00918 & $\mathrm{CAH} 2$ & YGDFGK & 0.454 \\
\hline P27797 & CALR & FVLSSGK & 0.411 \\
\hline P08185 & CBG & HLVALSPK & 0.498 \\
\hline P22681 & $\mathrm{CBL}$ & GTEPIVVDPFDPR & 0.461 \\
\hline Q96IY4 & CBPB2 & YPLYVLK & 0.433 \\
\hline P15169 & CBPN & VQNECPGITR & 0.538 \\
\hline P30279 & CCND2 & ACQEQIEAVLLNSLQQYR & 0.445 \\
\hline P08571 & CD14 & VLDLSCNR & 0.574 \\
\hline O43866 & CD5L & CYGPGVGR & 0.619 \\
\hline P06731 & CEAM5 & TLTLFNVTR & 0.481 \\
\hline P00450 & CERU & EYTDASFTNR & 0.393 \\
\hline P00751 & CFAB & VSEADSSNADWVTK & 0.454 \\
\hline P08603 & CFAH & CVEISCK & 0.576 \\
\hline P05156 & CFAI & EANVACLDLGFQQGADTQR & 0.53 \\
\hline P06276 & CHLE & AEEILSR & 0.525 \\
\hline P10909 & CLUS & EIQNAVNGVK & 0.521 \\
\hline Q96KN2 & CNDP1 & AlHLDLEEYR & 0.46 \\
\hline P02452 & Co1A1 & VLCDDVICDETK & 0.518 \\
\hline P06681 & $\mathrm{CO} 2$ & HAFILQDTK & 0.458 \\
\hline P01024 & $\mathrm{CO} 3$ & DSCVGSLVVK & 0.556 \\
\hline POCOL4 & CO4A & ANSFLGEK & 0.468 \\
\hline P08572 & CO4A2 & IAVQPGTVGPQGR & 0.55 \\
\hline P01031 & $\operatorname{co5}$ & IDTALIK & 0.427 \\
\hline P13671 & $\mathrm{CO} 6$ & GFVVAGPSR & 0.57 \\
\hline P10643 & $\mathrm{CO} 7$ & VLFYVDSEK & 0.545 \\
\hline
\end{tabular}




\begin{tabular}{|c|c|c|c|}
\hline P07357 & $\mathrm{CO} A \mathrm{~A}$ & AIDEDCSQYEPIPGSQK & 0.474 \\
\hline P07358 & $\mathrm{CO} B \mathrm{~B}$ & CEGFVCAQTGR & 0.572 \\
\hline P07360 & CO8G & AGQLSVK & 0.503 \\
\hline P02748 & $\operatorname{cog}$ & TSNFNAAISLK & 0.586 \\
\hline Q03692 & COAA1 & GTHVWVGLYK & 0.41 \\
\hline Q9UMD9 & COHA1 & QAAYNADSGLK & 0.482 \\
\hline P49747 & COMP & DTDLDGFPDEK & 0.524 \\
\hline P20815 & CP3A5 & DTINFLSK & 0.516 \\
\hline P22792 & CPN2 & QLVCPVTR & 0.536 \\
\hline P02741 & CRP & ESDTSYVSLK & 0.491 \\
\hline P02775 & CXCL7 & NIQSLEVIGK & 0.359 \\
\hline P01034 & CYTC & ALDFAVGEYNK & 0.474 \\
\hline O95822 & DCMC & LCAWYLYGEK & 0.455 \\
\hline P09172 & DOPO & VISTLEEPTPQCPTSQGR & 0.577 \\
\hline Q14126 & DSG2 & ILDVNDNIPVVENK & 0.448 \\
\hline P32926 & DSG3 & LAEISLGVDGEGK & 0.328 \\
\hline Q16610 & ECM1 & FCEAEFSVK & 0.605 \\
\hline P00533 & EGFR & CNLLEGEPR & 0.52 \\
\hline Q01780 & EXOSX & SGPLPSAER & 0.572 \\
\hline P00488 & F13A & STVLTIPEIIIK & 0.427 \\
\hline P05160 & F13B & VLHGDLIDFVCK & 0.537 \\
\hline P00742 & FA10 & QEDACQGDSGGPHVTR & 0.593 \\
\hline P03951 & FA11 & SCALSNLACIR & 0.572 \\
\hline P00748 & FA12 & CFEPQLLR & 0.547 \\
\hline P12259 & FA5 & GEYEEHLGILGPIIR & 0.574 \\
\hline P08709 & FA7 & LHQPVVLTDHVVPLCLPER & 0.515 \\
\hline P00740 & FA9 & NCELDVTCNIK & 0.563 \\
\hline P23142 & FBLN1 & CVDVDECAPPAEPCGK & 0.646 \\
\hline Q12805 & FBLN3 & NPCQDPYILTPENR & 0.553 \\
\hline P35556 & FBN2 & FNLSHLGSK & 0.516 \\
\hline P22087 & FBRL & NGGHFVISIK & 0.575 \\
\hline P08637 & FCG3A & AVVFLEPQWYR & 0.478 \\
\hline Q9Y6R7 & FCGBP & AIGYATAADCGR & 0.602 \\
\hline O75636 & FCN3 & YGIDWASGR & 0.474 \\
\hline P02765 & FETUA & CNLLAEK & 0.548 \\
\hline Q9UGM5 & FETUB & SQASSCSLQSSDSVPVGLCK & 0.443 \\
\hline Q03591 & FHR1 & TGESAEFVCK & 0.482 \\
\hline
\end{tabular}




\begin{tabular}{|c|c|c|c|}
\hline Q02985 & FHR3 & AQTTVTCTEK & 0.595 \\
\hline Q9BXR6 & FHR5 & TGDAVEFQCK & 0.528 \\
\hline P02671 & FIBA & VQHIQLLQK & 0.46 \\
\hline P02675 & FIBB & QGFGNVATNTDGK & 0.527 \\
\hline P02679 & FIBG & ASTPNGYDNGIIWATWK & 0.465 \\
\hline P02751 & FINC & VPGTSTSATLTGLTR & 0.458 \\
\hline Q06787 & FMR1 & EPCCWWLAK & 0.439 \\
\hline O95954 & FTCD & SDLQVAAK & 0.498 \\
\hline P06396 & GELS & QTQVSVLPEGGETPLFK & 0.549 \\
\hline Q92820 & GGH & YLESAGAR & 0.487 \\
\hline P22352 & GPX3 & NSCPPTSELLGTSDR & 0.352 \\
\hline Q14520 & HABP2 & FCEIGSDDCYVGDGYSYR & 0.612 \\
\hline P69905 & HBA & VGAHAGEYGAEALER & 0.509 \\
\hline P08397 & HEM3 & ELEHALEK & 0.395 \\
\hline P02790 & HEMO & SGAQATWTELPWPHEK & 0.441 \\
\hline P05546 & HEP2 & TLEAQLTPR & 0.419 \\
\hline Q04756 & HGFA & LEACESLTR & 0.602 \\
\hline P00738 & HPT & VGYVSGWGR & 0.492 \\
\hline P00739 & HPTR & VVLHPNYHQVDIGLIK & 0.518 \\
\hline P04196 & HRG & QIGSVYR & 0.546 \\
\hline P18065 & IBP2 & LIQGAPTIR & 0.415 \\
\hline P17936 & IBP3 & YGQPLPGYTTK & 0.543 \\
\hline P24593 & IBP5 & GVCLNEK & 0.529 \\
\hline P05155 & IC1 & LLDSLPSDTR & 0.556 \\
\hline P05362 & ICAM1 & VELAPLPSWQPVGK & 0.56 \\
\hline P22304 & IDS & QSTEQAIQLLEK & 0.467 \\
\hline P01344 & IGF2 & GIVEECCFR & 0.586 \\
\hline P01857 & IGHG1 & TPEVTCVVVDVSHEDPEVK & 0.529 \\
\hline P01860 & IGHG3 & SCDTPPPCPR & 0.538 \\
\hline P01871 & IGHM & QVGSGVTTDQVQAEAK & 0.598 \\
\hline P01834 & IGKC & DSTYSLSSTLTLSK & 0.563 \\
\hline P05113 & IL5 & ETLALLSTHR & 0.443 \\
\hline P06213 & INSR & VCHLLEGEK & 0.539 \\
\hline P05154 & IPSP & AAAATGTIFTFR & 0.596 \\
\hline P08514 & ITA2B & IVLLDVPVR & 0.383 \\
\hline P19827 & $\mathrm{ITIH} 1$ & GSLVQASEANLQAAQDFVR & 0.404 \\
\hline P19823 & ITIH2 & IYLQPGR & 0.403 \\
\hline
\end{tabular}




\begin{tabular}{|c|c|c|c|}
\hline Q06033 & $\mathrm{ITIH} 3$ & EVSFDVELPK & 0.536 \\
\hline Q14624 & ITIH4 & NVVFVIDK & 0.386 \\
\hline 060674 & JAK2 & SDNIIFQFTK & 0.535 \\
\hline P35527 & $\mathrm{K} 1 \mathrm{C} 9$ & TLLDIDNTR & 0.453 \\
\hline P04264 & $\mathrm{K} 2 \mathrm{C} 1$ & SLVNLGGSK & 0.404 \\
\hline P13647 & $\mathrm{K} 2 \mathrm{C} 5$ & ISISTSGGSFR & 0.469 \\
\hline P29622 & KAIN & GFQHLLHTLNLPGHGLETR & 0.58 \\
\hline P06732 & KCRM & ELFDPIISDR & 0.455 \\
\hline O75037 & $\mathrm{KI} 21 \mathrm{~B}$ & AQEQGVAGPEFK & 0.442 \\
\hline P03952 & KLKB1 & LVGITSWGEGCAR & 0.442 \\
\hline P01042 & KNG1 & QVVAGLNFR & 0.55 \\
\hline P05455 & LA & IGCLLK & 0.402 \\
\hline P11279 & LAMP1 & ALQATVGNSYK & 0.435 \\
\hline P13473 & LAMP2 & IPLNDLFR & 0.526 \\
\hline P18428 & LBP & GLQYAAQEGLLALQSELLR & 0.554 \\
\hline P04180 & LCAT & SSGLVSNAPGVQIR & 0.607 \\
\hline P07195 & LDHB & SADTLWDIQK & 0.366 \\
\hline P51884 & LUM & LPSGLPVSLLTLYLDNNK & 0.464 \\
\hline P14151 & LYAM1 & AEIEYLEK & 0.53 \\
\hline Q86UE4 & LYRIC & WNSVSPASAGK & 0.488 \\
\hline P61626 & LYSC & STDYGIFQINSR & 0.479 \\
\hline P11226 & MBL2 & WLTFSLGK & 0.545 \\
\hline O43772 & MCAT & EGITGLYR & 0.511 \\
\hline Q16674 & MIA & GQVVYVFSK & 0.464 \\
\hline P43246 & $\mathrm{MSH} 2$ & DIYQDLNR & 0.478 \\
\hline P61916 & NPC2 & SEYPSIK & 0.447 \\
\hline Q13093 & PAFA & ASLAFLQK & 0.426 \\
\hline P36955 & PEDF & ALYYDLISSPDIHGTYK & 0.538 \\
\hline P05164 & PERM & VVLEGGIDPILR & 0.421 \\
\hline P09619 & PGFRB & LPGFHGLR & 0.557 \\
\hline Q96PD5 & PGRP2 & EFTEAFLGCPAIHPR & 0.527 \\
\hline P80108 & PHLD & TLLLVGSPTWK & 0.468 \\
\hline Q6UXB8 & PI16 & WDEELAAFAK & 0.562 \\
\hline P02776 & PLF4 & ICLDLQAPLYK & 0.351 \\
\hline P00747 & PLMN & YEFLNGR & 0.435 \\
\hline P55058 & PLTP & AVEPQLQEEER & 0.431 \\
\hline P27169 & PON1 & IHVYEK & 0.518 \\
\hline
\end{tabular}




\begin{tabular}{|c|c|c|c|}
\hline P32119 & PRDX2 & TDEGIAYR & 0.475 \\
\hline P04070 & PROC & GDSPWQVVLLDSK & 0.39 \\
\hline P07737 & PROF1 & STGGAPTFNVTVTK & 0.403 \\
\hline P07225 & PROS & VYFAGFPR & 0.459 \\
\hline P20742 & PZP & HQDGSYSTFGER & 0.507 \\
\hline P00797 & RENI & LFDASDSSSYK & 0.515 \\
\hline P02753 & RET4 & QEELCLAR & 0.542 \\
\hline P06702 & S10A9 & LGHPDTLNQGEFK & 0.434 \\
\hline P0DJI8 & SAA1 & FFGHGAEDSLADQAANEWGR & 0.558 \\
\hline P02743 & SAMP & IVLGQEQDSYGGK & 0.445 \\
\hline Q9BYB0 & SHAN3 & FEDHEIEGAHLPALTK & 0.517 \\
\hline P04278 & SHBG & TSSSFEVR & 0.468 \\
\hline Q13103 & SPP24 & DYYVSTAVCR & 0.373 \\
\hline P22105 & TENX & LQGLIPGAR & 0.558 \\
\hline P05452 & TETN & CFLAFTQTK & 0.612 \\
\hline P05543 & THBG & NALALFVLPK & 0.53 \\
\hline P00734 & THRB & VTGWGNLK & 0.443 \\
\hline P25942 & TNR5 & YCDPNLGLR & 0.469 \\
\hline P02787 & TRFE & CSTSSLLEACTFR & 0.62 \\
\hline P02788 & TRFL & CSTSPLLEACEFLR & 0.498 \\
\hline P02766 & TTHY & AADDTWEPFASGK & 0.391 \\
\hline P07911 & UROM & VLNLGPITR & 0.394 \\
\hline Q86UX7 & URP2 & VVLAGGVAPALFR & 0.36 \\
\hline Q6EMK4 & VASN & LAGLGLQQLDEGLFSR & 0.556 \\
\hline P35916 & VGFR3 & SGVDLADSNQK & 0.476 \\
\hline P18206 & VINC & QVATALQNLQTK & 0.364 \\
\hline P02774 & VTDB & YTFELSR & 0.448 \\
\hline P04004 & VTNC & CTEGFNVDK & 0.616 \\
\hline P04275 & VWF & VTVFPIGIGDR & 0.447 \\
\hline P25311 & ZA2G & CLAYDFYPGK & 0.561 \\
\hline
\end{tabular}

a Uniprot accession number, protein ID, and AUROC value for each corresponding peptide. Abbreviations: AUROC, area under the receiver operating characteristics; MDD, major depressive disorder; BD, bipolar disorder 
${ }^{\mathrm{b}}$ Because MDD and BD were labeled 0 and 1, proteins and their corresponding peptides in the range of AUROC values (0.5-1) indicate that they can more elaborately classify BD as BD itself compared with MDD (light yellow). Proteins and their corresponding peptides in the range of AUROC values (0-0.5) signify that they can more precisely classify MDD as MDD itself compared with BD (light blue). The AUROC values (0-0.5) are the same as those (0.5-1) when BD and MDD are designated 0 and 1, respectively. 
Supplementary Table S3. Proportion of features selected for the 210 candidate features ( 210 proteins $/ 210$ peptides) used to determine the model for discriminating MDD from $\mathrm{BD}^{\mathrm{a}}$

\begin{tabular}{|c|c|}
\hline Feature & Proportion of feature selected \\
\hline \multicolumn{2}{|l|}{ Protein_Peptide sequence } \\
\hline A1AG1_SDVVYTDWK & 0 \\
\hline A1AG2_EQLGEFYEALDCLCIPR & 0 \\
\hline A1BG_CEGPIPDVTFELLR & 0 \\
\hline A2AP_QEDDLANINQWVK & 0 \\
\hline A2MG_VYDYYETDEFAIAEYNAPCSK & 0 \\
\hline ADIPO_GDIGETGVPGAEGPR & 0 \\
\hline AFAM_HFQNLGK & 0 \\
\hline ALBU_LVNEVTEFAK & 0 \\
\hline ALDOA_ALQASALK & 0.87 \\
\hline ALS_LHSLHLEGSCLGR & 0 \\
\hline AMBP_CVLFPYGGCQGNGNK & 0 \\
\hline AMPN_AQIINDAFNLASAHK & 0.12 \\
\hline ANAG_DFCGCHVAWSGSQLR & 0 \\
\hline ANGT_LQAILGVPWK & 0 \\
\hline ANT3_VWELSK & 0 \\
\hline APOA_NPDAVAAPYCYTR & 0 \\
\hline APOA1_QGLLPVLESFK & 0 \\
\hline APOA2_SPELQAEAK & 0 \\
\hline APOA4_GNTEGLQK & 0 \\
\hline APOB_ITLPDFR & 0 \\
\hline APOC2_ESLSSYWESAK & 0 \\
\hline APOC3_DYWSTVK & 0 \\
\hline APOD_VLNQELR & 0 \\
\hline APOE_EQVAEVR & 0 \\
\hline APOF_SLPTEDCENEK & 0 \\
\hline APOH_ATVVYQGER & 0 \\
\hline APOL1_LNILNNNYK & 0 \\
\hline APOM_FLLYNR & 0 \\
\hline ASSY_IDIVENR & 0 \\
\hline ATS13_LFINVAPHAR & 0 \\
\hline B2MG_VNHVTLSQPK & 0 \\
\hline B3AT_LSVPDGFK & 0 \\
\hline BPIB1_ALGFEAAESSLTK & 0 \\
\hline
\end{tabular}




\begin{tabular}{|c|c|}
\hline BTD_VDLITFDTPFAGR & 0 \\
\hline BTK_LVQLYGVCTK & 0 \\
\hline C1QA_SLGFCDTTNK & 0 \\
\hline C1QB_LEQGENVFLQATDK & 1 \\
\hline C1QC_QTHQPPAPNSLIR & 0 \\
\hline C1R_NIGEFCGK & 0 \\
\hline C1RL_GSEAINAPGDNPAK & 0 \\
\hline C1S_CEYQIR & 0 \\
\hline C4BPA_LSCSYSHWSAPAPQCK & 0 \\
\hline CA2D1_VLLDAGFTNELVQNYWSK & 0 \\
\hline CAH1_GGPFSDSYR & 0 \\
\hline CAH2_YGDFGK & 0 \\
\hline CALR_FVLSSGK & 0 \\
\hline CBG_HLVALSPK & 0 \\
\hline CBL_GTEPIVVDPFDPR & 0 \\
\hline CBPB2_YPLYVLK & 0 \\
\hline CBPN_VQNECPGITR & 0 \\
\hline CCND2_ACQEQIEAVLLNSLQQYR & 0 \\
\hline CD14_VLDLSCNR & 0 \\
\hline CD5L_CYGPGVGR & 0 \\
\hline CEAM5_TLTLFNVTR & 0 \\
\hline CERU_EYTDASFTNR & 0.03 \\
\hline CFAB_VSEADSSNADWVTK & 0 \\
\hline CFAH_CVEISCK & 0 \\
\hline CFAI_EANVACLDLGFQQGADTQR & 0 \\
\hline CHLE_AEEILSR & 0 \\
\hline CLUS_EIQNAVNGVK & 0 \\
\hline CNDP1_AIHLDLEEYR & 0 \\
\hline CO1A1_VLCDDVICDETK & 0 \\
\hline CO2_HAFILQDTK & 0 \\
\hline CO3_DSCVGSLVVK & 0 \\
\hline CO4A_ANSFLGEK & 0 \\
\hline CO4A2_IAVQPGTVGPQGR & 0 \\
\hline CO5_IDTALIK & 0 \\
\hline CO6_GFVVAGPSR & 0 \\
\hline CO7_VLFYVDSEK & 0 \\
\hline CO8A_AIDEDCSQYEPIPGSQK & 0 \\
\hline CO8B_CEGFVCAQTGR & 0 \\
\hline CO8G_AGQLSVK & 0 \\
\hline CO9_TSNFNAAISLK & 0 \\
\hline COAA1_GTHVWVGLYK & 0.22 \\
\hline COHA1_QAAYNADSGLK & 0 \\
\hline COMP_DTDLDGFPDEK & 0 \\
\hline CP3A5_DTINFLSK & 0 \\
\hline
\end{tabular}




\begin{tabular}{|c|c|}
\hline CPN2_QLVCPVTR & 0 \\
\hline CRP_ESDTSYVSLK & 0 \\
\hline CXCL7_NIQSLEVIGK & 0.04 \\
\hline CYTC_ALDFAVGEYNK & 0 \\
\hline DCMC_LCAWYLYGEK & 0 \\
\hline DOPO_VISTLEEPTPQCPTSQGR & 0 \\
\hline DSG2_ILDVNDNIPVVENK & 0 \\
\hline DSG3_LAEISLGVDGEGK & 1 \\
\hline ECM1_FCEAEFSVK & 0 \\
\hline EGFR_CNLLEGEPR & 0 \\
\hline EXOSX_SGPLPSAER & 0 \\
\hline F13A_STVLTIPEIIIK & 0 \\
\hline F13B_VLHGDLIDFVCK & 0 \\
\hline FA10_QEDACQGDSGGPHVTR & 0.37 \\
\hline FA11_SCALSNLACIR & 0 \\
\hline FA12_CFEPQLLR & 0 \\
\hline FA5_GEYEEHLGILGPIIR & 0 \\
\hline FA7_LHQPVVLTDHVVPLCLPER & 0 \\
\hline FA9_NCELDVTCNIK & 0.87 \\
\hline FBLN1_CVDVDECAPPAEPCGK & 1 \\
\hline FBLN3_NPCQDPYILTPENR & 0 \\
\hline FBN2_FNLSHLGSK & 0 \\
\hline FBRL_NGGHFVISIK & 0 \\
\hline FCG3A_AVVFLEPQWYR & 0 \\
\hline FCGBP_AIGYATAADCGR & 1 \\
\hline FCN3_YGIDWASGR & 0 \\
\hline FETUA_CNLLAEK & 0 \\
\hline FETUB_SQASSCSLQSSDSVPVGLCK & 0 \\
\hline FHR1_TGESAEFVCK & 0 \\
\hline FHR3_AQTTVTCTEK & 1 \\
\hline FHR5_TGDAVEFQCK & 0 \\
\hline FIBA_VQHIQLLQK & 0 \\
\hline FIBB_QGFGNVATNTDGK & 0 \\
\hline FIBG_ASTPNGYDNGIIWATWK & 0 \\
\hline FINC_VPGTSTSATLTGLTR & 0 \\
\hline FMR1_EPCCWWLAK & 0 \\
\hline FTCD_SDLQVAAK & 0 \\
\hline GELS_QTQVSVLPEGGETPLFK & 0 \\
\hline GGH_YLESAGAR & 0 \\
\hline GPX3_NSCPPTSELLGTSDR & 0.97 \\
\hline HABP2_FCEIGSDDCYVGDGYSYR & 0 \\
\hline HBA_VGAHAGEYGAEALER & 0 \\
\hline HEM3_ELEHALEK & 0 \\
\hline HEMO_SGAQATWTELPWPHEK & 0 \\
\hline
\end{tabular}




\begin{tabular}{|c|c|}
\hline HEP2_TLEAQLTPR & 0 \\
\hline HGFA_LEACESLTR & 0.22 \\
\hline HPT_VGYVSGWGR & 0 \\
\hline HPTR_VVLHPNYHQVDIGLIK & 0 \\
\hline HRG_QIGSVYR & 0 \\
\hline IBP2_LIQGAPTIR & 0 \\
\hline IBP3_YGQPLPGYTTK & 0 \\
\hline IBP5_GVCLNEK & 0 \\
\hline IC1_LLDSLPSDTR & 0 \\
\hline ICAM1_VELAPLPSWQPVGK & 0 \\
\hline IDS_QSTEQAIQLLEK & 0 \\
\hline IGF2_GIVEECCFR & 0 \\
\hline IGHG1_TPEVTCVVVDVSHEDPEVK & 0 \\
\hline IGHG3_SCDTPPPCPR & 0 \\
\hline IGHM_QVGSGVTTDQVQAEAK & 1 \\
\hline IGKC_DSTYSLSSTLTLSK & 0 \\
\hline IL5_ETLALLSTHR & 0 \\
\hline INSR_VCHLLEGEK & 0.47 \\
\hline IPSP_AAAATGTIFTFR & 0 \\
\hline ITA2B_IVLLDVPVR & 0 \\
\hline ITIH1_GSLVQASEANLQAAQDFVR & 0 \\
\hline ITIH2_IYLQPGR & 0.96 \\
\hline ITIH3_EVSFDVELPK & 0.22 \\
\hline ITIH4_NVVFVIDK & 0.03 \\
\hline JAK2_SDNIIFQFTK & 0 \\
\hline K1C9_TLLDIDNTR & 0 \\
\hline K2C1_SLVNLGGSK & 0 \\
\hline K2C5_ISISTSGGSFR & 0 \\
\hline KAIN_GFQHLLHTLNLPGHGLETR & 0.22 \\
\hline KCRM_ELFDPIISDR & 0 \\
\hline KI21B_AQEQGVAGPEFK & 0 \\
\hline KLKB1_LVGITSWGEGCAR & 0 \\
\hline KNG1_QVVAGLNFR & 0 \\
\hline LA_IGCLLK & 0 \\
\hline LAMP1_ALQATVGNSYK & 0 \\
\hline LAMP2_IPLNDLFR & 0 \\
\hline LBP_GLQYAAQEGLLALQSELLR & 0 \\
\hline LCAT_SSGLVSNAPGVQIR & 0 \\
\hline LDHB_SADTLWDIQK & 0.14 \\
\hline LUM_LPSGLPVSLLTLYLDNNK & 0 \\
\hline LYAM1_AEIEYLEK & 0 \\
\hline LYRIC_WNSVSPASAGK & 0 \\
\hline LYSC_STDYGIFQINSR & 0 \\
\hline MBL2_WLTFSLGK & 0 \\
\hline
\end{tabular}




\begin{tabular}{|c|c|}
\hline MCAT_EGITGLYR & 0 \\
\hline MIA_GQVVYVFSK & 0 \\
\hline MSH2_DIYQDLNR & 0 \\
\hline NPC2_SEYPSIK & 0 \\
\hline PAFA_ASLAFLQK & 0.12 \\
\hline PEDF_ALYYDLISSPDIHGTYK & 0 \\
\hline PERM_VVLEGGIDPILR & 0 \\
\hline PGFRB_LPGFHGLR & 0 \\
\hline PGRP2_EFTEAFLGCPAIHPR & 0 \\
\hline PHLD_TLLLVGSPTWK & 0 \\
\hline PI16_WDEELAAFAK & 0 \\
\hline PLF4_ICLDLQAPLYK & 1 \\
\hline PLMN_YEFLNGR & 0 \\
\hline PLTP_AVEPQLQEEER & 0.03 \\
\hline PON1_IHVYEK & 0 \\
\hline PRDX2_TDEGIAYR & 0 \\
\hline PROC_GDSPWQVVLLDSK & 0.47 \\
\hline PROF1_STGGAPTFNVTVTK & 0 \\
\hline PROS_VYFAGFPR & 0 \\
\hline PZP_HQDGSYSTFGER & 0 \\
\hline RENI_LFDASDSSSYK & 0 \\
\hline RET4_QEELCLAR & 0 \\
\hline S10A9_LGHPDTLNQGEFK & 0 \\
\hline SAA1_FFGHGAEDSLADQAANEWGR & 0 \\
\hline SAMP_IVLGQEQDSYGGK & 0 \\
\hline SHAN3_FEDHEIEGAHLPALTK & 0 \\
\hline SHBG_TSSSFEVR & 0 \\
\hline SPP24_DYYVSTAVCR & 0 \\
\hline TENX_LQGLIPGAR & 0 \\
\hline TETN_CFLAFTQTK & 0 \\
\hline THBG_NALALFVLPK & 0 \\
\hline THRB_VTGWGNLK & 0 \\
\hline TNR5_YCDPNLGLR & 0 \\
\hline TRFE_CSTSSLLEACTFR & 0.23 \\
\hline TRFL_CSTSPLLEACEFLR & 0 \\
\hline TTHY_AADDTWEPFASGK & 0 \\
\hline UROM_VLNLGPITR & 0 \\
\hline URP2_VVLAGGVAPALFR & 0 \\
\hline VASN_LAGLGLQQLDEGLFSR & 0 \\
\hline VGFR3_SGVDLADSNQK & 0 \\
\hline VINC_QVATALQNLQTK & 0 \\
\hline VTDB_YTFELSR & 0 \\
\hline VTNC_CTEGFNVDK & 0 \\
\hline VWF_VTVFPIGIGDR & 0 \\
\hline
\end{tabular}


ZA2G_CLAYDFYPGK 0

${ }^{\text {a }}$ The proportion of feature selected for the 210 candidate features are listed. The 9 selected features chosen for the developed model are shown in bold. Protein_peptide sequence is listed for each feature. 


\section{Supplementary Table S4. Summary of unique models originating from combinations of selected features ${ }^{\mathrm{a}}$}

\begin{tabular}{|c|c|c|c|c|}
\hline Model \# & Unique model (combination of selected features) & $\begin{array}{l}\text { Number of } \\
\text { features } \\
\text { combined }\end{array}$ & $\begin{array}{c}\text { Model } \\
\text { Frequency }\end{array}$ & $\begin{array}{c}\text { Model } \\
\text { probability }\end{array}$ \\
\hline Model 1 & $\begin{array}{l}\text { ALDOA_ALQASALK + AMPN_AQIINDAFNLASAHK + C1QB_LEQGENVFLQATDK + } \\
\text { COAA1_GTHVWVGLYK + DSG3_LAEISLGVDGEGK + FA10_QEDACQGDSGGPHVTR + } \\
\text { FA9_NCELDVTCNIK + FBLN1_CVDVDECAPPAEPCGK+FCGBP_AIGYATAADCGR + } \\
\text { FHR3_AQTTVTCTEK + GPX3_NSCPPTSELLGTSDR + HGFA_LEACESLTR + } \\
\text { IGHM_QVGSGVTTDQVQAEAK + INSR_VCHLLEGEK + ITIH2_IYLQPGR + ITIH3_EVSFDVELPK + } \\
\text { KAIN_GFQHLLHTLNLPGHGLETR + PAFA_ASLAFLQK + PLF4_ICLDLQAPLYK + } \\
\text { PROC_GDSPWQVVLLSK + TRFE_CSTSSLLEACTFR + (intercept) }\end{array}$ & 21 & 9 & 0.0010 \\
\hline Model 2 & $\begin{array}{l}\text { ALDOA_ALQASALK + C1QB_LEQGENVFLQATDK + DSG3_LAEISLGVDGEGK + } \\
\text { FA9_NCELDVTCNIK + FBLN1_CVDVDECAPPAEPCGK + FCGBP_AIGYATAADCGR + } \\
\text { FHR3_AQTTVTCTEK + GPX3_NSCPPTSELLGTSDR + IGHM_QVGSGVTTDQVQAEAK + } \\
\text { ITIH2_IYLQPGR + PLF4_ICLDLQAPLYK + (intercept) }\end{array}$ & 11 & 40 & 0.7018 \\
\hline Model 3 & $\begin{array}{l}\text { ALDOA_ALQASALK + C1QB_LEQGENVFLQATDK + DSG3_LAEISLGVDGEGK + } \\
\text { FA10_QEDACQGDSGGPHVTR + FA9_NCELDVTCNIK + FBLN1_CVDVDECAPPAEPCGK } \\
\text { FCGBP_AIGYATAADCGR + FHR3_AQTTVTCTEK + GPX3_NSCPPTSELLGTSDR + } \\
\text { IGHM_QVGSGVTTDQVQAEAK + INSR_VCHLLEGEK + ITIH2_IYLQPGR + PLF4_ICLDLQAPLYK + } \\
\text { PROC_GDSPWQVVLLDSK + (intercept) }\end{array}$ & 14 & 14 & 0.1229 \\
\hline Model 4 & $\begin{array}{l}\text { ALDOA_ALQASALK + AMPN_AQIINDAFNLASAHK + C1QB_LEQGENVFLQATDK + } \\
\text { CERU_EYTDASFTNR + COAA1_GTHVWVGLYK +DSG3_LAEISLGVDGEGK + } \\
\text { FA10_QEDACQGDSGGPHVTR + FA9_NCELDVTCNIK + FBLN1_CVDVDECAPPAEPCGK + } \\
\text { FCGBP_AIGYATAADCGR + FHR3_AQTTVTCTEK + GPX3_NSCPPTSELLGTSDR + } \\
\text { HGFA_LEACESLTR + IGHM_QVGSGVTTDQVQAK + INSR_VCHLLEGEK + ITIH2_IYLQPGR + } \\
\text { ITIH3_EVSFDVELPK + } \\
\text { ITIH4_NVVFVIDK + KAIN_GFQHLLHTLNLPGHGLETR + LDHB_SADTLWDIQK + PAFA_ASLAFLQK + } \\
\text { PLF__ICLDLQAPLYK + PLTP_AVEPQLQEEER + } \\
\text { PROC_GDSPWQVLLDSK + TRFE_CSTSSLLEACTFR + (intercept) }\end{array}$ & 25 & 3 & 8.13E-06 \\
\hline
\end{tabular}




\begin{tabular}{|c|c|c|c|c|}
\hline Model 5 & $\begin{array}{l}\text { C1QB_LEQGENVFLQATDK + DSG3_LAEISLGVDGEGK + FBLN1_CVDVDECAPPAEPCGK + } \\
\text { FCGBP_AIGYATAADCGR + FHR3_AQTTVTCTEK + GPX3_NSCPPTSELLGTSDR + } \\
\text { IGHM_QVGSGVTTDQVQAEAK + ITIH2_IYLQPGR + PLF4_ICLDLQAPLYK + (intercept) }\end{array}$ & 10 & 9 & 0.0761 \\
\hline Model 6 & $\begin{array}{l}\text { ALDOA_ALQASALK + C1QB_LEQGENVFLQATDK + DSG3_LAEISLGVDGEGK + } \\
\text { FA9_NCELDVTCNIK + FBLN1_CVDVDECAPPAEPCGK + FCGBP_AIGYATAADCGR + } \\
\text { FHR3_AQTTVTCTEK + GPX3_NSCPPTSELLGTSRR + IGHM_QVGSGVTTDQVQAEAK + } \\
\text { INSR_VCHLLEGEK + ITIH2_IYLQPGR + PLF4_ICLDLQAPLYK + PROC_GDSPWQVVLLDSK + } \\
\text { (intercept) }\end{array}$ & 13 & 10 & 0.0885 \\
\hline Model 7 & $\begin{array}{l}\text { C1QB_LEQGENVFLQATDK + CXCL7_NIQSLEVIGK + DSG3_LAEISLGVDGEGK + } \\
\text { FBLN1_CVDVDECAPPAEPCGK + FCGBP_AIGYATAADCGR + FHR3_AQTTVTCTEK + } \\
\text { IGHM_QVGSGVTTDQVQAEAK + PLF4_ICLDLQAPLYK + (intercept) }\end{array}$ & 8 & 3 & 0.0043 \\
\hline Model 8 & $\begin{array}{l}\text { ALDOA_ALQASALK + C1QB_LEQGENVFLQATDK + DSG3_LAEISLGVDGEGK + } \\
\text { FA10_QEDACQGDSGGPHVTR + FA9_NCELDVTCNIK + FBLN1_CVDVDECAPPAEPCGK + } \\
\text { FCGBP_AIGYATAADCGR + FHR3_AQTTVTCTEK + GPX3_NSCPPTSELLGTSDR + } \\
\text { IGHM_QVGSGVTTDQVQAEAK + INSR_VCHLLEGEK + ITIH2_IYLQPGR + LDHB_SADTLWDIQK + } \\
\text { PLF4_ICLDLQAPLYK + PROC_GDSPWQVVLLDSK + TRFE_CSTSSLLEACTFR + (intercept) }\end{array}$ & 16 & 1 & 0.0024 \\
\hline Model 9 & $\begin{array}{l}\text { ALDOA_ALQASALK + C1QB_LEQGENVFLQATDK + COAA1_GTHVWVGLYK + } \\
\text { DSG3_LAEISLGVDGEGK + FA10_QEDACQGDSGPHVTR_+ } \\
\text { FA9_NCELDVTCNIK + FBLN1_CVDVECAPPAEPCGK + FCGBP_AIGYATAADCGR + } \\
\text { FHR3_AQTTVTCTEK + GPX3_NSCPPTSELLGTSDR + HGFA_LEACESLTR + } \\
\text { IGHM_QVGSGVTTDQVAEAK + INSR_VCHLLEGK + ITIH2_IYLQPGR + ITIH3_EVSFDVELPK + } \\
\text { KAIN_GFQHLLHTLNLPGHGLETR + LDHB_SADTLWDIQK + } \\
\text { PLF4_ICLDLQAPLYK + PROC_GDSPWQVVLLDSK + TRFE_CSTSSLLEACTFR + (intercept) }\end{array}$ & 20 & 10 & 4.56E-04 \\
\hline Model 10 & $\begin{array}{l}\text { C1QB_LEQGENVFLQATDK + CXCL7_NIQSLEVIGK + DSG3_LAEISLGVDGEGK + } \\
\text { FBLN1_CVDVDECAPPAEPCGK + FCGBP_AIGYATAADCGR + FHR3_AQTTVTCTEK + } \\
\text { GPX3_NSCPPTSELLGTSDR + IGHM_QVGSGVTTDQVQAEAK + PLF4_ICLDLQAPLYK + (intercept) }\end{array}$ & 9 & 1 & 0.0025 \\
\hline
\end{tabular}

a Ten unique models were generated. Number of features, frequency, and model probability for each unique model are

listed. Protein_peptide sequence of the components is listed for each combination. 


\section{Supplementary Table S5. Differences in protein abundance of the nine selected features between MDD and BD in}

the training set ${ }^{a}$

\begin{tabular}{|c|c|c|c|c|c|}
\hline Features & Protein name & $\begin{array}{c}\text { Gene } \\
\text { names }\end{array}$ & $\begin{array}{l}\text { Fold-change } \\
\text { (MDD/BD) }\end{array}$ & $\begin{array}{l}\text { Student's t- } \\
\text { test statistics }\end{array}$ & P-value ${ }^{b}$ \\
\hline \multicolumn{6}{|l|}{ Protein_peptide sequence } \\
\hline C1QB_LEQGENVFLQATDK & Complement $\mathrm{C} 1 \mathrm{q}$ subcomponent subunit $\mathrm{B}$ & C1QB & 0.785 & 3.548 & 5.27.E-04 \\
\hline DSG3_LAEISLGVDGEGK & Desmoglein-3 & DSG3 & 1.291 & -3.909 & 1.43.E-04 \\
\hline FBLN1_CVDVDECAPPAEPCGK & Fibulin-1 & FBLN1 & 0.776 & 2.903 & 4.29.E-03 \\
\hline FCGBP_AIGYATAADCGR & IgGFc-binding protein & FCGBP & 0.837 & 2.516 & 1.30.E-02 \\
\hline FHR3_AQTTVTCTEK & Complement factor H-related protein 3 & FHR3 & 0.718 & 2.714 & 7.48.E-03 \\
\hline GPX3_NSCPPTSELLGTSDR & Glutathione peroxidase 3 & GPX3 & 1.120 & -2.487 & 1.41.E-02 \\
\hline IGHM_QVGSGVTTDQVQAEAK & Immunoglobulin heavy constant mu & IGHM & 0.415 & 2.967 & 3.53.E-03 \\
\hline ITIH2_IYLQPGR & Inter-alpha-trypsin inhibitor heavy chain $\mathrm{H} 2$ & $\mathrm{ITIH} 2$ & 1.115 & -2.640 & 9.21.E-03 \\
\hline PLF4_ICLDLQAPLYK & Platelet factor 4 & PF4 & 2.382 & -3.692 & 3.17.E-04 \\
\hline
\end{tabular}

a Abbreviations: MDD, major depressive disorder BD, bipolar disorder

${ }^{\mathrm{b}}$ Statistically significant differences across the 9 selected features were analyzed by student's $T$-test. Bold font denotes statistical difference at $P$-value $<0.05$. Protein_peptide sequence is listed for each feature 


\section{Supplementary Table S6. Differences in protein abundance of the nine selected features between MDD, BD, and}

\section{HC in the study population (90 MDD, $90 \mathrm{BD}$, and $90 \mathrm{HC})^{\mathrm{a}}$}

\begin{tabular}{|c|c|c|c|c|c|c|c|c|c|c|c|c|c|}
\hline \multirow{2}{*}{ Features } & \multirow{2}{*}{ Protein names } & \multirow{2}{*}{$\begin{array}{c}\text { Gene } \\
\text { names }\end{array}$} & \multicolumn{2}{|c|}{ MDD vs BD vs $\mathrm{HC}$} & \multicolumn{3}{|c|}{ MDD vs BD } & \multicolumn{3}{|c|}{ MDD vs HC } & \multicolumn{3}{|c|}{ BD vs $\mathrm{HC}$} \\
\hline & & & F-statistics & $\begin{array}{l}\text { P-valueb } \\
\text { (ANOVA) }\end{array}$ & $\begin{array}{l}\text { Fold- } \\
\text { change } \\
\text { (MDD/BD) }\end{array}$ & $\begin{array}{l}\text { Mean } \\
\text { differe } \\
\text { nce }\end{array}$ & $\begin{array}{l}\text { P-valueb } \\
\text { (Tukey's } \\
\text { HSD) }\end{array}$ & $\begin{array}{l}\text { Fold- } \\
\text { change } \\
\text { (MDD/HC) }\end{array}$ & $\begin{array}{l}\text { Mean } \\
\text { differe } \\
\text { nce }\end{array}$ & $\begin{array}{l}\text { P-valueb } \\
\text { (Tukey's } \\
\text { HSD) }\end{array}$ & $\begin{array}{l}\text { Fold- } \\
\text { change } \\
\text { (BD/HC) }\end{array}$ & $\begin{array}{l}\text { Mean } \\
\text { differe } \\
\text { nce }\end{array}$ & 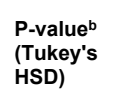 \\
\hline \multicolumn{14}{|l|}{$\begin{array}{l}\text { Protein_peptide } \\
\text { sequence }\end{array}$} \\
\hline $\begin{array}{l}\text { C1QB_LEQGENV } \\
\text { FLQATDK }\end{array}$ & $\begin{array}{l}\text { Complement } \mathrm{C} 1 \mathrm{q} \\
\text { subcomponent subunit } \mathrm{B}\end{array}$ & $\mathrm{C} 1 \mathrm{QB}$ & 5.741 & 3.62.E-03 & 0.822 & -1.965 & 2.50.E-03 & 0.886 & -1.474 & 1.12.E-02 & 1.077 & 0.792 & 3.65.E-01 \\
\hline $\begin{array}{l}\text { DSG3_LAEISLGV } \\
\text { DGEGK }\end{array}$ & Desmoglein-3 & DSG3 & 10.326 & 4.79.E-05 & 1.291 & 0.037 & 2.54.E-05 & 1.139 & 0.020 & 3.94.E-02 & 0.882 & -0.030 & 3.20.E-02 \\
\hline $\begin{array}{l}\text { FBLN1_CVDVDE } \\
\text { CAPPAAEPCGK }\end{array}$ & Fibulin-1 & FBLN1 & 7.660 & 5.82.E-04 & 0.763 & -2.881 & 5.55.E-04 & 0.816 & -2.097 & $1.72 . \mathrm{E}-02$ & 1.069 & 0.784 & 5.58.E-01 \\
\hline $\begin{array}{l}\text { FCGBP_AIGYAT } \\
\text { AADCGR }\end{array}$ & IgGFc-binding protein & FCGBP & 5.010 & 7.31.E-03 & 0.837 & -0.276 & 9.32.E-03 & 0.967 & -0.048 & 8.65.E-01 & 1.155 & 0.228 & 3.96.E-02 \\
\hline $\begin{array}{l}\text { FHR3_AQTTVTC } \\
\text { TEK }\end{array}$ & $\begin{array}{l}\text { Complement factor } \mathrm{H} \text {-related } \\
\text { protein } 3\end{array}$ & FHR3 & 3.228 & 4.22.E-02 & 0.820 & 0.231 & 3.89.E-02 & 0.978 & -0.028 & 9.79.E-01 & 1.192 & 0.238 & 2.16.E-01 \\
\hline $\begin{array}{l}\text { GPX3_NSCPPTS } \\
\text { ELLGT'TSR }\end{array}$ & Glutathione peroxidase 3 & GPX3 & 6.389 & 1.95.E-03 & 1.147 & 0.106 & 1.24.E-03 & 1.061 & 0.048 & 2.50.E-01 & 0.925 & -0.079 & 2.07.E-02 \\
\hline $\begin{array}{l}\text { IGHM_QVGSGVT } \\
\text { TDQVQAEAK }\end{array}$ & $\begin{array}{l}\text { Immunoglobulin heavy } \\
\text { constant mu }\end{array}$ & IGHM & 4.027 & 1.89.E-02 & 0.511 & -2.597 & 1.36.E-02 & 0.667 & -1.357 & 3.01.E-01 & 1.304 & 1.240 & 3.66.E-01 \\
\hline ITIH2_IYLQPGR & $\begin{array}{l}\text { Inter-alpha-trypsin inhibitor } \\
\text { heavy chain H2 }\end{array}$ & ITIH2 & 3.055 & 4.88.E-02 & 1.092 & 0.700 & 3.82.E-02 & 1.037 & 0.297 & 5.50.E-01 & 0.950 & -0.403 & 3.33.E-01 \\
\hline $\begin{array}{l}\text { PLF4_ICLDLQAP } \\
\text { LYK }\end{array}$ & Platelet factor 4 & PF4 & 27.651 & 1.22.E-11 & 2.589 & 1.171 & 2.26.E-07 & 4.619 & 1.495 & 5.14.E-09 & 1.784 & 0.324 & 2.78.E-01 \\
\hline
\end{tabular}


a Protein_peptide sequence is listed for each feature. Abbreviations: MDD, major depressive disorder; BD, bipolar disorder; HC, healthy control; ANOVA, analysis of variation; HSD, honestly significant difference

b Statistically significant differences across the 9 selected features were analyzed by ANOVA. Post hoc analysis was performed by Tukey's HSD. Bold font denotes statistical significance at $P$-value $<0.05$. 
Supplementary Table S7. Correlations between the nine selected features and demographic/clinical variables in the training set ${ }^{\mathrm{a}}$

\begin{tabular}{|c|c|c|c|c|c|c|c|c|c|c|c|c|c|c|c|c|c|c|c|}
\hline$M D D \& B D(n=144)$ & & Gender & Age & BMI & $\begin{array}{l}\text { Current } \\
\text { smoring } \\
\text { status }\end{array}$ & $\begin{array}{c}\text { Current } \\
\text { exercise } \\
\text { status }\end{array}$ & $\begin{array}{l}\text { Current } \\
\text { alcohol } \\
\text { use }\end{array}$ & $\begin{array}{c}\text { Blood } \\
\text { collection } \\
\text { time }\end{array}$ & $\begin{array}{l}\text { Fasting } \\
\text { time }\end{array}$ & $\begin{array}{c}\text { Duration } \\
\text { from } \\
\text { first } \\
\text { onset }\end{array}$ & $\begin{array}{c}\text { Duration } \\
\text { from first } \\
\text { medication }\end{array}$ & BPRS & MADRS & YMRS & HAM-A & $A P$ & MS & $A D$ & BZD/HNT \\
\hline \multicolumn{20}{|l|}{ Protein_peptide sequence } \\
\hline \multirow[t]{2}{*}{ C1QB_LEQGENVFLQATDK } & Correlation value $(r)^{b}$ & 0.139 & 0.122 & 0.035 & -0.041 & 0.083 & -0.073 & 0.057 & -0.029 & 0.073 & 0.131 & -0.036 & 0.071 & 0.071 & -0.112 & -0.011 & 0.125 & -0.007 & -0.044 \\
\hline & P-value ${ }^{c}$ & 0.097 & 0.145 & 0.678 & 0.625 & 0.324 & 0.383 & 0.497 & 0.733 & 0.387 & 0.120 & 0.668 & 0.424 & 0.395 & 0.182 & 0.896 & 0.136 & 0.933 & 0.600 \\
\hline \multirow[t]{2}{*}{ DSG3_LAEISLGVDGEGK } & Correlation value $(r)^{b}$ & 0.067 & 0.126 & -0.015 & -0.064 & 0.128 & -0.044 & 0.060 & -0.018 & -0.127 & -0.114 & -0.086 & 0.169 & -0.157 & -0.165 & -0.121 & 0.013 & 0.005 & 0.052 \\
\hline & P-value ${ }^{c}$ & 0.428 & 0.158 & 0.859 & 0.445 & 0.125 & 0.601 & 0.476 & 0.828 & 0.131 & 0.152 & 0.303 & 0.043 & 0.061 & 0.052 & 0.149 & 0.124 & 0.952 & 0.534 \\
\hline \multirow[t]{2}{*}{ FBLN1_CVDVDECAPPAEPCGK } & Correlation value $(r)^{b}$ & 0.101 & -0.047 & -0.136 & -0.033 & 0.051 & -0.071 & -0.034 & 0.100 & 0.055 & 0.047 & -0.033 & -0.122 & 0.089 & 0.103 & 0.010 & 0.042 & -0.132 & -0.031 \\
\hline & P-value ${ }^{c}$ & 0.229 & 0.576 & 0.102 & 0.699 & 0.541 & 0.401 & 0.688 & 0.232 & 0.513 & 0.573 & 0.695 & 0.253 & 0.288 & 0.164 & 0.904 & 0.619 & 0.119 & 0.712 \\
\hline \multirow[t]{2}{*}{ FCGBP_AIGYATAADCGR } & Correlation value $(r)^{b}$ & 0.065 & -0.067 & -0.138 & -0.051 & 0.102 & -0.099 & -0.007 & 0.026 & -0.028 & 0.036 & -0.016 & -0.002 & -0.084 & -0.139 & 0.035 & 0.132 & -0.157 & -0.068 \\
\hline & P-value ${ }^{c}$ & 0.441 & 0.425 & 0.100 & 0.544 & 0.223 & 0.237 & 0.936 & 0.758 & 0.740 & 0.673 & 0.852 & 0.982 & 0.317 & 0.096 & 0.681 & 0.115 & 0.060 & 0.417 \\
\hline \multirow[t]{2}{*}{ FHR3_AQTTVTCTEK } & Correlation value $(r)^{b}$ & 0.042 & 0.101 & 0.126 & 0.010 & -0.029 & -0.100 & -0.001 & -0.049 & -0.006 & 0.124 & 0.139 & -0.088 & 0.127 & -0.037 & 0.077 & 0.088 & -0.182 & 0.131 \\
\hline & P-value ${ }^{c}$ & 0.614 & 0.226 & 0.133 & 0.908 & 0.733 & 0.233 & 0.987 & 0.559 & 0.942 & 0.142 & 0.097 & 0.293 & 0.129 & 0.658 & 0.358 & 0.296 & 0.028 & 0.117 \\
\hline GPX3_NSCPPTSELLGTSDR & Correlation value $(r)^{b}$ & 0.081 & -0.101 & -0.159 & -0.035 & -0.046 & 0.063 & 0.090 & 0.110 & 0.013 & -0.107 & -0.055 & 0.110 & -0.047 & -0.154 & -0.233 & -0.092 & -0.010 & -0.047 \\
\hline
\end{tabular}




\begin{tabular}{|c|c|c|c|c|c|c|c|c|c|c|c|c|c|c|c|c|c|c|c|}
\hline & P-value ${ }^{c}$ & 0.333 & 0.226 & 0.057 & 0.674 & 0.581 & 0.455 & 0.281 & 0.188 & 0.876 & 0.204 & 0.514 & 0.188 & 0.572 & 0.049 & 0.017 & 0.353 & 0.904 & 0.574 \\
\hline \multirow[t]{2}{*}{ IGHM_QVGSGVTTDQVQAEAK } & Correlation value $(r)^{b}$ & 0.156 & -0.149 & -0.124 & -0.061 & 0.125 & -0.127 & 0.011 & 0.128 & 0.052 & 0.067 & -0.022 & -0.082 & 0.026 & -0.127 & 0.005 & 0.202 & -0.253 & -0.044 \\
\hline & P-value ${ }^{c}$ & 0.062 & 0.074 & 0.173 & 0.465 & 0.134 & 0.128 & 0.897 & 0.127 & 0.535 & 0.424 & 0.793 & 0.330 & 0.756 & 0.128 & 0.954 & 0.014 & 0.002 & 0.598 \\
\hline \multirow[t]{2}{*}{ ITIH2_IYLQPGR } & Correlation value $(r)^{b}$ & -0.006 & 0.024 & 0.056 & 0.002 & 0.081 & -0.062 & -0.013 & 0.040 & -0.124 & -0.104 & 0.041 & 0.013 & -0.053 & 0.027 & -0.136 & -0.154 & 0.163 & -0.059 \\
\hline & P-value ${ }^{c}$ & 0.946 & 0.715 & 0.502 & 0.981 & 0.337 & 0.457 & 0.875 & 0.637 & 0.138 & 0.202 & 0.622 & 0.879 & 0.524 & 0.753 & 0.081 & 0.068 & 0.050 & 0.482 \\
\hline \multirow[t]{2}{*}{ PLF4_ICLDLQAPLYK } & Correlation value $(r)^{b}$ & 0.067 & 0.092 & 0.077 & 0.044 & -0.103 & -0.004 & 0.088 & -0.148 & 0.161 & -0.043 & -0.127 & 0.128 & 0.011 & -0.013 & -0.148 & -0.125 & 0.049 & -0.146 \\
\hline & $P$-value ${ }^{c}$ & 0.428 & 0.274 & 0.360 & 0.604 & 0.219 & 0.958 & 0.296 & 0.077 & 0.054 & 0.609 & 0.124 & 0.127 & 0.872 & 0.881 & 0.220 & 0.178 & 0.561 & 0.058 \\
\hline
\end{tabular}

a Protein_peptide sequence is listed for each feature. Abbreviations: MDD, major depressive disorder; BD, bipolar disorder;

BMI, body mass index; AP, antipsychotics; MS, mood stabilizer; AD, antidepressants; BZD/HNT, benzodiazepines/hypnotics; BPRS, Brief Psychiatric Rating Scale; MADRS, Montgomery-Asberg Depression Rating Scale; YMRS, Young Mania Rating Scale; HAM-A Hamilton Anxiety Rating Scale

${ }^{\mathrm{b}}$ Correlations between the 9 selected features and continuous variables were examined based on Pearson's correlation.

Correlation values between the 9 selected features and dichotomous variables were analyzed by point-biserial correlation.

${ }^{\mathrm{c}}$ Bold font denotes statistical significance at $P$-value $<0.05$. 
Supplementary Table S8. Covariate analysis of the four features that showed statistically significant correlation with clinical variables in the training set ${ }^{\mathrm{a}}$

Features

Covariates

P-value

(ANCOVA)

Protein_peptide seqeunce

DSG3_LAEISLGVDGEGK

$\begin{array}{cc}\text { Group (MDD vs BD) } & \mathbf{0 . 0 0 4} \\ \text { MADRS } & 0.812 \\ \text { HAM-A } & 0.280\end{array}$

FHR3_AQTTVTCTEK

$\begin{array}{cc}\text { Group (MDD vs BD) } & \mathbf{0 . 0 2 5} \\ \text { AD } & 0.327\end{array}$

GPX3_NSCPPTSELLGTSDR

$\begin{array}{cc}\text { Group (MDD vs BD) } & \mathbf{0 . 0 3 1} \\ \text { AP } & 0.854 \\ \text { HAM-A } & 0.102\end{array}$

IGHM_QVGSGVTTDQVQAEAK

$\begin{array}{cc}\text { Group (MDD vs BD) } & \mathbf{0 . 0 2 6} \\ \text { MS } & 0.313 \\ \text { AD } & 0.100\end{array}$

${ }^{\text {a Protein } \_ \text {peptide sequence is listed for each feature. Abbreviations: MDD, major }}$ depressive disorder; BD, bipolar disorder; MADRS, Montgomery-Asberg S-69 
${ }^{\mathrm{b}}$ Analysis of covariance (ANCOVA) was performed to assess the potential influence of covariates that correlated significantly with the 4 features (DSG3_LAEISLGVDGEGK, FHR3_AQTTVTCTEK, GPX3_NSCPPTSELLGTSDR, and IGHM_QVGSGVTTDQVQAEAK). Bold font denotes statistical significance at $P$-value $<0.05$. 